\title{
A Proof of the Molecular Conjecture
}

\author{
Naoki Katoh • Shin-ichi Tanigawa
}

Received: 1 July 2009 / Revised: 10 June 2010 / Accepted: 6 July 2010 / Published online: 30 March 2011 (C) Springer Science+Business Media, LLC 2011

\begin{abstract}
We prove the Molecular Conjecture posed by Tay and Whiteley. This implies that a graph $G$ can be realized as an infinitesimally rigid panel-hinge framework in $\mathbb{R}^{d}$ by mapping each vertex to a rigid panel and each edge to a hinge if and only if $\left(\left(\begin{array}{c}d+1 \\ 2\end{array}\right)-1\right) G$ contains $\left(\begin{array}{c}d+1 \\ 2\end{array}\right)$ edge-disjoint spanning trees, where $\left(\left(\begin{array}{c}d+1 \\ 2\end{array}\right)-1\right) G$ is the graph obtained from $G$ by replacing each edge by $\left(\left(\begin{array}{c}d+1 \\ 2\end{array}\right)-1\right)$ parallel edges.
\end{abstract}

Keywords The molecular conjecture $\cdot$ Panel-hinge frameworks $\cdot$ Rigid realizations $\cdot$ Molecular frameworks $\cdot$ Combinatorial rigidity

\section{Introduction}

A $d$-dimensional body-hinge framework is a collection of $d$-dimensional rigid bodies connected by hinges, where a hinge is a $(d-2)$-dimensional affine subspace, i.e. pinjoints in 2-space, line-hinges in 3-space, plane-hinges in 4-space etc. The bodies are allowed to move continuously in $\mathbb{R}^{d}$ so that the relative motion of any two bodies connected by a hinge is a rotation around it (see Fig. 1) and the framework is called rigid if every such motion provides a framework isometric to the original one.

We consider a body-hinge framework as a pair $(G, \mathbf{p})$ of a multigraph $G=(V, E)$ and a mapping $\mathbf{p}$ from $e \in E$ to a $(d-2)$-dimensional affine subspace $\mathbf{p}(e)$ in $\mathbb{R}^{d}$. Namely, $v \in V$ corresponds to a body and $u v \in E$ corresponds to a hinge $\mathbf{p}(u v)$

\footnotetext{
N. Katoh

Department of Architecture and Architectural Engineering, Kyoto University, Kyoto Daigaku

Katsura, Nishikyo-ku, Kyoto 615-8540, Japan

e-mail: naoki@archi.kyoto-u.ac.jp

S. Tanigawa $(\bowtie)$

Research Institute for Mathematical Sciences, Kyoto University, Kitashirakawa-Oiwaketyo, Sakyo-ku, Kyoto, 606-8502, Japan

e-mail: tanigawa@kurims.kyoto-u.ac.jp
} 


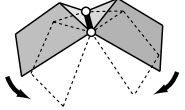

(a)

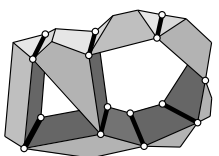

(b)

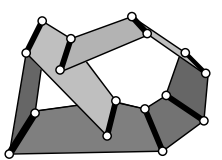

(c)

Fig. 1 (a) Two bodies connected by a hinge in three dimensions. (b) A body-hinge framework. (c) A panel-hinge framework

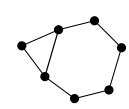

(a)

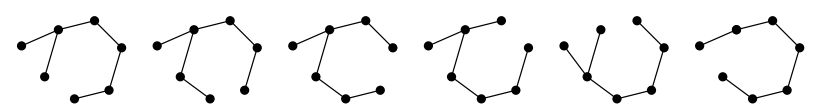

(b)

Fig. 2 (a) The underlying graph $G$ of the body-hinge framework depicted in Fig. 1(b). (b) Six edge-disjoint spanning trees in $\widetilde{G}=5 G$

which joins the two bodies corresponding to $u$ and $v$. Then, $G$ is said to be realized as a body-hinge framework $(G, \mathbf{p})$ in $\mathbb{R}^{d}$. Tay [24] and Whiteley [31] independently proved that the generic infinitesimal rigidity of a body-hinge framework is determined by the underlying graph as follows.

Proposition 1.1 [24, 31] A graph $G$ can be realized as an infinitesimally rigid bodyhinge framework in $\mathbb{R}^{d}$ if and only if $\widetilde{G}$ contains $D$ edge-disjoint spanning trees, where $D=\left(\begin{array}{c}d+1 \\ 2\end{array}\right)$ and $\tilde{G}$ denotes the graph obtained from $G$ by replacing each edge by $(D-1)$ parallel edges.

In general, the graph obtained from $G$ by replacing each edge by $k$ parallel copies is denoted by $k G$, but in this paper we shall use the simple notation $\widetilde{G}$ to denote $(D-1) G$ for our special interest in $(D-1) G$. Figure 2(a) shows the underlying graph $G$ of the body-hinge framework illustrated in Fig. 1(b). Since $5 G$ contains six edgedisjoint spanning trees as illustrated in Fig. 2(b), Tay-Whiteley's theorem ensures that $G$ can be realized as an infinitesimally rigid body-hinge framework $(G, \mathbf{p})$ in $\mathbb{R}^{3}$.

A body-hinge framework $(G, \mathbf{p})$ is called hinge-coplanar if, for each $v \in V$, all of the $(d-2)$-dimensional affine subspaces $\mathbf{p}(e)$ for edges $e$ incident to $v$ are contained in a common $(d-1)$-dimensional affine subspace (i.e. a hyperplane). In this case replacing each body by a rigid panel does not change the rigidity of the framework. Thus, a hinge-coplanar body-hinge framework is said to be a panel-hinge framework (see Fig. 1(c)). In 1984, Tay and Whiteley [25] jointly posed the following conjecture.

Conjecture 1.2 [25] Let $G=(V, E)$ be a graph. Then, $G$ can be realized as an infinitesimally rigid body-hinge framework in $\mathbb{R}^{d}$ if and only if $G$ can be realized as an infinitesimally rigid panel-hinge framework in $\mathbb{R}^{d}$.

Conjecture 1.2 is known as the Molecular Conjecture which has appeared in several different forms $[33,36]$ and has been a long standing open problem in the rigidity theory. For the special case $d=2$, Whiteley [32] proved the conjecture for a family 
of multigraphs satisfying $2|E|=3|V|-3$. Recently, the conjecture for $d=2$ was completely proved by Jackson and Jordán [14]. Their proof is done by replacing each body of a panel-hinge framework by a rigid bar-joint framework (as in [32]), and they introduced a new formula for the maximum rank of a 2-dimensional hinge-coplanar body-hinge framework ${ }^{1}$ as a 2-dimensional bar-joint framework. (The definition of bar-joint frameworks can be found in e.g. [8, 33].) Also, Jackson and Jordán [15] showed a sufficient condition of the graph to have a panel-hinge realization in higher dimension; $G$ can be realized as an infinitesimally rigid panel-hinge framework in $\mathbb{R}^{d}$ if $(d-1) G$ contains $d$ edge-disjoint spanning trees. Although the overall strategy of our proof is slightly close to that of [14], our proof directly calculates the rank of the rigidity matrix of a specific panel-hinge realization without converting to a bar-joint framework.

\subsection{Road Map of the Proof}

Our proof follows a standard strategy to prove the generic rigidity of frameworks: first we introduce two simple operations that create a larger graph from a given graph, which leads to a combinatorial induction of graphs on the number of vertices; then we prove how to geometrically implement these operations in a way that realizes a framework satisfying the desired degree of freedom. The proof is thus split into two parts; the combinatorial step and the algebraic step.

More specifically, the paper proceeds as follows. We first in Sect. 3 investigate the combinatorial property of graphs $G$ such that $\widetilde{G}$ contains $D$ edge-disjoint spanning trees. Such graphs are called body-hinge rigid graphs. More generally, $G$ is called a $k$-degree of freedom body-hinge graph (simply called a $k$-dof-graph) if it is realized as a generic body-hinge framework having $k$-degree of freedom (i.e., $k$ new edges are needed for $\widetilde{G}$ to contain $D$ edge-disjoint spanning trees by Proposition 1.1). A $k$-dof-graph is called minimal if removing any edge results in a graph that is not a $k$-dof-graph.

A combinatorial induction that generates a sequence of minimal $k$-dof-graphs is proposed in Sect. 4. For this, we introduce two operations: (i) expanding a vertex to a rigid subgraph ${ }^{2}$ and (ii) edge-splitting (i.e., subdividing an edge into two edges). In the backward direction, these correspond to (i) contraction of a rigid subgraph and (ii) splitting off at a vertex of degree two (i.e., contracting an edge incident to a vertex of degree two), respectively. The main combinatorial result states that for any 2 -edge-connected minimal $k$-dof-graph $G$, there are exactly three cases for the backward direction:

(I) if $G$ has a proper rigid subgraph, then this subgraph can be contracted so that the resulting graph $G^{\prime}$ is minimal $k$-dof-graph;

(II) if $G$ has no proper rigid subgraph with $k>0$, then there is a vertex $v$ of degree two at which splitting off produces a minimal $(k-1)$-dof-graph $G^{\prime}$;

(III) if $G$ has no proper rigid subgraph with $k=0$, then there is a vertex $v$ of degree two at which splitting off produces a minimal 0-dof-graph $G^{\prime}$.

\footnotetext{
${ }^{1}$ In [14], this is called a pin-collinear body-and-pin framework.

${ }^{2}$ This operation will be not defined explicitly, but implicitly defined as the inverse operation of contraction.
} 
In the algebraic step, we will provide a proof of the Molecular Conjecture by showing that any minimal $k$-dof-graph $G$ has a corresponding panel-hinge realization having $k$-degree of freedom (Sects. 5 and 6). Our task is to provide a geometric induction implementing the forward version of the combinatorial step according to the above three cases in a way that attains the desired degree of freedom:

- (I) can be naturally implemented as replacing a rigid panel of $G$ by a full dimensional (i.e., affinely spanning the whole space) rigid framework (Sect. 6.2).

- In case (II) we can provide a realization of $G$ from that of $G^{\prime}$ obtained by the induction (Sect. 6.3). A new panel (associated with $v$ ) is inserted so that the new infinitesimal motion caused as the result of the insertion appears only around this panel. This is essentially the same idea as that given in [33, Theorem 2.2.2] by Whiteley to prove that the so-called 1-extension preserves the infinitesimal rigidity of bar-joint frameworks.

- (III) is the most difficult case and will be discussed in Sect. 6.4. The proposed construction is basically the same as (II), but in this case we shall try $D$ distinct frameworks and claim that at least one of them is rigid. The sketch of this case will be given just before the proof.

\subsection{Connection with Molecules}

In $\mathbb{R}^{3}$ the infinitesimal rigidity of panel-hinge frameworks has a special connection with the flexibility of molecules. To identify flexible/rigid region in a protein is one of the central issues in the field of molecular biology as this could provide insight into its function and a means to predict possible changes of structural flexibility by environmental factors such as temperature and $\mathrm{pH}$. One of standard methods is to model the protein as a body-hinge or a bar-joint framework and analyze the protein's rigidity/flexibility of these mathematical models.

Consider a molecule consisting of atoms connected by covalent bonds. It is known that a molecule can be modeled as a bar-joint framework of the square of a graph (see e.g. [10, 11, 16, 35, 37]). The square of a graph $G=(V, E)$ is defined as $G^{2}=$ $\left(V, E^{2}\right)$, where $E^{2}=E \cup\{u v \in V \times V \mid u \neq v$ and $u w, w v \in E$ for some $w \in V \backslash$ $\{u, v\}\}$. A molecule can be also modeled as a body-hinge framework by regarding each atom (vertex) as a rigid body and each bond (edge) as a hinge since in the square of a graph a vertex and its neighbor form a complete graph. (A formal proof of the equivalence of the two models can be seen in [35].) Notice, however, that this bodyhinge framework has a "special" hinge configuration, i.e., all the hinges incident to a body are intersecting each other at the center of the body. Such a hinge configuration is called hinge-concurrent, and a molecule is modeled as a hinge-concurrent bodyhinge framework [25, 35-37].

Recall that taking projective dual in $\mathbb{R}^{3}$ transforms points to planes, lines to lines, and planes to points preserving their incidences. This means that the dual of a hingeconcurrent body-hinge framework is exactly a panel-hinge framework. Crapo and Whiteley [4] observed that infinitesimal rigidity is invariant under projective duality, which implies that $G$ has an infinitesimally rigid panel-hinge realization if and only if it has an infinitesimally rigid hinge-concurrent body-hinge realization. Therefore, the correctness of the Molecular Conjecture implies that the flexibility of proteins can be 
combinatorially investigated by using the well-developed tree-packing algorithm on the underlying graphs (see, e.g., [37] for more details). In fact, the so-called "pebble game" algorithm [17] for packing spanning trees is implemented in several softwares, e.g., FIRST [7, 16], ROCK [18], and others [1, 27]. From a mathematical point of view, however, the correctness proof is incomplete because they rely on the Molecular Conjecture. The result of this paper provides the theoretical validity of the algorithms behind such softwares.

\section{Preliminaries}

In this section we shall provide a formal definition of body-hinge frameworks. Refer to $[4,15,24,28,29]$ for more detailed descriptions. Throughout the paper, $d$ denotes a fixed integer with $d \geq 2$, and let $D=\left(\begin{array}{c}d+1 \\ 2\end{array}\right)$.

\subsection{Extensors}

It is known that rigidity properties are projectively invariant [4], and hence it is useful to work in $d$-dimensional projective space. To do so, for any point $p_{i}=$ $\left(p_{i, 1}, p_{i, 2}, \ldots, p_{i, d}\right) \in \mathbb{R}^{d}$, we will assign the homogeneous coordinate $\left(p_{i, 1}, p_{i, 2}\right.$, $\left.\ldots, p_{i, d}, 1\right)$, denoted by $\boldsymbol{p}_{i}$.

Let $U$ be a $(k-1)$-dimensional affine subspace of $\mathbb{R}^{d}$ determined by points $p_{1}, \ldots, p_{k}$. We denote by $A\left(\boldsymbol{p}_{1}, \ldots, \boldsymbol{p}_{k}\right)$ the $k \times(d+1)$-matrix whose $i$ th row is $\boldsymbol{p}_{i}$. For $1 \leq i_{1}<i_{2}<\cdots<i_{k} \leq d+1$, the Plücker coordinate $P_{i_{1}, i_{2}, \ldots, i_{k}}$ of $U$ is defined as the $(-1)^{1+i_{1}+i_{2}+\cdots+i_{k}}$ times the determinant of the $k \times k$-submatrix obtained from $A\left(\boldsymbol{p}_{1}, \ldots, \boldsymbol{p}_{k}\right)$ by taking $i_{j}$ th columns for $1 \leq j \leq k$. The Plücker coordinate vector of $U$ is defined as the $\left(\begin{array}{c}d+1 \\ k\end{array}\right)$-dimensional vector obtained by writing down all of possible Plücker coordinates of $U$ in some predetermined order.

Grassmann-Cayley algebra (see, e.g., [2, 6, 28]) treats a Plücker coordinate vector at a symbolic level, that is, no coordinate basis is specified, and the symbolic version of a Plücker coordinate vector is referred to as a $k$-extensor supporting $U$, which is denoted by $C(U)=\boldsymbol{p}_{1} \vee \boldsymbol{p}_{2} \vee \cdots \vee \boldsymbol{p}_{k}$. Although we will work on the coordinatized version, we would like to exploit this terminology to follow the conventional notation.

In general, for any $p_{1}, \ldots, p_{k} \in \mathbb{R}^{d}$, the k-extensor $\boldsymbol{p}_{1} \vee \boldsymbol{p}_{2} \vee \cdots \vee \boldsymbol{p}_{k}$ denotes the $\left(\begin{array}{c}d+1 \\ k\end{array}\right)$-dimensional vector consisting of $k \times k$-minors of $A\left(\boldsymbol{p}_{1}, \ldots, \boldsymbol{p}_{k}\right)$ if $k \leq d+1$ and otherwise 0 . For $P=\boldsymbol{p}_{1} \vee \cdots \vee \boldsymbol{p}_{k}$ and $Q=\boldsymbol{q}_{1} \vee \cdots \vee \boldsymbol{q}_{l}$, the join of $P$ and $Q$ is defined as $P \vee Q=\boldsymbol{p}_{1} \vee \cdots \vee \boldsymbol{p}_{k} \vee \boldsymbol{q}_{1} \vee \cdots \vee \boldsymbol{q}_{l}$.

It is known that $\boldsymbol{p}_{1} \vee \cdots \vee \boldsymbol{p}_{k} \neq \mathbf{0}$ if and only if $\left\{\boldsymbol{p}_{1}, \ldots, \boldsymbol{p}_{k}\right\}$ is linearly independent and equivalently $\left\{p_{1}, \ldots, p_{k}\right\}$ is affinely independent. Also, we formally state the following property since it will be used later.

Lemma 2.1 Let $P=\left\{p_{1}, p_{2}, \ldots, p_{d+1}\right\}$ be a set of $d+1$ points in $\mathbb{R}^{d}$ which is affinely independent. Then, the set of $(d-1)$-extensors $\left\{\boldsymbol{p}_{j_{1}} \vee \boldsymbol{p}_{j_{2}} \vee \cdots \vee \boldsymbol{p}_{j_{d-1}}: 1 \leq\right.$ $\left.j_{1}<j_{2}<\cdots<j_{d-1} \leq d+1\right\}$ is linearly independent. 
Proof Although this is a fundamental fact of extensors, let us show a proof for the completeness. Suppose that it is dependent. Then, there exist scalars $\lambda_{j_{1}, j_{2}, \ldots, j_{d-1}}$ for all $\left(\begin{array}{l}d+1 \\ d-1\end{array}\right)$ indices, indicating the dependence,

$$
\sum_{1 \leq j_{1}<\cdots<j_{d-1} \leq d+1} \lambda_{j_{1}, j_{2}, \ldots, j_{d-1}} \boldsymbol{p}_{j_{1}} \vee \boldsymbol{p}_{j_{2}} \vee \cdots \vee \boldsymbol{p}_{j_{d-1}}=\mathbf{0}
$$

and at least one scalar must be nonzero. Without loss of generality, we assume $\lambda_{1,2, \ldots, d-1} \neq 0$. Then, taking the join of (2.1) with $\boldsymbol{p}_{d} \vee \boldsymbol{p}_{d+1}$, we obtain $\lambda_{1,2, \ldots, d-1} \boldsymbol{p}_{1} \vee \cdots \vee \boldsymbol{p}_{d+1}=0$. Since $P$ is affinely independent, we also have $\boldsymbol{p}_{1} \vee \cdots \vee \boldsymbol{p}_{d+1} \neq 0$. This in turn implies $\lambda_{1,2, \ldots, d-1}=0$, which is a contradiction.

\subsection{Motions of Bodies and Hinge Constraints}

For the simplicity, let us consider 3-dimensional case for an example. A motion of a body is a direction-preserving isometry, which is written by a $4 \times 4$-matrix $M \in$ $\mathrm{SE}(3)$, an element of the special Euclidean group (see, e.g., [23]). Let us consider two bodies $B$ and $B^{\prime}$ connected by a hinge $H$ through $p_{1}=\left(p_{1, x}, p_{1, y}, p_{1, z}\right) \in \mathbb{R}^{3}$ and $p_{2}=\left(p_{2, x}, p_{2, y}, p_{2, z}\right) \in \mathbb{R}^{3}$, and suppose that $M, M^{\prime} \in \mathrm{SE}(3)$ are assigned to $B$ and $B^{\prime}$, respectively. Then, the hinge constraint by $H$ is defined as $M \boldsymbol{p}_{1}=M^{\prime} \boldsymbol{p}_{1}$ and $M \boldsymbol{p}_{2}=M^{\prime} \boldsymbol{p}_{2}$. Taking the derivative of these equalities (as $M$ and $M^{\prime}$ vary continuously), we have

$$
I \boldsymbol{p}_{i}=I^{\prime} \boldsymbol{p}_{i} \text { for } i=1,2
$$

where $I$ and $I^{\prime}$ are elements of the tangent space to the identity of SE(3), so-called the Lie algebra associated to $\mathrm{SE}(3)$, and we can regard them as infinitesimal motions assigned to $B$ and $B^{\prime}$. It is known that $I$ is represented by $\left(\begin{array}{cc}R & v^{\top} \\ 0 & 0\end{array}\right)$ by using a $3 \times 3$ skew symmetric matrix

$$
R=\left(\begin{array}{ccc}
0 & -\omega_{z} & \omega_{y} \\
\omega_{z} & 0 & -\omega_{x} \\
-\omega_{y} & \omega_{x} & 0
\end{array}\right)
$$

and $v=\left(v_{x}, v_{y}, v_{z}\right) \in \mathbb{R}^{3}$ (see, e.g., [23]). Similarly, $I^{\prime}$ is represented by $\left(\begin{array}{cc}R^{\prime} & v^{\top \top} \\ 0 & 0\end{array}\right)$. Hence, (2.2) is transformed to

$$
\begin{gathered}
\left(\begin{array}{cc}
\omega_{y}-\omega_{y}^{\prime} & \omega_{z}-\omega_{z}^{\prime} \\
p_{1, y} & p_{1, z}
\end{array}\left|+v_{x}-v_{x}^{\prime},\right| \begin{array}{cc}
\omega_{z}-\omega_{z}^{\prime} & \omega_{x}-\omega_{x}^{\prime} \\
p_{1, z} & p_{1, x}
\end{array} \mid+v_{y}-v_{y}^{\prime},\right. \\
\left|\begin{array}{cc}
\omega_{x}-\omega_{x}^{\prime} & \omega_{y}-\omega_{y}^{\prime} \\
p_{1, x} & p_{1, y}
\end{array}\right|+v_{z}-v_{z}^{\prime},\left|\begin{array}{cc}
\omega_{y}-\omega_{y}^{\prime} & \omega_{z}-\omega_{z}^{\prime} \\
p_{1, y}-p_{2, y} & p_{1, z}-p_{2, z}
\end{array}\right|, \\
\left.\left|\begin{array}{cc}
\omega_{z}-\omega_{z}^{\prime} & \omega_{x}-\omega_{x}^{\prime} \\
p_{1, z}-p_{2, z} & p_{1, x}-p_{2, x}
\end{array}\right|,\left|\begin{array}{cc}
\omega_{x}-\omega_{x}^{\prime} & \omega_{y}-\omega_{y}^{\prime} \\
p_{1, x}-p_{2, x} & p_{1, y}-p_{2, y}
\end{array}\right|\right)=0 .
\end{gathered}
$$


It is easy to observe that this equation is satisfied if and only if there exists $t \in \mathbb{R}$ such that

$$
\begin{gathered}
\left(\omega-\omega^{\prime}, v-v^{\prime}\right)=t\left(\left|\begin{array}{ll}
p_{1, x} & 1 \\
p_{2, x} & 1
\end{array}\right|,\left|\begin{array}{ll}
p_{1, y} & 1 \\
p_{2, y} & 1
\end{array}\right|,\left|\begin{array}{ll}
p_{1, z} & 1 \\
p_{2, z} & 1
\end{array}\right|,\left|\begin{array}{ll}
p_{1, y} & p_{1, z} \\
p_{2, y} & p_{2, z}
\end{array}\right|,\right. \\
\left.\left|\begin{array}{ll}
p_{1, z} & p_{1, x} \\
p_{2, z} & p_{2, x}
\end{array}\right|,\left|\begin{array}{ll}
p_{1, x} & p_{1, y} \\
p_{2, x} & p_{2, y}
\end{array}\right|\right)
\end{gathered}
$$

where $\omega=\left(\omega_{x}, \omega_{y}, \omega_{z}\right)$. This implies that, if we identify the infinitesimal motions $I$ and $I^{\prime}$ with the 6-dimensional vectors $s=(\omega, v)$ and $s^{\prime}=\left(\omega^{\prime}, v^{\prime}\right)$, the hinge $H$ constrains $s-s^{\prime}$ to be proportional to $\boldsymbol{p}_{1} \vee \boldsymbol{p}_{2}$, that is, a 2-extensor $C(H)$ supporting $H$.

This observation can be generalized to $d$-dimensional case. Suppose that two bodies $B$ and $B^{\prime}$ are connected by a hinge $A$ in $\mathbb{R}^{d}$, and elements $I$ and $I^{\prime}$ of the Lie algebra of $\operatorname{SE}(d)$ are assigned to $B$ and $B^{\prime}$. Then, we can identify $I$ and $I^{\prime}$ with $D$-dimensional vectors $s$ and $s^{\prime}$, and the hinge $H$ constrains $s$ and $s^{\prime}$ so that $s-s^{\prime}$ is in the vector space spanned by a $(d-1)$-extensor $C(H)$ supporting $H$. The $D$-dimensional vectors $s$ and $s^{\prime}$ are referred to as screw centers in e.g. [4, 15, 24, 29].

\subsection{Body-Hinge Frameworks}

A $d$-dimensional body-hinge framework $(G, \mathbf{p})$ is a pair of a multigraph $G=(V, E)$ and a mapping $\mathbf{p}$ which associates a $(d-2)$-dimensional affine subspace $\mathbf{p}(e)$ of $\mathbb{R}^{d}$ with each $e \in E$. Based on the above discussion, an infinitesimal motion of $(G, \mathbf{p})$ is defined as an assignment $S: V \rightarrow \mathbb{R}^{D}$ of a $D$-dimensional vector to each vertex such that

$$
S(u)-S(v) \text { is in the vector space spanned by a }(d-1) \text {-extensor } C(\mathbf{p}(e))
$$

for every $e=u v \in E$. An infinitesimal motion $S$ is called trivial if $S(u)=S(v)$ for all $u, v \in V$, and $(G, \mathbf{p})$ is said to be infinitesimally rigid if all infinitesimal motions of $(G, \mathbf{p})$ are trivial. We usually regard an infinitesimal motion $S$ as a point in $\mathbb{R}^{D|V|}$, which is a composition of $|V|$ vectors, $S(v) \in \mathbb{R}^{D}$ for $v \in V$, if it is clear from the context.

\subsection{Rigidity Matrix}

By the definition of infinitesimal motions, taking any basis $\left\{r_{1}(\mathbf{p}(e)), r_{2}(\mathbf{p}(e)), \ldots\right.$, $\left.r_{D-1}(\mathbf{p}(e))\right\}$ of the orthogonal complement of the vector space spanned by $C(\mathbf{p}(e)) \in$ $\mathbb{R}^{D}$, we can say that $S \in \mathbb{R}^{D|V|}$ is an infinitesimal motion of $(G, \mathbf{p})$ if and only if

$$
(S(u)-S(v)) \cdot r_{i}(\mathbf{p}(e))=0
$$

for all $i$ with $1 \leq i \leq D-1$ and for all $e=u v \in E$. Hence, the constraints (2.3) to be an infinitesimal motion are described by $(D-1)|E|$ linear equations over $S$. In other words, $S$ is infinitesimal motion of $(G, \mathbf{p})$ if and only if it is in the null space of 
a $(D-1)|E| \times D|V|$-matrix $R(G, \mathbf{p})$ written as

$$
R(G, \mathbf{p})={ }_{e=u v}\left(\begin{array}{ccccc}
\cdots & \cdots & \cdots & v & \cdots \\
\cdots \mathbf{0} \cdots & r(\mathbf{p}(e)) & \cdots \mathbf{0} \cdots & -r(\mathbf{p}(e)) & \cdots \mathbf{0} \cdots \\
\vdots & & \vdots &
\end{array}\right)
$$

where $r(\mathbf{p}(e))$ denotes a $(D-1) \times D$-matrix defined by

$$
r(\mathbf{p}(e))=\left(\begin{array}{c}
r_{1}(\mathbf{p}(e)) \\
\vdots \\
r_{D-1}(\mathbf{p}(e))
\end{array}\right) .
$$

Sequences of consecutive $(D-1)$ rows are indexed by elements of $E$, while sequences of consecutive $D$ columns are indexed by elements of $V$. We call $R(G, \mathbf{p})$ the rigidity matrix of $(G, \mathbf{p})$.

The null space of $R(G, \mathbf{p})$, which is the space of all infinitesimal motions, is denoted by $Z(G, \mathbf{p})$. Note that the dimension of $Z(G, \mathbf{p})$ is uniquely determined by $(G, \mathbf{p})$ although the entries of $R(G, \mathbf{p})$ may vary depending on the choice of basis of the orthogonal complement of the space spanned by $C(\mathbf{p}(e))$.

For $1 \leq i \leq D$, let $S_{i}^{*}$ be the infinitesimal motion of $(G, \mathbf{p})$ such that, for each $v \in V$, the $i$ th component of $S_{i}^{*}(v)$ is 1 and the others are 0 . It is not difficult to see that $S_{i}^{*}$ is contained in $Z(G, \mathbf{p})$ and $S_{i}^{*}$ is a trivial infinitesimal motion. The fact that $\left\{S_{1}^{*}, S_{2}^{*}, \ldots, S_{D}^{*}\right\}$ is linearly independent implies that the rank of $R(G, \mathbf{p})$ is at most $D|V|-D=D(|V|-1)$. Notice also that $\left\{S_{1}^{*}, \ldots, S_{D}^{*}\right\}$ spans the space of all trivial infinitesimal motions, and thus $(G, \mathbf{p})$ is infinitesimally rigid if and only if the rank of $R(G, \mathbf{p})$ is exactly $D(|V|-1)$. More generally, the dimension of the space of nontrivial infinitesimal motions is called the degree of freedom of $(G, \mathbf{p})$, which is equal to $D(|V|-1)-\operatorname{rank} R(G, \mathbf{p})$. A body-hinge framework $(G, \mathbf{p})$ is called generic if the ranks of $R(G, \mathbf{p})$ and its edge-induced submatrices take the maximum values over all realizations of $G$ [15].

\subsection{Edge-Disjoint Spanning Trees}

Let $G=(V, E)$ be a multigraph which may contain parallel edges but no selfloop. For $X \subseteq V$, let $G[X]$ be the graph induced by $X$. For $F \subseteq E$, let $V(F)$ be the set of the vertices spanned by $F$, and let $G[F]$ be the graph edge-induced by $F$, i.e., $G[F]=(V(F), F)$. For $X \subseteq V$, let $\delta_{G}(X)=\{u v \in E \mid u \in X, v \notin X\}$ and let $d_{G}(X)=\left|\delta_{G}(X)\right|$. We shall omit set brackets when describing singleton sets, e.g., $d_{G}(v)$ implies $d_{G}(\{v\})$. Throughout the paper, a partition $\mathcal{P}$ of $V$ is a collection $\left\{V_{1}, V_{2}, \ldots, V_{m}\right\}$ of vertex subsets for some positive integer $m$ such that $V_{i} \neq \varnothing$ for $1 \leq i \leq m, V_{i} \cap V_{j}=\emptyset$ for any $1 \leq i, j \leq m$ with $i \neq j$, and $\bigcup_{i=1}^{m} V_{i}=V$. Note that $\{V\}$ is a partition of $V$ for $m=1$. Let $\delta_{G}(\mathcal{P})$ and $d_{G}(\mathcal{P})$ denote the set, and the number, of edges of $G$ connecting distinct subsets of $\mathcal{P}$, respectively.

Let $\widetilde{E}$ denote the edge set of $\widetilde{G}=(D-1) G$. Also, for $e \in E$, let $\widetilde{e}$ denote the set of corresponding $D-1$ parallel copies of $e$ in $\widetilde{E}$. For $F \subseteq E$, let $\widetilde{F}=\bigcup_{e \in F} \widetilde{e}$. We index the edges of $\widetilde{e}$ by $1 \leq i \leq D-1$, and $e_{i}$, or $(e)_{i}$, denotes the $i$ th element in $\tilde{e}$. 
The result of Tay and Whiteley (Proposition 1.1) reveals the strong relation between the rigidity of body-hinge frameworks and edge-disjoint spanning trees. The following Tutte-Nash-Williams disjoint tree theorem is well-known (see e.g. [22, Chap. 51]).

Proposition 2.2 [20, 26] A multigraph $H$ contains $c$ edge-disjoint spanning trees if and only if $d_{H}(\mathcal{P}) \geq c(|\mathcal{P}|-1)$ holds for each partition $\mathcal{P}$ of $V$.

We use the following conventional notation. For a partition $\mathcal{P}$ of $V$ and a multigraph $G$, the $D$-deficiency of $\mathcal{P}$ in $\widetilde{G}$ is defined by

$$
\operatorname{def}_{\widetilde{G}}(\mathcal{P})=D(|\mathcal{P}|-1)-d_{\widetilde{G}}(\mathcal{P})=D(|\mathcal{P}|-1)-(D-1) d_{G}(\mathcal{P}),
$$

and the $D$-deficiency of $\widetilde{G}$ is defined by

$$
\operatorname{def}(\widetilde{G})=\max \left\{\operatorname{def}_{\widetilde{G}}(\mathcal{P}): \mathcal{P} \text { is a partition of } V\right\} .
$$

Note that $\operatorname{def}(\widetilde{G}) \geq 0$ since $\operatorname{def}_{\widetilde{G}}(\{V\})=0$. Proposition 2.2 implies that $\widetilde{G}$ contains $D$ edge-disjoint spanning trees if and only if $\operatorname{def}(\widetilde{G})=0$.

There is another well-known characterization of an edge set containing $D$ edgedisjoint spanning trees, which is written in terms of a matroid (see e.g. [8, 33] for the definition of a matroid in connection with rigidity theory). For $\widetilde{G}=(V, \widetilde{E})$, let us consider the matroid on $\widetilde{E}$, denoted by $\mathcal{M}(\widetilde{G})$, induced by the following nondecreasing submodular function $f: 2^{\widetilde{E}} \rightarrow \mathbb{Z}$; for any $F \subseteq \widetilde{E}, f(F)=D(|V(F)|-1)$. Namely, $F \subseteq \widetilde{E}$ is independent in $\mathcal{M}(\widetilde{G})$ if and only if $\left|F^{\prime}\right| \leq f\left(F^{\prime}\right)$ holds for every nonempty $F^{\prime} \subseteq F$ (see [21, Corollary 12.1.2]). It is known that $\widetilde{G}$ contains $D$ edge-disjoint spanning trees if and only if the rank of $\mathcal{M}(\widetilde{G})$, that is, the rank of $\widetilde{E}$ in $\mathcal{M}(\widetilde{G})$ is equal to $D(|V|-1)$. We remark that $\mathcal{M}(\widetilde{G})$ is actually the union of $D$ graphic matroids on $\widetilde{E}$, which means that an edge set is independent if and only if it can be partitioned into $D$ edge-disjoint forests.

Proposition 1.1 now implies that a multigraph $G$ can be realized as an infinitesimally rigid body-hinge framework if and only if the rank of $\mathcal{M}(\widetilde{G})$ is equal to $D(|V|-1)$. A detailed relation between the deficiency of a graph and the rank of the rigidity matrix was revealed in [15]. Let us summarize these preliminary results.

Proposition 2.3 [15] The followings are equivalent for a multigraph $G=(V, E)$ :

(i) A generic body-hinge framework $(G, \mathbf{p})$ has $k$ degree of freedom, i.e., $\operatorname{rank} R(G, \mathbf{p})=D(|V|-1)-k$.

(ii) $\operatorname{def}(\widetilde{G})=k$.

(iii) The rank of $\mathcal{M}(\widetilde{G})$ is equal to $D(|V|-1)-k$, i.e., a base of $\mathcal{M}(\widetilde{G})$ can be partitioned into $D$ edge-disjoint forests whose total cardinality is equal to $D(|V|-1)-k$.

Let us note the following relation, observed from (ii) and (iii), between the deficiency and the cardinality of a base of $\mathcal{M}(\widetilde{G})$; for a multigraph $G=(V, E)$ and a base $B$ of $\mathcal{M}(\widetilde{G})$,

$$
|B|+\operatorname{def}(\widetilde{G})=D(|V|-1) .
$$


Namely, the deficiency is equal to the corank of $\mathcal{M}(\widetilde{G})$ if $\widetilde{G}$ consists of $D(|V|-1)$ edges (see, e.g., [22] for more details).

\section{Body-Hinge Rigid Graphs}

In this section we shall further investigate combinatorial properties of body-hinge frameworks. Proposition 2.3 says that a multigraph $G=(V, E) \operatorname{satisfying} \operatorname{def}(\widetilde{G})=k$ for some integer $k$ can be realized as a generic body-hinge framework having $k$ degree of freedom. Inspired by this fact, we simply say that $G$ is a $k$-dof-graph if $\operatorname{def}(\widetilde{G})=k$ for some nonnegative integer $k$. In particular, to emphasize the relation between 0 -dof-graphs and infinitesimal rigidity given in Proposition 1.1, we sometimes refer to a 0-dof-graph as a body-hinge rigid graph. It is not difficult to see the following fact.

Lemma 3.1 Let $G$ be a body-hinge rigid graph. Then, $G$ is 2-edge-connected.

Proof Suppose that $G$ is not 2-edge-connected. Then, there exists a nonempty subset $V^{\prime}$ of $V$ satisfying $d_{G}\left(V^{\prime}\right) \leq 1$ and $V \backslash V^{\prime} \neq \emptyset$. Consider a partition $\mathcal{P}=$ $\left\{V^{\prime}, V \backslash V^{\prime}\right\}$ of $V$. Then, we have $\operatorname{def}(\widetilde{G}) \geq D(|\mathcal{P}|-1)-(D-1) d_{G}(\mathcal{P}) \geq 1$, contradicting $\operatorname{def}(\widetilde{G})=0$.

Remark Let $b$ and $c$ be positive integers and let $q=b / c$. A multigraph $G=(V, E)$ is called $q$-strong if $c G$ contains $b$ edge-disjoint spanning trees. Such graphs were first studied by Gusfield [9], where he considered the maximum value of $q$ for $G$ to be $q$-strong. This value was later called the strength of $G$ by Cunningham [5]. Checking whether $G$ is $q$-strong or not can be solved in polynomial time, if $q$ is regarded as a constant, by explicitly constructing $c G$ and checking the existence of $b$ edge-disjoint spanning trees in it, which can be efficiently done by a forest packing algorithm (and hence it can be checked in polynomial time whether $G$ is a body-hinge rigid graph). Cunningham [5] provided a strongly polynomial time algorithm for checking whether $G$ is $q$-strong and also computing the strength of $G$. The concept of strength has been extended to a general matroid by Catlin et al. [3].

In this paper, for our particular interest in $q=\frac{D}{D-1}$, we named a $\frac{D}{D-1}$-strong graph as a body-hinge rigid graph. For the rigidity of body-hinge frameworks, Jackson and Jordán $[10,12]$ recently provided several results on the $q$-strength of multigraphs, which are basically concerned with partitions of $V$ maximizing the deficiency. They also defined a minimally $q$-strong graph (with respect to edge-inclusion) and showed that a $q$-strong subgraph contained in a minimally $q$-strong graph is also minimal, which is a special case of Lemma 3.3 given in the next subsection.

\subsection{Minimally Body-Hinge Rigid Graphs}

A minimal $k$-dof-graph is a $k$-dof-graph in which removing any edge results in a graph that is not a $k$-dof-graph. In particular, a minimal 0-dof-graph is called a minimally body-hinge rigid graph. In this section we prove several new combinatorial properties of a minimal $k$-dof-graph, which will be utilized in the proof of the Molecular Conjecture. 
Lemma 3.2 Let $G$ be a minimal $k$-dof-graph for some nonnegative integer $k$. Then, $G$ is not 3-edge-connected.

Proof Suppose, for a contradiction, that $G$ is 3-edge-connected. We shall show that the graph $G_{e}$ obtained by removing an edge $e=u v \in E$ is still a $k$-dof-graph, which contradicts the minimality of $G$.

Consider any partition $\mathcal{P}=\left\{V_{1}, V_{2}, \ldots, V_{|\mathcal{P}|}\right\}$ of $V$. If $u$ and $v$ are both in the same vertex subset of $\mathcal{P}$, then $d_{G}(\mathcal{P})=d_{G_{e}}(\mathcal{P})$, and consequently $\operatorname{def}_{\widetilde{G}}(\mathcal{P})=\operatorname{def}_{\widetilde{G}_{e}}(\mathcal{P}) \leq$ $k$ holds.

Suppose $u$ and $v$ are contained in distinct subsets of $\mathcal{P}$. Without loss of generality, we assume that $u \in V_{1}$ and $v \in V_{2}$. Since $G$ is 3-edge-connected, we have $d_{G_{e}}\left(V_{i}\right) \geq 2$ for $i=1,2$ and $d_{G_{e}}\left(V_{i}\right) \geq 3$ for $i=3, \ldots,|\mathcal{P}|$. Hence, we have $d_{G_{e}}(\mathcal{P}) \geq\left\lceil\frac{3(|\mathcal{P}|-2)+2 \cdot 2}{2}\right\rceil \geq \frac{3}{2}|\mathcal{P}|-1$, which implies,

$$
\operatorname{def}_{\widetilde{G}_{e}}(\mathcal{P}) \leq D(|\mathcal{P}|-1)-(D-1)\left(\frac{3}{2}|\mathcal{P}|-1\right)=-\frac{|\mathcal{P}|}{2}(D-3)-1 .
$$

Since $d \geq 2$ and $D \geq 3$, we obtain $\operatorname{def}_{\widetilde{G}_{e}}(\mathcal{P})<0 \leq k$. Consequently, $\operatorname{def}_{\widetilde{G}_{e}}(\mathcal{P}) \leq k$ holds for any partition $\mathcal{P}$ of $V$, implying that $G_{e}$ is a $k$-dof-graph and contradicting the minimality of $G$.

For a multigraph $G=(V, E),(2.4)$ implies that an edge $e \in E$ can be removed from $G$ without changing the deficiency of $\widetilde{G}$ if and only if there exists a base $B$ of the matroid $\mathcal{M}(\widetilde{G})$ such that $B \cap \widetilde{e}=\emptyset$. Equivalently, a graph $G=(V, E)$ is a minimal $k$-dof-graph for some nonnegative integer $k$ if and only if $B \cap \tilde{e} \neq \emptyset$ for any edge $e \in E$ and any base $B$ of $\mathcal{M}(\widetilde{G})$. From this observation, it is not difficult to see the following fact.

Lemma 3.3 Let $G=(V, E)$ be a minimal $k$-dof-graph for some nonnegative integer $k$ and let $G^{\prime}=\left(V^{\prime}, E^{\prime}\right)$ be a subgraph of $G$. Suppose $\operatorname{def}\left(\widetilde{G}^{\prime}\right)=k^{\prime}$. Then $G^{\prime}$ is a minimal $k^{\prime}$-dof-graph.

Proof Consider $\mathcal{M}\left(\widetilde{G}^{\prime}\right)$, which is the matroid $\mathcal{M}(\widetilde{G})$ restricted to $\widetilde{E}^{\prime}$. Recall that the set of bases of $\mathcal{M}\left(\widetilde{G}^{\prime}\right)$ is the set of maximal members of $\left\{B \cap \widetilde{E}^{\prime} \mid\right.$ $B$ is a base of $\mathcal{M}(\widetilde{G})\}$ (see, e.g., [21, Chap. 3, 3.1.15]). Since $B \cap \widetilde{e} \neq \emptyset$ holds for any base $B$ of $\mathcal{M}(\widetilde{G})$ and for any $e \in E$ from the minimality of $G,\left(B \cap \widetilde{E}^{\prime}\right) \cap \widetilde{e} \neq \varnothing$ holds for any base $B$ of $\mathcal{M}(\widetilde{G})$ and for any $e \in E^{\prime}$. Since any base $B^{\prime}$ of $\mathcal{M}\left(\widetilde{G}^{\prime}\right)$ can be written as $B \cap \widetilde{E}^{\prime}$ with some base $B$ of $\mathcal{M}(\widetilde{G}), B^{\prime} \cap \widetilde{e} \neq \emptyset$ holds for any $e \in E^{\prime}$. This implies that $G^{\prime}$ is minimal.

In a multigraph, a pair of edges is called a cut pair if the removal of these two edges disconnects the graph. By Lemmas 3.1 and 3.2, we see that any minimally body-hinge rigid graph $G$ has a cut pair. Using this property, we can actually show a nice combinatorial property; any 2 -edge-connected minimal $k$-dof-graph contains a vertex of degree two or three. Since this is not directly used in our proof of the Molecular Conjecture, we omit the proof. Later, we shall also present a similar property in Lemma 4.6. 


\subsection{Rigid Subgraphs}

We say that a subgraph $G^{\prime}$ of $G$ is a rigid subgraph if $G^{\prime}$ is a 0-dof-graph, i.e., $\widetilde{G}^{\prime}$ contains $D$ edge-disjoint spanning trees on the vertex set of $G^{\prime}$. In this subsection we prove the following three lemmas related to rigid subgraphs.

Lemma 3.4 Let $G=(V, E)$ be a multigraph and let $X$ be a circuit of the matroid $\mathcal{M}(\widetilde{G})$. Then, $G[V(X)]$ is a rigid subgraph of $G$. More precisely, $X-e$ can be partitioned into $D$ edge-disjoint spanning trees on $V(X)$ for any $e \in X$.

Proof A circuit $X$ is a minimal dependent set of $\mathcal{M}(\widetilde{G})$ satisfying $|X|>$ $D(|V(X)|-1)$, and $X-e$ is independent in $\mathcal{M}(\widetilde{G})$ for any $e \in X$, see, e.g., [21]. From $|X|>D(|V(X)|-1)$, we have $|X-e| \geq D(|V(X)|-1)$. On the other hand, since $X-e$ is independent, we also have $|X-e| \leq D(|V(X-e)|-1) \leq$ $D(|V(X)|-1)$. As a result, $|X-e|=D(|V(X)|-1)$ and hence $X-e$ can be partitioned into $D$ edge-disjoint spanning trees on $V(X)$.

Lemma 3.5 Let $G=(V, E)$ be a minimal $k$-dof-graph for a some nonnegative integer $k$, and let $G^{\prime}=\left(V^{\prime}, E^{\prime}\right)$ be a rigid subgraph of $G$. Then, the graph obtained from $G$ by contracting $E^{\prime}$ is a minimal $k$-dof-graph.

Proof Let $H$ be the graph obtained by contracting $E^{\prime}$. By Lemma 3.1, $G^{\prime}$ is connected and hence $V^{\prime}$ becomes a single vertex after the contraction of $E^{\prime}$. Let $v^{*}$ be this new vertex in $H$, that is, $H=\left(\left(V \backslash V^{\prime}\right) \cup\left\{v^{*}\right\}, E \backslash E^{\prime}\right)$.

Let $B \widetilde{G}^{\prime}$ be a base of $\mathcal{M}\left(\widetilde{G}^{\prime}\right)$. Then, we have $\left|B_{\widetilde{G}^{\prime}}\right|=D\left(\left|V^{\prime}\right|-1\right)$ since $G^{\prime}$ is a 0 -dof-graph. Also, there exists a base $B$ of $\mathcal{M}(\widetilde{G})$ which contains $B \widetilde{G}^{\prime}$ as its subset. Let $\left\{F_{1}, F_{2}, \ldots, F_{D}\right\}$ be a partition of $B$ into $D$ edge-disjoint forests on $V$. We claim the following:

$$
F_{i} \cap \widetilde{E}^{\prime} \text { forms a spanning tree on } V^{\prime} \text { for each } 1 \leq i \leq D .
$$

To see this, notice that $B \widetilde{G}_{\widetilde{G}^{\prime}} \subset B \cap \widetilde{E}^{\prime}$ implies $\left|B \cap \widetilde{E}^{\prime}\right| \geq\left|B \widetilde{G}^{\prime}\right|=D\left(\left|V^{\prime}\right|-1\right)$. On the other hand, since $F_{i} \cap \widetilde{E}^{\prime}$ is independent in a graphic matroid, we also have $\left|F_{i} \cap \widetilde{E}^{\prime}\right| \leq\left|V\left(F_{i} \cap \widetilde{E}^{\prime}\right)\right|-1 \leq\left|V\left(B \cap \widetilde{E}^{\prime}\right)\right|-1 \leq\left|V^{\prime}\right|-1$ for each $1 \leq i \leq D$. These imply $\left|B \cap \widetilde{E}^{\prime}\right|=\sum_{i=1}^{D}\left|F_{i} \cap \widetilde{E}^{\prime}\right| \leq D\left(\left|V^{\prime}\right|-1\right) \leq\left|B \cap \widetilde{E}^{\prime}\right|$ and the equalities hold everywhere, implying $\left|F_{i} \cap \widetilde{E}^{\prime}\right|=\left|V^{\prime}\right|-1$. Thus, (3.1) holds.

Due to (3.1), after the contraction of $\widetilde{E}^{\prime}, F_{i} \backslash \widetilde{E}^{\prime}$ does not contain a cycle in $\widetilde{H}$ and again forms a forest on $\left(V \backslash V^{\prime}\right) \cup\left\{v^{*}\right\}$. In other words, $\left\{F_{1} \backslash \widetilde{E}^{\prime}, \ldots, F_{D} \backslash \widetilde{E}^{\prime}\right\}$ is a partition of $B \backslash \widetilde{E}^{\prime}$ into $D$ edge-disjoint forests on $\left(V \backslash V^{\prime}\right) \cup\left\{v^{*}\right\}$ and hence $B \backslash \widetilde{E}^{\prime}$ is independent in $\mathcal{M}(\widetilde{H})$. Since $\left|B \backslash \widetilde{E}^{\prime}\right|=|B|-\left|B \widetilde{G}^{\prime}\right|=D(|V|-1)-k$ $-D\left(\left|V^{\prime}\right|-1\right)=D\left(\left|V \backslash V^{\prime} \cup\left\{v^{*}\right\}\right|-1\right)-k, \operatorname{def}(\tilde{H}) \leq k$ follows from (2.4).

To see $\operatorname{def}(\widetilde{H}) \geq k$, let us consider a base $B_{\widetilde{H}} \subseteq \widetilde{E} \backslash \widetilde{E}^{\prime}$ of $\mathcal{M}(\widetilde{H})$. Let $\left\{S_{1}, \ldots, S_{D}\right\}$ be a partition of $B_{\widetilde{H}}$ into $D$ edge-disjoint forests on $\left(V \backslash V^{\prime}\right) \cup\left\{v^{*}\right\}$. Also, since $G^{\prime}$ is a 0-dof-graph, a base $B \widetilde{G}^{\prime}$ of $\mathcal{M}\left(\widetilde{G}^{\prime}\right)$ can be partitioned into $D$ edge-disjoint spanning trees $\left\{T_{1}, \ldots, T_{D}\right\}$ on $V^{\prime}$. Then, it is not difficult to see that $S_{i} \cup T_{i}$ forms a forest on $V$ for each $i$, and thus $B_{\widetilde{H}} \cup B_{\widetilde{G}^{\prime}}$ is an independent set of $\mathcal{M}(\widetilde{G})$. This implies 
$\left|B_{\widetilde{H}} \cup B_{\widetilde{G}^{\prime}}\right| \leq D(|V|-1)-k$. Substituting $\left|B_{\widetilde{H}}\right|=D\left(\left|V \backslash V^{\prime} \cup\left\{v^{*}\right\}\right|-1\right)-\operatorname{def}(\widetilde{H})$ and $\left|B_{\widetilde{G}^{\prime}}\right|=D\left(\left|V^{\prime}\right|-1\right)$, we obtain $\operatorname{def}(\widetilde{H}) \geq k$.

The minimality of $H$ can be checked by the same argument: If there exists a base $B_{\widetilde{H}}^{\prime}$ of $\mathcal{M}(\widetilde{H})$ which contains no edge of $\widetilde{e}$ for some $e \in E \backslash E^{\prime}$, then $B_{\widetilde{H}}^{\prime} \cup B_{\widetilde{G}^{\prime}}$ is a base of $\mathcal{M}(\widetilde{G})$ which contains no edge of $\widetilde{e}$, contradicting the minimality of $G$.

Notice that, for every circuit $X$ of $\mathcal{M}(\widetilde{G}), V(X)$ induces a 2-edge-connected subgraph by Lemmas 3.1 and 3.4. This fact leads to the following property of a multigraph that is not 2-edge-connected.

Lemma 3.6 Let $G=(V, E)$ be a $k$-dof-graph. Let $\mathcal{P}=\left\{V_{1}, V_{2}\right\}$ be a partition of $V$, and let $G_{i}=G\left[V_{i}\right]$ for $i=1,2$. Then, we have the following:

$$
\begin{aligned}
& \text { If } d_{G}(\mathcal{P})=1, \text { then } k=\operatorname{def}\left(\widetilde{G}_{1}\right)+\operatorname{def}\left(\widetilde{G}_{2}\right)+1 . \\
& \text { If } d_{G}(\mathcal{P})=0, \text { then } k=\operatorname{def}\left(\widetilde{G}_{1}\right)+\operatorname{def}\left(\widetilde{G}_{2}\right)+D .
\end{aligned}
$$

Proof Let us consider the first case. We denote by $u v$ the edge connecting between $V_{1}$ and $V_{2}$ with $u \in V_{1}$ and $v \in V_{2}$. Let $E_{1}$ and $E_{2}$ be the edge sets of $G_{1}$ and $G_{2}$, and let $E_{3}=\{u v\}$ and $G_{3}=\left(\{u, v\}, E_{3}\right)$. Then, $\left\{E_{1}, E_{2}, E_{3}\right\}$ is a partition of $E$ and moreover there exists no circuit in $\mathcal{M}(\widetilde{G})$ which intersects more than one set among $\left\{\widetilde{E}_{1}, \widetilde{E}_{2}, \widetilde{E}_{3}\right\}$ since any circuit induces a 2-edge-connected subgraph by Lemmas 3.1 and 3.4. Thus, we can decompose the matroid as $\mathcal{M}(\widetilde{G})=\mathcal{M}\left(\widetilde{G}_{1}\right) \oplus \mathcal{M}\left(\widetilde{G}_{2}\right) \oplus$ $\mathcal{M}\left(\widetilde{G}_{3}\right)$, where $\oplus$ denotes the direct sum of matroids. Since the rank of the direct sum of matroids is the sum of the ranks of these matroids, we obtain $D(|V|-1)-k=$ $D\left(\left|V_{1}\right|-1\right)-\operatorname{def}\left(\widetilde{G}_{1}\right)+D\left(\left|V_{2}\right|-1\right)-\operatorname{def}\left(\widetilde{G}_{2}\right)+D-1$, where we used the obvious fact that the rank of $\mathcal{M}\left(\widetilde{G_{3}}\right)$ is equal to $D-1$. By $|V|=\left|V_{1}\right|+\left|V_{2}\right|$, we eventually obtain the claimed relation.

The proof for the case $d_{G}(\mathcal{P})=0$ is basically the same, and hence it is omitted.

\section{Operations for Minimal $k$-dof-graphs}

In this section we shall discuss two simple operations on a minimal $k$-dof-graph. One operation is the contraction of a proper rigid subgraph; $G^{\prime}=\left(V^{\prime}, E^{\prime}\right)$ is called a proper rigid subgraph if it is a rigid subgraph of $G$ satisfying $1<\left|V^{\prime}\right|<|V|$. We have already seen in Lemma 3.5 that the contraction of a rigid subgraph produces a smaller minimal $k$-dof-graph. Another operation is a so-called splitting off operation, whose definition will be given in the next subsection. Our goal of this section is to show Lemma 4.8 , which states that any minimal $k$-dof-graph can be always converted to a smaller minimal $k$-dof-graph or minimal $(k-1)$-dof-graph by a contraction of a proper rigid subgraph or splitting off at a vertex of degree two. We use this result in the proof of the Molecular Conjecture for applying the induction. Also, as a corollary, we obtain Theorem 4.9; any minimally body-hinge rigid graph can be constructed by a sequence of these two simple operations, which must be an interesting result in its own right. 


\subsection{Splitting off Operation at a Vertex of Degree Two}

For a vertex $v$ of a graph $G$, we denote by $N_{G}(v)$ the set of vertices adjacent to $v$ in $G$. A splitting off at $v$ is an operation which removes $v$ and then inserts new edge(s) between vertices of $N_{G}(v)$. We shall consider such an operation only at a vertex $v$ of degree two. Let $N_{G}(v)=\{a, b\}$. We denote by $G_{v}^{a b}$ the graph obtained from $G$ by removing $v$ (and the edges incident to $v$ ) and then inserting a new edge $a b$. The operation that produces $G_{v}^{a b}$ from $G$ is called a splitting off at $v$ (along $a b$ ). So, the resulting graph $G_{v}^{a b}$ is isomorphic to that obtained by contracting either $v a$ or $v b$.

The main result of this subsection is Lemma 4.3, which claims that splitting off does not increase the deficiency but may not preserve the minimality of the resulting graph. Before showing Lemma 4.3, let us first investigate the relation between independent sets of $\mathcal{M}(\widetilde{G})$ and those of $\mathcal{M}\left(\widetilde{G_{v}^{a b}}\right)$ in the following lemmas.

Lemma 4.1 Let $G=(V, E)$ be a multigraph which has a vertex $v$ of degree 2 with $N_{G}(v)=\{a, b\}$. For any independent set I of $\mathcal{M}(\widetilde{G})$, there exists an independent set $I^{\prime}$ of $\mathcal{M}\left(\widetilde{G_{v}^{a b}}\right)$ satisfying $\left|I^{\prime}\right|=|I|-D$ and $\left|\widetilde{a b} \cap I^{\prime}\right|<D-1$.

Proof Let $h=|(\tilde{v a} \cup \tilde{v b}) \cap I|$ and $\left\{F_{1}, \ldots, F_{D}\right\}$ be a partition of $I$ into $D$ edgedisjoint forests on $V$. Since $d_{G}(v)=2$, clearly $d_{F_{i}}(v) \leq 2$ holds for each $i=1, \ldots, D$ (where $d_{F_{i}}(v)$ denotes the number of edges of $F_{i}$ incident to $v$ ). Let $h^{\prime}$ be the number of forests $F_{i}$ satisfying $d_{F_{i}}(v)=2$. Note that $2 h^{\prime}+\left(D-h^{\prime}\right)=h$ and $h \leq 2(D-1)$, which imply $h^{\prime} \leq D-2$. For a forest $F_{i}$ satisfying $d_{F_{i}}(v)=1$, removing the edge incident to $v$ results in a forest on $V-v$. For a forest $F_{i}$ satisfying $d_{F_{i}}(v)=2$, removing the edges incident to $v$ and inserting an edge of $\widetilde{a b}$, we can also obtain a forest on $V-v$. We hence convert each $F_{i}$ to a forest $F_{i}^{\prime}$ on $V-v$ by the above operations such that $\left|F_{i}^{\prime}\right|=\left|F_{i}\right|-1$ for each $i$. Moreover, since the total number of edges of $\widetilde{a b}$ needed to convert $F_{i}$ to $F_{i}^{\prime}$ is equal to $h^{\prime}$, which is less than $|\widetilde{a b}|=D-1$, $F_{1}^{\prime}, \ldots, F_{D}^{\prime}$ can be taken to be edge-disjoint in $\widetilde{G_{v}^{a b}}$. Let $I^{\prime}=\bigcup_{i=1}^{D} F_{i}^{\prime}$. Then, $I^{\prime}$ is an independent set of $\mathcal{M}\left(\widetilde{G_{v}^{a b}}\right)$ satisfying the required properties.

The reverse direction of splitting off at a vertex of degree two is called edgesplitting. More formally, edge-splitting (along an edge $a b$ ) is the operation that removes an edge $a b$ and then inserts a new vertex $v$ with the two new edges $v a$ and $v b$. The following lemma supplies the converse direction of Lemma 4.1.

Lemma 4.2 Let $H=(V, E)$ be a multigraph, ab be an edge of $H$, and $H_{a b}^{v}$ be the graph obtained by edge-splitting along $a b$. Let $I^{\prime}$ be an independent set of $\mathcal{M}(\tilde{H})$ with $h^{\prime}=\left|\widetilde{a b} \cap I^{\prime}\right|$. Then, (i) if $h^{\prime}<D-1$, there exists an independent set I of $\mathcal{M}\left(\widetilde{H_{a b}^{v}}\right)$ satisfying $|I|=\left|I^{\prime}\right|+D$ and $|I \cap \widetilde{v b}|=h^{\prime}+1$, and (ii) otherwise there exists an independent set $I$ of $\mathcal{M}\left(\widetilde{H_{a b}^{v}}\right)$ satisfying $|I|=\left|I^{\prime}\right|+D-1$.

Proof Let $\left\{F_{1}^{\prime}, \ldots, F_{D}^{\prime}\right\}$ be a partition of $I^{\prime}$ into $D$ edge-disjoint forests on $V$. Without loss of generality, we assume $(a b)_{i} \in F_{i}^{\prime}$ for each $1 \leq i \leq h^{\prime}$.

If $h^{\prime}<D-1$, consider the extension of each forest as follows: $F_{i}=F_{i}^{\prime}-(a b)_{i}+$ $(v a)_{i}+(v b)_{i}$ for each $1 \leq i \leq h^{\prime}, F_{i}=F_{i}^{\prime}+(v a)_{i}$ for $h^{\prime}+1 \leq i \leq D-1$ and $F_{D}=$ 
$F_{D}^{\prime}+(v b)_{h^{\prime}+1}$ (for $i=D$ ). Then, $F_{1}, \ldots, F_{D}$ are $D$ edge-disjoint forests contained in $\widetilde{H_{a b}^{v}}$ and $\bigcup_{i=1}^{D} F_{i}$, denoted $I$, is an independent set of $\mathcal{M}\left(\widetilde{H_{a b}^{v}}\right)$. Since $\left|F_{i}\right|=\left|F_{i}^{\prime}\right|+1$, we have $|I|=\left|I^{\prime}\right|+D$ and also $|I \cap \widetilde{v b}|=h^{\prime}+1$ as required.

When $h^{\prime}=D-1$, let $F_{i}=F_{i}^{\prime}-(a b)_{i}+(v a)_{i}+(v b)_{i}$ for each $1 \leq i \leq D-1$ and $F_{D}=F_{D}^{\prime}$ (for $\left.i=D\right)$. Then, $\bigcup_{i=1}^{D} F_{i}$ is an independent set of $\mathcal{M}\left(\widetilde{H_{a b}^{v}}\right)$ with cardinality $\left|I^{\prime}\right|+D-1$.

We are now ready to discuss the deficiency of the graph obtained by splitting off.

Lemma 4.3 Let $G=(V, E)$ be a minimal $k$-dof-graph which has a vertex $v$ of degree 2 with $N_{G}(v)=\{a, b\}$. Then, (i) $G_{v}^{a b}$ is either a $k$-dof-graph or a minimal $(k-1)$ dof-graph. Moreover, (ii) $G_{v}^{a b}$ is a $k$-dof-graph if and only if there is a base $B^{\prime}$ of $\mathcal{M}\left(\widetilde{G_{v}^{a b}}\right)$ satisfying $\left|\widetilde{a b} \cap B^{\prime}\right|<D-1$.

Proof Let $B$ be a base of $\mathcal{M}(\widetilde{G})$. Then, by Lemma 4.1, there exists an independent set $I^{\prime}$ of $\mathcal{M}\left(\widetilde{G_{v}^{a b}}\right)$ satisfying $\left|I^{\prime}\right|=|B|-D$ and $\left|\widetilde{a b} \cap I^{\prime}\right|<D-1$. Since $\left|I^{\prime}\right|=$ $|B|-D=D(|V|-1)-k-D=D(|V \backslash\{v\}|-1)-k$, the rank of $\mathcal{M}\left(\widetilde{G_{v}^{a b}}\right)$ is at least $D(|V \backslash\{v\}|-1)-k$. This implies

$$
\operatorname{def}\left(\widetilde{G_{v}^{a b}}\right) \leq k
$$

by (2.4), where the equality holds if and only if $I^{\prime}$ is a base of $\mathcal{M}\left(\widetilde{G_{v}^{a b}}\right)$. Thus, $\operatorname{def}\left(\widetilde{G_{v}^{a b}}\right)=k$ holds if and only if there is a base $B^{\prime}$ of $\mathcal{M}\left(\widetilde{G_{v}^{a b}}\right)$ with $\left|\widetilde{a b} \cap B^{\prime}\right|<$ $D-1$.

Let us consider the case where every base $B^{\prime}$ of $\mathcal{M}\left(\widetilde{G_{v}^{a b}}\right)$ satisfies $\left|\widetilde{a b} \cap B^{\prime}\right|=$ $D-1$. In this case $G_{v}^{a b}$ is not a $k$-dof-graph and hence (4.1) implies

$$
\operatorname{def}\left(\widetilde{G_{v}^{a b}}\right) \leq k-1
$$

By Lemma 4.2(ii), there exists an independent set $J$ of $\mathcal{M}(\widetilde{G})$ satisfying $|J|=\left|B^{\prime}\right|+$ $D-1=D(|V|-1)-\left(\operatorname{def}\left(\widetilde{G_{v}^{a b}}\right)+1\right)$, where $\left|B^{\prime}\right|=D(|V \backslash\{v\}|-1)-\operatorname{def}\left(\widetilde{G_{v}^{a b}}\right)$. We thus obtain $k=\operatorname{def}(\widetilde{G}) \leq \operatorname{def}\left(\widetilde{G_{v}^{a b}}\right)+1$ by (2.4). Combining it with (4.2), we eventually obtain $\operatorname{def}\left(\widetilde{G_{v}^{a b}}\right)=k-1$, and $G_{v}^{a b}$ is a $(k-1)$-dof-graph.

The minimality of $G_{v}^{a b}$ can be checked by using Lemma 4.2(ii) again. Suppose that there exists an edge $e$ such that $G_{v}^{a b}-e$ is still a $(k-1)$-dof-graph. Note $e \neq a b$ since every base $B^{\prime}$ of $\mathcal{M}\left(\widetilde{G_{v}^{a b}}\right)$ satisfies $\left|\widetilde{a b} \cap B^{\prime}\right|=D-1$ now. Taking a base which contains no edge of $\widetilde{e}$ in $\mathcal{M}\left(\widetilde{G_{v}^{a b}}\right)$ and then extending it by applying Lemma 4.2(ii) we will have a base of $\mathcal{M}(\widetilde{G})$ that also contains no edge of $\widetilde{e}$, contradicting the minimality of $G$. Therefore, $G_{v}^{a b}$ is a minimal $(k-1)$-dof-graph.

Applying Lemma 4.3 to the case $k=0$, we see that, for a minimally body-hinge rigid graph $G, G_{v}^{a b}$ is always body-hinge rigid. However, as we mentioned, splitting off may not preserve the minimality of $G_{v}^{a b}$. For example, for a minimally bodyhinge rigid graph $G^{\prime}$ shown in Fig. 3(a), consider the graph $G$ obtained from $G^{\prime}$ by 


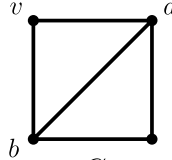

G

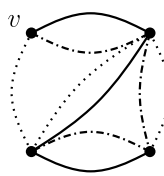

$v$
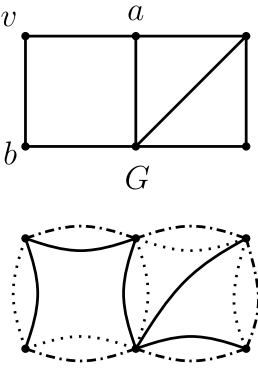
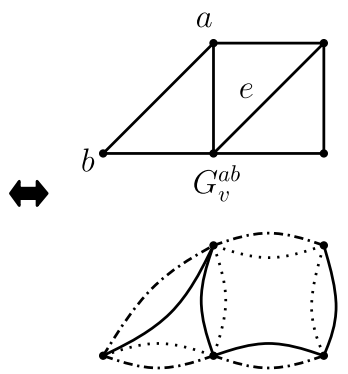

(a)
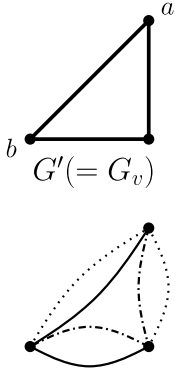

(1)

(b)

Fig. 3 (a) An example of a minimal 0-dof-graph $G$ for which $G_{v}^{a b}$ is not a minimal 0-dof-graph for $d=2$ and $D=3$. Notice that $G^{\prime}=G_{v}$ is a 0-dof-graph and hence $G_{v}^{a b}$ is not minimal. (b) An example of a minimal 0-dof-graph $G$ for which $G_{v}^{a b}$ is not minimal and also $G_{v}$ is not a 0-dof-graph for $d=2$ and $D=3$. Notice that there exist three edge-disjoint spanning trees in $\widetilde{G_{v}^{a b}}$ that contain no edge of $\widetilde{e}$

attaching a new vertex $v$ via the two new edges $v a$ and $v b$. Then, $G$ (the left one of Fig. 3(a)) is a minimally body-hinge rigid graph. On the other hand, the graph obtained from $G$ by splitting off at $v$ is not minimal while just removing $v$ (without inserting the new edge $a b$ ) from $G$ produces a minimally body-hinge rigid graph. We refer to this operation as the removal of $v$ to distinguish it from splitting off at $v$. More formally, we denote by $G_{v}$ the graph obtained by the removal of a vertex $v$ of degree two if one exists.

Lemma 4.4 Let $G=(V, E)$ be a $k$-dof-graph in which there exists a vertex $v$ of degree 2 with $N_{G}(v)=\{a, b\}$. Then, $\operatorname{def}\left(\widetilde{G_{v}}\right) \geq k$ holds. Moreover, if $\operatorname{def}\left(\widetilde{G_{v}}\right)=k$, then there exists a base $B$ of $\mathcal{M}(\widetilde{G})$ satisfying $|\widetilde{v b} \cap B|=1$.

Proof Consider a base $B^{\prime}$ of $\mathcal{M}\left(\widetilde{G_{v}}\right)$. Since $\widetilde{G_{v}}$ is a subgraph of $\widetilde{G_{v}^{a b}}, B^{\prime}$ is an independent set of $\mathcal{M}\left(\widetilde{G_{v}^{a b}}\right)$. Also, since $G$ can be obtained from $G_{v}^{a b}$ by edge-splitting along $a b$, Lemma 4.2(i) can be applied to $B^{\prime}$ with $h^{\prime}=0$ to derive that there exists an independent set $I$ of $\mathcal{M}(\widetilde{G})$ satisfying $|I|=\left|B^{\prime}\right|+D$ and $|\widetilde{v b} \cap I| \equiv 1$. This implies that $|I|=\left|B^{\prime}\right|+D=D(|V|-1)-\operatorname{def}\left(\widetilde{G_{v}}\right)$, and hence $k=\operatorname{def}(\widetilde{G}) \leq \operatorname{def}\left(\widetilde{G_{v}}\right)$ by (2.4).

If $k=\operatorname{def}(\widetilde{G})=\operatorname{def}\left(\widetilde{G_{v}}\right)$ holds, then $I$ is a base of $\mathcal{M}(\widetilde{G})$ and thus a desired base exists.

We remark that there is a situation in which $G_{v}$ is not a $k$-dof-graph and also $G_{v}^{a b}$ is not a minimal $k$-dof-graph. Figure 3(b) shows such an example.

Remarks The concept of splitting off was originated by Lovász [19], where he proved that splitting off at a vertex $v$ does not decrease the connectivity between two vertices except for $v$. Also, he proved that a graph is $2 k$-edge-connected if and only if it can be constructed from a single vertex by a sequence of two operations keeping the $2 k$-edge-connectivity: one is an edge addition and the other is the inverse of splitting off operation. Characterizing graphs having some specific property in terms of an 
inductive construction is an important topic. In particular it is known that a graph has $k$ edge-disjoint spanning trees if and only if it can be constructed from a single vertex by a sequence of three simple operations keeping the property [20]. We should remark that this result cannot be directly applied to $q$-strong graphs for a rational $q$ which is the case for our problem and there is no result concerning a construction of $q$-strong graphs to the best of our knowledge.

\subsection{Minimal $k$-dof-graphs Having No Proper Rigid Subgraph}

As shown in Fig. 3, splitting off does not preserve the minimality in general. However, if we concentrate on a graph which has no proper rigid subgraph, it can be shown that splitting off preserves the minimality. We hence concentrate on graphs having no proper rigid subgraph throughout this subsection. Let us first show properties of such graphs in Lemmas 4.5 and 4.6.

Lemma 4.5 Let $G=(V, E)$ be a multigraph which contains no proper rigid subgraph. Then, the followings hold.

(i) If $G$ is a minimal 0-dof-graph, then $(D-1)|E|<D(|V|-1)+D-1$.

(ii) If $G$ is a $k$-dof-graph with $k>0$, then $\widetilde{E}$ is the base of $\mathcal{M}(\widetilde{G})$ and hence $(D-1)|E|=D(|V|-1)-k$.

Proof (i) Let us consider the case $k=0$. Let $e$ be an arbitrary edge of $E$, and let $h^{*}$ be the minimum value of $|\widetilde{e} \cap B|$ taken over all bases $B$ of $\mathcal{M}(\widetilde{G})$. Also, let $B^{*}$ be a base of $\mathcal{M}(\widetilde{G})$ satisfying $\left|\widetilde{e} \cap B^{*}\right|=h^{*}$. Notice $h^{*} \geq 1$ due to the minimality of $G$. We shall show the following fact:

$$
\widetilde{E} \backslash \widetilde{e} \subset B^{*}
$$

Suppose, for a contradiction, that an edge $f_{i} \in \widetilde{E} \backslash \widetilde{e}$ is not contained in $B^{*}$. We consider the fundamental circuit $X$ within $B^{*}+f_{i}$. Then, $G[V(X)]$ is a rigid subgraph by Lemma 3.4. Since there exists no proper rigid subgraph in $G, V(X)=V$ must hold. Moreover, $X \cap \widetilde{e} \neq \emptyset$ also holds since otherwise there exist $D$ edge-disjoint spanning trees on $V$ which contain no edge of $\widetilde{e}$ by Lemma 3.4, contradicting the minimality of $G$. Therefore, there exists the base $B=B^{*}+f_{i}-e_{j}$ of $\mathcal{M}(\widetilde{G})$ satisfying $|B \cap \widetilde{e}|<\left|B^{*} \cap \widetilde{e}\right|=h^{*}$, where $e_{j} \in X \cap \widetilde{e}$. This contradicts the choice of $B^{*}$, and hence (4.3) follows.

By (4.3) and $\left|B^{*}\right|=D(|V|-1)$, the total number of edges in $\widetilde{G}$ is equal to $\left|B^{*}\right|+$ $\left(|\widetilde{e}|-h^{*}\right)=D(|V|-1)+\left(D-1-h^{*}\right)$, which is less than $D(|V|-1)+D-1$ by $h^{*} \geq 1$.

(ii) Let us consider the case of $k>0$. We shall show the following fact, which is analogous to (4.3):

$$
\widetilde{E} \text { is independent in } \mathcal{M}(\widetilde{G}) \text {. }
$$

Suppose for a contradiction that $\widetilde{E}$ is dependent. Then, there exists an edge $f_{i} \in \widetilde{E}$ that is not contained in a base $B$ of $\mathcal{M}(\widetilde{G})$. Consider again the fundamental circuit $X$ within $B+f_{i}$. By Lemma 3.4, $G[V(X)]$ is a rigid subgraph. Since $G$ contains 
no proper rigid subgraph from the lemma assumption, $V(X)=V$ must hold. Moreover, $V(X)=V$ implies that $G$ contains $D$ edge-disjoint spanning trees on $V$, which consist of edges of $X$. This in turn implies $k=0$, contracting $k>0$. Thus, (4.4) follows.

The following lemma shows the existence of small degree vertices.

Lemma 4.6 Let $G=(V, E)$ be a 2-edge-connected minimal $k$-dof-graph which contains no proper rigid subgraph. Then, either $G$ is a cycle graph of at most d vertices or it contains a chain $v_{0} v_{1} \ldots v_{d}$ of length $d$ such that $v_{i} v_{i+1} \in E$ for $0 \leq i \leq d-1$ and $d_{G}\left(v_{i}\right)=2$ for $1 \leq i \leq d-1$.

Proof Let us denote the average degree of the vertices of $G$ by $d_{\text {avg }}$. Lemma 4.5 implies $(D-1)|E|<D(|V|-1)+D-1<D|V|$. Hence, we have

$$
d_{\text {avg }}=\frac{2|E|}{|V|}<\frac{2 D}{D-1}=2+\frac{2}{D-1} \leq 3
$$

where the last inequality follows from $D \geq 3$. This implies that $G$ has a vertex of degree two.

If $G$ is a cycle, then the statement clearly holds. (If it consists of more than $d$ vertices, then the latter property holds.) Hence let us consider the case where $G$ contains a vertex of degree more than two. For a nonnegative integer $i$, let $X_{i}=\left\{v \in V \mid d_{G}(v)=i\right\}$. Note that, since $G$ is 2-edge-connected, $X_{0}=\emptyset$ and $X_{1}=\emptyset$ hold. We say that a chain $u_{0} u_{1} \ldots u_{j}$ is maximal if $d_{G}\left(u_{0}\right)>2, d_{G}\left(u_{j}\right)>2$ and $d_{G}\left(u_{i}\right)=2$ for all $1 \leq i \leq j-1$. Let $\mathcal{C}$ be the collection of all maximal chains in $G$. Note that $\mathcal{C}$ is nonempty (because $G$ is not a cycle), and each vertex of degree two belongs to exactly one maximal chain. Suppose, for a contradiction, that the length of each maximal chain is at most $d-1$. Then, each maximal chain contains at most $d-2$ vertices of degree two and hence we have

$$
\left|X_{2}\right| \leq(d-2)|\mathcal{C}| \text {. }
$$

For a maximal chain $u_{1} u_{2} \ldots u_{j}$, we call the edges $u_{1} u_{2}$ and $u_{j-1} u_{j}$ the end edges of the chain. Then the set of all end edges of the maximal chains in $\mathcal{C}$ is a subset of the edges incident to the vertices of $\bigcup_{i \geq 3} X_{i}$. Hence we have

$$
2|\mathcal{C}| \leq \sum_{i \geq 3} i\left|X_{i}\right|
$$

Combining (4.6) and (4.7), we obtain

$$
2\left|X_{2}\right| \leq \sum_{i \geq 3} i(d-2)\left|X_{i}\right| .
$$

Summing up this inequality and (the twice of) $|V|=\sum_{i \geq 2}\left|X_{i}\right|$, we further obtain

$$
\sum_{i \geq 3}(i(d-2)+2)\left|X_{i}\right| \geq 2|V|
$$


It is not difficult to see that the following inequality holds:

$$
(D-1)(i-2) \geq i(d-2)+2 \text { for all } i \geq 3 \text {. }
$$

Hence, by (4.8), we have

$$
\sum_{i \geq 3}(D-1)(i-2)\left|X_{i}\right| \geq 2|V|
$$

As a result,

$$
\begin{aligned}
d_{\mathrm{avg}} & =\frac{\sum_{i \geq 2} i\left|X_{i}\right|}{|V|} \\
& =2+\frac{\sum_{i \geq 3}(i-2)\left|X_{i}\right|}{|V|} \quad\left(\text { by } 2|V|=\sum_{i \geq 2} 2\left|X_{i}\right|\right) \\
& =2+\frac{\sum_{i \geq 3}(D-1)(i-2)\left|X_{i}\right|}{(D-1)|V|} \\
& \geq 2+\frac{2}{D-1} \quad(\text { by (4.9)) } \\
& >d_{\text {avg }} \quad(\text { by (4.5)) }
\end{aligned}
$$

which is a contradiction.

Let us start to investigate the deficiencies of graphs obtained by the operations defined in Sect. 4.1, assuming that $G$ contains no proper rigid subgraph.

Lemma 4.7 Let $G=(V, E)$ be a minimal $k$-dof-graph with $|V| \geq 3$ which contains no proper rigid subgraph. Let $v$ be a vertex of degree two. Then, $\operatorname{def}\left(\widetilde{G_{v}}\right)>k$.

Proof Note that $G_{v}$ is a proper subgraph of $G$. Since there exists no proper rigid subgraph in $G, G_{\mathcal{V}}$ is not a 0 -dof-graph. This proves the statement for $k=0$.

When $k>0, \widetilde{E}$ is the base of $\mathcal{M}(\widetilde{G})$ from Lemma $4.5($ ii). Since $\widetilde{E}$ is the unique base of $\mathcal{M}(\widetilde{G})$ and $|\widetilde{v b} \cap \widetilde{E}| \neq 1$ holds, Lemma $4.4 \operatorname{implies} \operatorname{def}\left(\widetilde{G_{v}}\right)>k$.

Lemma 4.8 Let $G=(V, E)$ be a minimal $k$-dof-graph that contains no proper rigid subgraph. Then, for any vertex $v$ of degree two with $N_{G}(v)=\{a, b\}$, the following holds:

(i) If $k=0$, then $G_{v}^{a b}$ is a minimal 0-dof-graph.

(ii) If $k>0$, then $G_{v}^{a b}$ is a minimal $(k-1)$-dof-graph.

Proof We remark that $G_{v}$ is not a $k$-dof-graph by Lemma 4.7. Also, by Lemma 4.3, $G_{v}^{a b}$ is either a $k$-dof-graph or a minimal $(k-1)$-dof-graph.

Let us show (i). In the case $k=0, G_{v}^{a b}$ is clearly a 0 -dof-graph and thus we only need to show the minimality of $G_{v}^{a b}$. To see this, we claim the following:

For any circuit $X$ of the matroid $\mathcal{M}\left(\widetilde{G_{v}^{a b}}\right), X \cap \widetilde{a b} \neq \varnothing$ holds. 
Suppose $X \cap \widetilde{a b}=\emptyset$. Then, $X$ is a subset of $\widetilde{E}$. Hence, $X$ is a circuit of $\mathcal{M}(\widetilde{G})$ with $v \notin V(X)$. Lemma 3.4 implies that $G[V(X)]$ is a proper rigid subgraph of $G$, which contradicts the lemma assumption. Thus (4.10) follows.

Suppose that $G_{v}^{a b}$ is not a minimal 0-dof-graph. Then, there exists an edge $e$ such that $G_{v}^{a b}-e$ is still a 0 -dof-graph. (Note $e \neq a b$ since $G_{v}$ is not a 0-dof-graph by Lemma 4.7.) Also, there exists a base $B_{1}$ of $\mathcal{M}\left(\widetilde{G_{v}^{a b}}\right)$ with $B_{1} \cap \widetilde{e}=\emptyset$. Let $h=$ $\left|B_{1} \cap \widetilde{a b}\right|$, and let us denote the edges of $B_{1} \cap \widetilde{a b}$ by $(a b)_{1}, \ldots,(a b)_{h}$. We repeatedly perform the following process for $i=1, \ldots, h$ : Insert an edge $e_{i} \in \widetilde{e}$ into $B_{i}$ and let $X_{i}$ be the fundamental circuit of $B_{i}+e_{i}$ (in $\mathcal{M}\left(\widetilde{G_{v}^{a b}}\right)$ ). By (4.10), $X_{i} \cap \widetilde{a b} \neq \emptyset$ holds and hence we can obtain a new base $B_{i+1}$ by removing $(a b)_{i} \in X_{i} \cap \widetilde{a b}$. (Namely, we obtain the base $B_{i+1}=B_{i}+e_{i}-(a b)_{i}$ of $\mathcal{M}\left(\widetilde{G_{v}^{a b}}\right)$.) Repeating this process, we eventually obtain the base $B_{h+1}$ of $\mathcal{M}\left(\widetilde{G_{v}^{a b}}\right)$, which contains no edge of $\widetilde{a b}$. Note that $B_{h+1}$ is a base of $\mathcal{M}\left(\widetilde{G_{v}}\right)$ as well as a base of $\mathcal{M}\left(\widetilde{G_{v}^{a b}}\right)$ with cardinality $\left|B_{h+1}\right|=$ $\left|B_{1}\right|=D(|V \backslash\{v\}|-1)$, which implies $\operatorname{def}\left(\widetilde{G_{v}}\right)=0$ by (2.4). This contradicts that $G_{v}$ is not a 0-dof-graph (by Lemma 4.7) and thus (i) follows.

Next let us show (ii). If $G_{v}^{a b}$ is not a $k$-dof-graph, then the statement follows because $G_{v}^{a b}$ is a minimal $(k-1)$-dof-graph by Lemma 4.3. Suppose, for a contradiction, that $G_{\mathcal{L}}^{a b}$ is a $k$-dof-graph. By Lemma 4.3 there exists a base $B^{\prime}$ of $\mathcal{M}\left(\widetilde{G_{v}^{a b}}\right)$ satisfying $\left|a b \cap B^{\prime}\right|<D-1$. Without loss of generality, we assume $(a b)_{1} \notin B^{\prime}$. Consider the fundamental circuit $Y$ of $B^{\prime}+(a b)_{1}$ and let $G^{\prime}=G[V(Y)]$. Then, by Lemma 3.4, $G^{\prime}$ is a 0-dof-graph on $V(Y)$. Since $G_{v}^{a b}$ is a $k$-dof-graph with $k>0$, $G^{\prime}$ must be a proper subgraph of $G_{v}^{a b}$, i.e., $V(Y)$ is a proper subset of $V \backslash\{v\}$. Let $I=Y-(a b)_{1}$. Observe that $I$ is an independent set of $\mathcal{M}\left(\widetilde{G}^{\prime}\right)$ with $|I \cap \widetilde{a b}|<D-1$ due to $(a b)_{1} \notin I \subseteq B^{\prime}$. We perform edge-splitting along $a b$ in $G^{\prime}$. Lemma 4.2(i) implies that the resulting graph contains an independent set with cardinality $|I|+D$, which is equal to $D(|V(Y)|-1)+D=D(|V(Y) \cup\{v\}|-1)$. Hence the resulting graph is a 0-dof-graph. Moreover, this 0-dof-graph is a proper subgraph of $G$ since $V(Y)$ is a proper subset of $V \backslash\{v\}$. Therefore, $G$ contains a proper rigid subgraph, which contradicts the lemma assumption.

\subsection{A Sequence of Minimally Body-Hinge Rigid Graphs}

Combining the results obtained so far, it is not difficult to observe the existence of an inductive sequence of minimally body-hinge rigid graphs.

Theorem 4.9 Let $G$ be a minimally body-hinge rigid graph with $|V| \geq 2$. Then, there exists a sequence $G=G_{1}, G_{2}, \ldots, G_{m}$ of minimally body-hinge rigid graphs such that

- $G_{m}$ is a graph consisting of two vertices $\{u, v\}$ and two parallel edges connecting $u$ and $v$, and

- $G_{i+1}$ is obtained from $G_{i}$ by either splitting off at a vertex of degree 2 or the contraction of a proper rigid subgraph for each $i=1, \ldots, m-1$.

Proof By Lemma 3.1, any minimally body-hinge rigid graph is a 2-edge-connected 0 -dof-graph. Hence, if $G$ contains no proper rigid subgraph, then $G$ has a vertex of 
degree two by Lemma 4.6. Combining this fact with Lemma 4.8, either (i) $G$ contains a proper rigid subgraph $G^{\prime}=\left(V^{\prime}, E^{\prime}\right)$ or (ii) there exists a vertex $v$ of degree 2 with $N_{G}(v)=\{a, b\}$ such that $G_{v}^{a b}$ is a minimal 0-dof-graph, that is, a minimally bodyhinge rigid graph. Recall that, if (i) holds, the graph obtained by the contraction of $E^{\prime}$ is again a minimally body-hinge rigid graph by Lemma 3.5. Hence, in either case, we obtain a new minimally body-hinge rigid graph $G_{2}=\left(V_{2}, E_{2}\right)$ with $2 \leq\left|V_{2}\right|<$ $|V|$. By inductively repeating this process, we eventually obtain a minimally bodyhinge rigid graph $G_{m}$ which consists of two vertices and two parallel edges between them. This inductive process shows a desired sequence of minimally body-hinge rigid graphs.

\section{Infinitesimally Rigid Panel-Hinge Realizations}

Recall the notation given in Sect. 2.4: $C(\mathbf{p}(e))$ denotes a $(d-1)$-extensor supporting the $(d-2)$-dimensional affine space $\mathbf{p}(e),\left\{r_{1}(\mathbf{p}(e)), \ldots, r_{D-1}(\mathbf{p}(e))\right\}$ denotes a basis of the orthogonal complement of the 1-dimensional vector space spanned by $C(\mathbf{p}(e))$, and $r(\mathbf{p}(e))$ denotes the $(D-1) \times D$-matrix whose $i$ th row vector is $r_{i}(\mathbf{p}(e))$. The rigidity matrix of $(G, \mathbf{p})$ is written as $(2.4)$, where consecutive $D-1$ rows and $D$ columns are indexed by elements of $E$ and $V$, respectively. Let us denote the ( $D-$ 1) $\times D|V|$-submatrix associated with $e \in E$ by $R(G, \mathbf{p} ; e)$, i.e.,

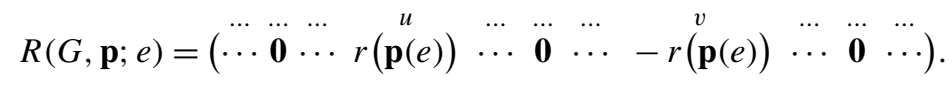

We remark $\operatorname{rank} R(G, \mathbf{p} ; e)=D-1$ since $\operatorname{rank} r(\mathbf{p}(e))=D-1$. Also, we consider the one-to-one correspondence between $e_{i} \in \widetilde{e}$ and the $i$ th row of $R(G, \mathbf{p} ; e)$, which is denoted by $R\left(G, \mathbf{p} ; e_{i}\right)$. Namely, for $e=u v \in E$ and $1 \leq i \leq D-1$, it is a $D|V|$-dimensional vector described as

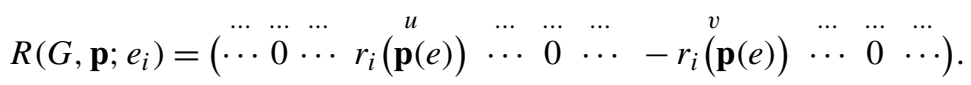

Any vector in $\mathbb{R}^{D|V|}$ can be regarded as a composition of $|V|$ vectors in $\mathbb{R}^{D}$ each of which is associated with a vertex $v \in V$ in a natural way. Similarly, let us denote by $R(G, \mathbf{p} ; v)$ the $(D-1)|E| \times D$-submatrix of $R(G, \mathbf{p})$ induced by the consecutive $D$ columns associated with $v$. For $F \subseteq E$ and $X \subseteq V, R(G, \mathbf{p} ; F, X)$ denotes the submatrix of $R(G, \mathbf{p})$ induced by the rows of $R(G, \mathbf{p} ; e)$ for $e \in F$ and the columns of $R(G, \mathbf{p} ; v)$ for $v \in X$.

We need the following technical lemma.

Lemma 5.1 Let $(G, \mathbf{p})$ be a body-hinge framework in $\mathbb{R}^{d}$ for a multigraph $G=$ $(V, E)$. Then, for any vertex $v \in V, \operatorname{rank} R(G, \mathbf{p} ; E, V \backslash\{v\})=\operatorname{rank} R(G, \mathbf{p})$ holds, i.e., the rank of the rigidity matrix is invariant under the removal of the consecutive $D$ columns associated with $v$.

Proof For $1 \leq i \leq D$, let $b_{i}$ be the vector in $\mathbb{R}^{D|V|}$ such that the $i$ th coordinate of the consecutive $D$ coordinates associated with $v$ is equal to 1 and the other entries are 
all 0 . Let $R^{\prime}$ be the matrix obtained from $R(G, \mathbf{p})$ by adding $b_{i}$ as new rows for all $i$. Then, appropriate fundamental row operations change $R^{\prime}$ to the following form:

$$
R^{\prime}=\left(\begin{array}{c|c}
v \quad V \backslash\{v\} \\
I \mid \mathbf{0} \\
\hline R(G, \mathbf{p})
\end{array}\right) \stackrel{\text { Row operations }}{\longrightarrow}\left(\begin{array}{c|c}
v & V \backslash\{v\} \\
\hline I & \mathbf{0} \\
\hline \mathbf{0} & R(G, \mathbf{p} ; E, V \backslash\{v\})
\end{array}\right)
$$

where $I$ denotes the $D \times D$ identity matrix. Hence, the statement is true if and only if $\operatorname{rank} R^{\prime}=\operatorname{rank} R(G, \mathbf{p})+D$.

It is known that the space of nontrivial infinitesimal motions is unchanged if one of bodies is fixed (see, e.g., [29, Sect. 2]). Since adding $b_{1}, \ldots, b_{D}$ means the tie down of the body associated with $v, \operatorname{rank} R^{\prime}=\operatorname{rank} R(G, \mathbf{p})+D$ holds.

\subsection{Generic Nonparallel Panel-Hinge Realizations}

A panel-hinge framework is a body-hinge framework $(G, \mathbf{p})$ such that, for each $v \in V$, all of the $(d-2)$-dimensional affine subspaces $\mathbf{p}(e)$ for $e \in \delta_{G}(v)$ are contained in a common hyperplane. We denote this hyperplane for each $v \in V$ by $\Pi_{G, \mathbf{p}}(v)$. (If the choice of such a hyperplane is not unique, we choose an arbitrary one.) As in body-hinge frameworks, the infinitesimal rigidity of a panel-hinge framework $(G, \mathbf{p})$ is defined in terms of the rigidity matrix $R(G, \mathbf{p})$ given in (2.4).

Before providing a proof of the Molecular Conjecture, we need to introduce the concept of nonparallel realizations, which is essentially taken from [15, Sect. 7]. For a simple graph $G$ (i.e., no parallel edges exist in $G$ ), a panel-hinge framework $(G, \mathbf{p})$ is called nonparallel ${ }^{3}$ if $\Pi_{G, \mathbf{p}}(u)$ and $\Pi_{G, \mathbf{p}}(v)$ are not parallel for any distinct $u, v \in V$; $\Pi_{G, \mathbf{p}}(u)$ and $\Pi_{G, \mathbf{p}}(v)$ are said to be nonparallel if $\Pi_{G, \mathbf{p}}(u) \cap \Pi_{G, \mathbf{p}}(v)$ is a $(d-2)$ dimensional affine subspace.

For a simple graph $G=(V, E)$, consider a mapping $\mathbf{c}: V \rightarrow \mathbb{R}^{d}$ such that $\mathbf{c}(u)$ and $\mathbf{c}(v)$ are linearly independent for each $u, v \in V$ with $u \neq v$. Then, a hyperplane associated with $v \in V$ with respect to $\mathbf{c}$ is defined as $\Pi(v)=\left\{x \in \mathbb{R}^{d} \mid x \cdot \mathbf{c}(v)+\right.$ $1=0\}$. Since $\mathbf{c}(u)$ and $\mathbf{c}(v)$ are linearly independent, $\Pi(u) \cap \Pi(v)$ is a $(d-2)$ dimensional affine space. Hence, the mapping $\mathbf{c}$ induces a mapping $\mathbf{p}$ on $E$, i.e., $\mathbf{p}(u v)=\Pi(u) \cap \Pi(v)$ for $u v \in E$, and $(G, \mathbf{p})$ is a nonparallel panel-hinge framework of $G$. Conversely, given a nonparallel $(G, \mathbf{p}), \mathbf{p}$ induces the mapping $\mathbf{c}: V \rightarrow \mathbb{R}^{d}$ such that $\Pi_{G, \mathbf{p}}(v)=\left\{x \in \mathbb{R}^{d} \mid x \cdot \mathbf{c}(v)+1=0\right\}$ for each $v \in V$ (provided that no panel passes through the origin).

A nonparallel panel-hinge framework $(G, \mathbf{p})$ is called generic if the set of coordinates of $\mathbf{c}(v)$ for all $v \in V$ is algebraically independent over the rational field. ${ }^{4} \mathrm{We}$ note that almost all nonparallel panel-hinge realizations are generic. Since each entry of $R(G, \mathbf{p})$ can be written as a polynomial of the coordinates of $\mathbf{c}(v), v \in V$ (see [15, Lemma 7.1] for more detail), the rank of $R(G, \mathbf{p})$ takes the maximum value over all nonparallel panel-hinge realizations of $G$ if $(G, \mathbf{p})$ is generic.

\footnotetext{
${ }^{3}$ In [15], such a framework is called nondegenerate.

${ }^{4}$ The definition in terms of algebraic independence produces a smaller class of frameworks than that of conventional generic frameworks (in terms of the maximality of rigidity matrices). We just use this definition to make our proof simpler.
} 
It is known that, even though $(G, \mathbf{p})$ has some parallel panels, we can perturb them so that the resulting realization becomes nonparallel without decreasing the rank of the rigidity matrix [15, Lemma 7.1]. The following lemma states a special case of this result, but let us provide a proof for completeness.

Lemma 5.2 Let $G$ be a simple graph and $(G, \mathbf{p})$ be a panel-hinge realization of $G$. Suppose that there exists a pair $(a, b) \in V \times V$ with $a \neq b$ satisfying $\Pi_{G, \mathbf{p}}(a)=$ $\Pi_{G, \mathbf{p}}(b)$ and that $\Pi_{G, \mathbf{p}}(u)$ and $\Pi_{G, \mathbf{p}}(v)$ are nonparallel for every pair $(u, v) \in V \times V$ with $u \neq v$ except for $(a, b)$. Then, there is a nonparallel panel-hinge realization $\left(G, \mathbf{p}^{\prime}\right)$ satisfying $\operatorname{rank} R\left(G, \mathbf{p}^{\prime}\right) \geq \operatorname{rank} R(G, \mathbf{p})$.

Proof We shall only consider the case of $a b \in E$. The case of $a b \notin E$ can be handled similarly. Note that $a b$ is unique because $G$ is simple.

Since the rank of the rigidity matrix is invariant under an isometric transformation of the whole framework, we can assume $\Pi_{G, \mathbf{p}}(a)=\Pi_{G, \mathbf{p}}(b)=\left\{x=\left(x_{1}, \ldots, x_{d}\right) \in\right.$ $\left.\mathbb{R}^{d} \mid x_{d}=0\right\}$ and $\mathbf{p}(a b)=\left\{x \in \mathbb{R}^{d} \mid x_{d-1}=0, x_{d}=0\right\}$. We shall rotate $\Pi_{G, \mathbf{p}}(a)$ continuously around $\mathbf{p}(a b)$. To indicate the rotation, let us introduce a parameter $t \in \mathbb{R}$ and define $\Pi^{t}(a)=\left\{x \in \mathbb{R}^{d} \mid t x_{d-1}+x_{d}=0\right\}$. Note that $\Pi^{0}(a)=\Pi_{G, \mathbf{p}}(a)$ and $\mathbf{p}(a b) \subseteq \Pi^{t}(a) \cap \Pi_{G, \mathbf{p}}(b)$ for any $t \in \mathbb{R}$.

Since $\Pi^{0}(a)$ and $\Pi_{G, \mathbf{p}}(v)$ are nonparallel for any $v \in V \backslash\{a, b\}$ from the lemma assumption, there exists a small $\varepsilon>0$ such that $\Pi^{t}(a)$ and $\Pi_{G, \mathbf{p}}(v)$ are nonparallel within $-\varepsilon<t<\varepsilon$. Hence, the following mapping $\mathbf{p}^{t}$ on $E$ is well-defined within $-\varepsilon<t<\varepsilon$ :

$$
\mathbf{p}^{t}(e)= \begin{cases}\mathbf{p}(e) & \text { if } e \in E \backslash \delta_{G}(a) \cup\{a b\}, \\ \Pi^{t}(a) \cap \Pi_{G, \mathbf{p}}(v) & \text { if } e=a v \in \delta_{G}(a) \backslash\{a b\} .\end{cases}
$$

Notice that $\mathbf{p}^{0}(e)=\mathbf{p}(e)$ for all $e \in E$ and $\left(G, \mathbf{p}^{0}\right)=(G, \mathbf{p})$ holds. Also, $\left(G, \mathbf{p}^{t}\right)$ is a nonparallel panel-hinge realization for any $0<t<\varepsilon$. Since each $\mathbf{p}^{t}(e), e \in E$ moves continuously with respect to $t$, each minor of $R\left(G, \mathbf{p}^{t}\right)$ can be described as a continuous function of $t$ within $-\varepsilon<t<\varepsilon$. This implies that there exists a small $\varepsilon^{\prime}$ with $0<\varepsilon^{\prime} \leq \varepsilon$ such that $\left(G, \mathbf{p}^{t}\right)$ is a nonparallel panel-hinge realization satisfying $\operatorname{rank} R\left(G, \mathbf{p}^{t}\right) \geq \operatorname{rank} R\left(G, \mathbf{p}^{0}\right)$ for any $0<t<\varepsilon^{\prime}$.

\subsection{Molecular Conjecture}

We now start to show our main result. We first claim the rigidity of graphs consisting of a small number of vertices since it will be used several times (including the base case of the induction).

Lemma 5.3 Let $G=(V, E)$ be the graph consisting of two vertices $\{u, v\}$ and two parallel edges $\{e, f\}$ between $u$ and $v$. Then, $G$ can be realized as an infinitesimally rigid panel-hinge framework $(G, \mathbf{p})$ such that $\Pi_{G, \mathbf{p}}(u)=\Pi_{G, \mathbf{p}}(v)$. In particular, $\operatorname{rank} R(G, \mathbf{p} ;\{e, f\}, v)=D$ holds if $\mathbf{p}(e) \neq \mathbf{p}(f)$. 
Proof A $(d-1)$-extensor of a $(d-2)$-dimensional affine space is uniquely determined up to a scalar multiplication. This implies that the two extensors $C(\mathbf{p}(e))$ and $C(\mathbf{p}(f))$ span the same vector space if and only if $\mathbf{p}(e)=\mathbf{p}(f)$. Therefore, if $\mathbf{p}(e) \neq \mathbf{p}(f)$, the orthogonal complements of the vector spaces spanned by $C(\mathbf{p}(e))$ and $C(\mathbf{p}(f))$, that is, the row spaces of $r(\mathbf{p}(e))$ and $r(\mathbf{p}(f))$ are distinct. We hence have $\operatorname{rank} R(G, \mathbf{p} ;\{e, f\}, v)=D$ by $\operatorname{rank} R(G, \mathbf{p} ; e, v)=\operatorname{rank} r(\mathbf{p}(e))=D-1$. Since we can realize $G$ as a framework $(G, \mathbf{p})$ such that $\Pi_{G, \mathbf{p}}(u)=\Pi_{G, \mathbf{p}}(v)$ and $\mathbf{p}(e) \neq \mathbf{p}(f)$, the statement follows.

If $G$ is a cycle graph, its realization can be easily analyzed directly from the definition of infinitesimal motions. The detailed calculation can be seen in [4, Proposition 3.4] or [34, Proposition 3] for the 3-dimensional case and the technique can apply to the general dimensional case without any modification.

Lemma 5.4 $[4,34]$ Let $G=(V, E)$ be a cycle graph with $3 \leq|V| \leq D$. Then, $G$ can be realized as an infinitesimally rigid nonparallel panel-hinge framework $(G, \mathbf{p})$.

Let us state the main theorem of this paper.

Theorem 5.5 Let $G=(V, E)$ be a minimal $k$-dof-graph with $|V| \geq 2$ for some nonnegative integer $k$. Then, there exists a (nonparallel, if $G$ is simple) panel-hinge realization $(G, \mathbf{p})$ in $\mathbb{R}^{d}$ satisfying $\operatorname{rank} R(G, \mathbf{p})=D(|V|-1)-k$.

Since the proof is quite long, let us first write up a corollary which follows from Theorem 5.5. The following theorem proves the Molecular Conjecture in a strong sense combined with Proposition 2.3.

Theorem 5.6 Let $G=(V, E)$ be a multigraph. Then, $G$ can be realized as a panelhinge framework $(G, \mathbf{p})$ in $\mathbb{R}^{d}$ which satisfies $\operatorname{rank} R(G, \mathbf{p})=D(|V|-1)-\operatorname{def}(\widetilde{G})$.

Proof Let $k=\operatorname{def}(\widetilde{G})$. By Proposition 2.3, we have $\operatorname{rank} R(G, \mathbf{p}) \leq D(|V|-1)-k$ for any realization $(G, \mathbf{p})$ of $G$. When $G$ is not a minimal $k$-dof-graph, we can remove some edges from $G$ keeping the deficiency of $\widetilde{G}$ so that the resulting graph becomes a minimal $k$-dof-graph. Let $G^{\prime}=\left(V, E^{\prime}\right)$ be the obtained minimal $k$-dofgraph. (If $G$ is a minimal $k$-dof-graph, then let $G^{\prime}=G$.) By Theorem 5.5 , there is a panel-hinge realization $\left(G^{\prime}, \mathbf{q}\right)$ satisfying $R\left(G^{\prime}, \mathbf{q}\right)=D(|V|-1)-k$. Moreover, since the infinitesimal rigidity is projectively invariant (see e.g., [4, Sect. 3.6]), we may assume $\Pi_{G^{\prime}, \mathbf{q}}(u) \cap \Pi_{G^{\prime}, \mathbf{q}}(v) \neq \emptyset$ for every $u, v \in V$. Namely, for any $u, v \in V, \Pi_{G^{\prime}, \mathbf{q}}(u) \cap \Pi_{G^{\prime}, \mathbf{q}}(v)$ contains a $(d-2)$-dimensional affine subspace. Define a mapping $\mathbf{p}$ on $E$ such that $\mathbf{p}(u v)=\mathbf{q}(u v)$ for $u v \in E^{\prime}$ and otherwise $\mathbf{p}(u v)$ is a $(d-2)$-dimensional affine subspace contained in $\Pi_{G^{\prime}, \mathbf{q}}(u) \cap \Pi_{G^{\prime}, \mathbf{q}}(v)$. It is obvious that $(G, \mathbf{p})$ is a panel-hinge realization of $G$ and moreover $\operatorname{rank} R(G, \mathbf{p}) \geq$ $\operatorname{rank} R\left(G^{\prime}, \mathbf{q}\right)=D(|V|-1)-k$. We thus obtain a panel-hinge realization satisfying $\operatorname{rank} R(G, \mathbf{p})=D(|V|-1)-k$. 
Let $(G, \mathbf{p})$ be a nonparallel panel-hinge framework in $\mathbb{R}^{3}$. We can associate a body-hinge framework $\left(G, \mathbf{p}^{*}\right)$ with $(G, \mathbf{p})$, where $\mathbf{p}^{*}(e)$ is dual to $\mathbf{p}(e)$ (when extending to the real projective 3 -space). Then, $\left(G, \mathbf{p}^{*}\right)$ has a "hinge-concurrent" property such that, for any $v \in V$ and any $e \in \delta_{G}(v), \mathbf{p}^{*}(e)$ passes through the point $\mathbf{c}(v)$ (defined in Sect. 5.1). Body-hinge frameworks satisfying the hinge-concurrent property are called molecular frameworks $[13,34]$ since they are used to study the flexibility of molecules. Since the infinitesimal rigidity is invariant under a projective transformation (see [4, Sect. 3.6] or [30] for more detailed descriptions on this topic), it follows from Theorem 5.6 that a simple graph $G=(V, E)$ can be realized as a molecular framework $(G, \mathbf{p})$ which satisfies $\operatorname{rank} R(G, \mathbf{p})=D(|V|-1)-\operatorname{def}(\widetilde{G})$.

Let us consider bar-joint rigidity in 3-dimensional space. For a graph $G$ of the minimum degree at least two, Whiteley showed in [35] that $G$ can be realized as a rigid molecular framework if and only if $G^{2}$ can be realized as an infinitesimally rigid bar-joint framework. Jackson and Jordán proved in [10, 13] that the Molecular Conjecture (Conjecture 1.2) is equivalent to the following statement:

Corollary 5.7 Let $G=(V, E)$ be a graph with minimum degree at least two. Then $r\left(G^{2}\right)=3|V|-6-\operatorname{def}(\widetilde{G})$, where $r$ denotes the rank function of the 3-dimensional generic rigidity matroid.

Further combinatorial results on 3-dimensional bar-joint frameworks of square graphs can be found in $[10,13]$.

\section{Proof of Theorem 5.5}

The proof is done by induction on $|V|$. Let us consider the base case $|V|=2$. Let $V=\{u, v\}$. By Lemma 3.2, any minimal $k$-dof-graph is not 3-edge-connected and hence we have three possible cases: (i) $E$ is empty, (ii) $E$ consists of a single edge $e$ connecting $u$ and $v$, and (iii) $E$ consists of two parallel edges $\{e, f\}$ between $u$ and $v$. The cases (i) and (ii) are trivial since any realization satisfies the statement. The case (iii) has been treated in Lemma 5.3.

Let us consider $G$ with $|V| \geq 3$. We shall split the proof into four cases:

- $G$ is not 2-edge-connected (Sect. 6.1).

- $G$ contains a proper rigid subgraph (Sect. 6.2).

- $G$ is 2-edge-connected, contains no proper rigid subgraph, and $k>0$ (Sect. 6.3).

- $G$ is 2-edge-connected, contains no proper rigid subgraph, and $k=0$ (Sect. 6.4).

In each case, we will assume the following induction hypothesis on $|V|$ :

For any minimal $k_{H}$-dof-graph $H=\left(V_{H}, E_{H}\right)$ for some nonnegative integer $k_{H}$ with $\left|V_{H}\right|<|V|$, there is a (nonparallel, if $G_{H}$ is simple) panel-hinge realization $\left(G_{H}, \mathbf{p}_{H}\right)$ in $\mathbb{R}^{d}$ satisfying $\operatorname{rank} R\left(G_{H}, \mathbf{p}_{H}\right)=D\left(\left|V_{H}\right|-1\right)-k_{H}$. 


\section{$6.1 G$ is Not 2-edge-connected}

This case is handled rather easily.

Lemma 6.1 Let $G=(V, E)$ be a minimal $k$-dof-graph that is not 2-edge-connected. Suppose that (6.1) holds. Then, there is a (nonparallel, if $G$ is simple) panel-hinge realization $(G, \mathbf{p})$ in $\mathbb{R}^{d}$ satisfying $\operatorname{rank} R(G, \mathbf{p})=D(|V|-1)-k$.

Proof Let us consider the case where $G$ is connected. Since $G$ has a cut edge $u v$, $G$ can be partitioned into two subgraphs $G_{1}=\left(V_{1}, E_{1}\right)$ and $G_{2}=\left(V_{2}, E_{2}\right)$ such that $u \in V_{1}, v \in V_{2}, V_{1} \cap V_{2}=\emptyset, V_{1} \cup V_{2}=V$ and $\delta_{G}\left(\left\{V_{1}, V_{2}\right\}\right)=\{u v\}$. Let $k_{1}$ and $k_{2}$ be the deficiencies of $\widetilde{G_{1}}$ and $\widetilde{G_{2}}$, respectively. Then, $k=k_{1}+k_{2}+1$ holds by Lemma 3.6 and also $G_{i}$ is a minimal $k_{i}$-dof-graph for each $i=1,2$ by Lemma 3.3. By (6.1), we have a (nonparallel, if $G_{i}$ is simple) panel-hinge realization $\left(G_{i}, \mathbf{p}_{i}\right)$ satisfying rank $R\left(G_{i}, \mathbf{p}_{i}\right)=D\left(\left|V_{i}\right|-1\right)-k_{i}$ for each $i=1,2$. Since the choices of $\mathbf{p}_{1}$ and $\mathbf{p}_{2}$ are independent of each other and also since the rank of the rigidity matrix is invariant under an isometric transformation of the whole framework, we can take $\mathbf{p}_{1}$ and $\mathbf{p}_{2}$ such that $\Pi_{G_{1}, \mathbf{p}_{1}}\left(v_{1}\right)$ and $\Pi_{G_{2}, \mathbf{p}_{2}}\left(v_{2}\right)$ are nonparallel for any pair of $v_{1} \in V_{1}$ and $v_{2} \in V_{2}$. In particular, $\Pi_{G_{1}, \mathbf{p}_{1}}(u) \cap \Pi_{G_{2}, \mathbf{p}_{2}}(v)$ is a $(d-2)$-dimensional affine subspace in $\mathbb{R}^{d}$. Define a mapping $\mathbf{p}$ as

$$
\mathbf{p}(e)= \begin{cases}\mathbf{p}_{1}(e) & \text { if } e \in E_{1}, \\ \mathbf{p}_{2}(e) & \text { if } e \in E_{2}, \\ \Pi_{G_{1}, \mathbf{p}_{1}}(u) \cap \Pi_{G_{2}, \mathbf{p}_{2}}(v) & \text { if } e=u v .\end{cases}
$$

Then, $(G, \mathbf{p})$ is a (nonparallel, if $G$ is simple) panel-hinge realization of $G$. By $\delta_{G}\left(\left\{V_{1}, V_{2}\right\}\right)=\{u v\}$, the rigidity matrix $R(G, \mathbf{p})$ can be described as

$$
\begin{aligned}
& R(G, \mathbf{p})={ }_{u v}^{E_{1}}\left(\frac{R\left(G_{1}, \mathbf{p}_{1}\right) \mid}{V_{E_{2}}}\left(\begin{array}{c}
V_{2} \\
R(G, \mathbf{p} ; u v)
\end{array}\right)\right. \\
& =\begin{array}{c}
E_{1} \\
u v \\
E_{2}
\end{array}\left(\begin{array}{c|c|c|c}
R\left(G_{1}, \mathbf{p}_{1} ; E_{1}, V_{1} \backslash\{u\}\right) & R\left(G_{1}, \mathbf{p}_{1} ; E_{1}, u\right) & \mathbf{0} & \mathbf{0} \\
\hline \mathbf{0} & r(\mathbf{p}(u v)) & -r(\mathbf{p}(u v)) & \mathbf{0} \\
\hline \mathbf{0} & \mathbf{0} & R\left(G_{2}, \mathbf{p}_{2}\right)
\end{array}\right) .
\end{aligned}
$$

Notice that $\operatorname{rank} R\left(G_{1}, \mathbf{p}_{1} ; E_{1}, V_{1} \backslash\{u\}\right)=\operatorname{rank} R\left(G_{1}, \mathbf{p}\right)=D\left(\left|V_{1}\right|-1\right)-k_{1}$ holds by Lemma 5.1. Note also $\operatorname{rank} r(\mathbf{p}(u v))=D-1$ from the definition. Hence, by $k=k_{1}+k_{2}+1$ and $|V|=\left|V_{1}\right|+\left|V_{2}\right|$, we obtain $\operatorname{rank} R(G, \mathbf{p}) \geq \operatorname{rank} R\left(G_{1}, \mathbf{p}_{1} ; E_{1}\right.$, $\left.V_{1} \backslash\{u\}\right)+\operatorname{rank} r(\mathbf{p}(u v))+\operatorname{rank} R\left(G_{2}, \mathbf{p}_{2}\right)=D\left(\left|V_{1}\right|-1\right)-k_{1}+(D-1)+$ $D\left(\left|V_{2}\right|-1\right)-k_{2}=D(|V|-1)-\left(k_{1}+k_{2}+1\right)=D(|V|-1)-k$.

The proof for disconnected graphs $G$ can be proved in the same manner. 


\subsection{G Contains a Proper Rigid Subgraph}

Let us first describe a proof sketch. Let $G^{\prime}=\left(V^{\prime}, E^{\prime}\right)$ be a proper rigid subgraph in a minimal $k$-dof-graph $G=(V, E)$. Note that $G^{\prime}$ is a minimal 0-dof-graph by Lemma 3.3 with $1<\left|V^{\prime}\right|<|V|$. Let $G / E^{\prime}=\left(\left(V \backslash V^{\prime}\right) \cup\left\{v^{*}\right\}, E \backslash E^{\prime}\right)$ be the graph obtained from $G$ by contracting the edges of $E^{\prime}$, where $v^{*}$ is the new vertex obtained by the contraction. Then, by Lemma $3.5, G / E^{\prime}$ is a minimal $k$-dofgraph with $\left|\left(V \backslash V^{\prime}\right) \cup\left\{v^{*}\right\}\right|<|V|$. Therefore, by induction, there exist panel-hinge realizations $\left(G^{\prime}, \mathbf{p}_{1}\right)$ and $\left(G / E^{\prime}, \mathbf{p}_{2}\right)$ satisfying $\operatorname{rank} R\left(G^{\prime}, \mathbf{p}_{1}\right)=D\left(\left|V^{\prime}\right|-1\right)$ and $\operatorname{rank} R\left(G / E^{\prime}, \mathbf{p}_{2}\right)=D\left(\left|V \backslash V^{\prime} \cup\left\{v^{*}\right\}\right|-1\right)-k$. Based on these realizations, we shall construct a realization of $G$. Intuitively, we shall replace the body associated with $v^{*}$ in $\left(G / E^{\prime}, \mathbf{p}_{2}\right)$ by $\left(G^{\prime}, \mathbf{p}_{1}\right)$, by regarding $\left(G^{\prime}, \mathbf{p}_{1}\right)$ as a rigid body in $\mathbb{R}^{d}$ and show that the rank of the resulting framework becomes $\operatorname{rank} R\left(G^{\prime}, \mathbf{p}_{1}\right)+\operatorname{rank} R\left(G / E^{\prime}, \mathbf{p}_{2}\right)$, which is equal to $D(|V|-1)-k$. In order to obtain a nonparallel realization if $G$ is simple, we need three subcases. Lemma 6.2 deals with the case where $G$ is not a simple graph. Lemma 6.3 deals with the case where $G$ is simple and there exists a proper rigid subgraph $G^{\prime}=\left(V^{\prime}, E^{\prime}\right)$ such that $G / E^{\prime}$ is also simple. Lemma 6.5 deals with the rest of the cases.

Lemma 6.2 Let $G=(V, E)$ be a minimal $k$-dof-graph with $|V| \geq 3$. Suppose that $G$ is not simple and also that (6.1) holds. Then, there is a panel-hinge realization $(G, \mathbf{p})$ in $\mathbb{R}^{d}$ satisfying $\operatorname{rank} R(G, \mathbf{p})=D(|V|-1)-k$.

Proof Let $e$ and $f$ be multiple edges connecting $a$ and $b$ for some $a, b \in V$. Then, notice that the graph $G[\{e, f\}]$ edge-induced by $\{e, f\}$ is a proper rigid subgraph in $G$ since $\widetilde{e} \cup \widetilde{f}$ contains $D$ edge-disjoint spanning trees on $\{a, b\}$. Hence we can assume $G^{\prime}=G[\{e, f\}]=\left(V^{\prime}=\{a, b\}, E^{\prime}=\{e, f\}\right)$. By Lemma 5.3, there exists a panel-hinge realization $\left(G^{\prime}, \mathbf{p}_{1}\right)$ of $G^{\prime}$ such that $\operatorname{rank} R\left(G^{\prime}, \mathbf{p}_{1}\right)=D$ and $\Pi_{G^{\prime}, \mathbf{p}_{1}}(a)=$ $\Pi_{G^{\prime}, \mathbf{p}_{1}}(b)$. Also, by (6.1), there exists a panel-hinge realization $\left(G / E^{\prime}, \mathbf{p}_{2}\right)$ satisfying $\operatorname{rank} R\left(G / E^{\prime}, \mathbf{p}_{2}\right)=D(|V|-2)-k$. Since the choices of $\mathbf{p}_{1}$ and $\mathbf{p}_{2}$ are independent of each other, we can take $\mathbf{p}_{1}$ and $\mathbf{p}_{2}$ in such a way that $\Pi_{G / E^{\prime}, \mathbf{p}_{2}}\left(v^{*}\right)=\Pi_{G^{\prime}, \mathbf{p}_{1}}(a)=$ $\Pi_{G^{\prime}, \mathbf{p}_{1}}(b)$. Define a mapping on $E$ as

$$
\mathbf{p}(e)= \begin{cases}\mathbf{p}_{1}(e) & \text { if } e \in E^{\prime}(=\{e, f\}) \\ \mathbf{p}_{2}(e) & \text { if } e \in E \backslash E^{\prime}\end{cases}
$$

Intuitively, $(G, \mathbf{p})$ is a panel-hinge realization of $G$ obtained from $\left(G / E^{\prime}, \mathbf{p}_{2}\right)$ by identifying the panels of $a$ and $b$ with that of $v^{*}$, and in fact it can be easily verified from the definition that $(G, \mathbf{p})$ is a panel-hinge realization satisfying $\Pi_{G, \mathbf{p}}(u)=$ $\Pi_{G / E^{\prime}, \mathbf{p}_{2}}(u)$ for each $u \in V \backslash\{a, b\}$ and $\Pi_{G, \mathbf{p}}(u)=\Pi_{G / E^{\prime}, \mathbf{p}_{2}}\left(v^{*}\right)$ for each $u \in\{a, b\}$.

Let us take a look at the rigidity matrix $R(G, \mathbf{p})$ :

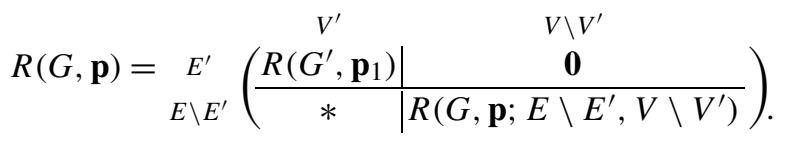


Since $\mathbf{p}(e)=\mathbf{p}_{2}(e)$ for every $e \in E \backslash E^{\prime}$ by (6.2), we can take ${ }^{5}$ the entries of $R\left(G, \mathbf{p} ; E \backslash E^{\prime}, V \backslash V^{\prime}\right)$ to be

$$
R\left(G, \mathbf{p} ; E \backslash E^{\prime}, V \backslash V^{\prime}\right)=R\left(G / E^{\prime}, \mathbf{p}_{2} ; E \backslash E^{\prime}, V \backslash V^{\prime}\right) .
$$

We also remark that $R\left(G / E^{\prime}, \mathbf{p}_{2} ; E \backslash E^{\prime}, V \backslash V^{\prime}\right)$ is the matrix obtained from $R\left(G / E^{\prime}, \mathbf{p}_{2}\right)$ by deleting the $D$ consecutive columns associated with $v^{*}$. Hence, by (6.4) and Lemma 5.1, we obtain

$$
\begin{aligned}
\operatorname{rank} R\left(G, \mathbf{p} ; E \backslash E^{\prime}, V \backslash V^{\prime}\right) & =\operatorname{rank} R\left(G / E^{\prime}, \mathbf{p}_{2} ; E \backslash E^{\prime}, V \backslash V^{\prime}\right) \\
& =\operatorname{rank} R\left(G / E^{\prime}, \mathbf{p}_{2}\right) .
\end{aligned}
$$

As a result, by (6.3) and (6.5), we obtain

$$
\begin{aligned}
\operatorname{rank} R(G, \mathbf{p}) & \geq \operatorname{rank} R\left(G^{\prime}, \mathbf{p}_{1}\right)+\operatorname{rank} R\left(G, \mathbf{p} ; E \backslash E^{\prime}, V \backslash V^{\prime}\right) \\
& =\operatorname{rank} R\left(G^{\prime}, \mathbf{p}_{1}\right)+\operatorname{rank} R\left(G / E^{\prime}, \mathbf{p}_{2}\right) \\
& =D+D(|V|-2)-k=D(|V|-1)-k .
\end{aligned}
$$

Lemma 6.3 Let $G=(V, E)$ be a minimal $k$-dof-graph with $|V| \geq 3$. Suppose that $G$ is simple and contains a proper rigid subgraph $G^{\prime}=\left(V^{\prime}, E^{\prime}\right)$ such that $G / E^{\prime}$ is simple. Also suppose that (6.1) holds. Then, there exists a nonparallel panel-hinge realization $(G, \mathbf{p})$ satisfying $\operatorname{rank} R(G, \mathbf{p})=D(|V|-1)-k$.

Proof The story of the proof is basically the same as that of Lemma 6.2 although we need a slightly different mapping p. By Lemma 3.5 and (6.1), there exist nonparallel panel-hinge realizations $\left(G^{\prime}, \mathbf{p}_{1}\right)$ and $\left(G / E^{\prime}, \mathbf{p}_{2}\right)$ satisfying $\operatorname{rank} R\left(G^{\prime}, \mathbf{p}_{1}\right)=$ $D\left(\left|V^{\prime}\right|-1\right)$ and $\operatorname{rank} R\left(G / E^{\prime}, \mathbf{p}_{2}\right)=D\left(\left|V \backslash V^{\prime} \cup\{v\}\right|-1\right)-k$. From the definition of generic nonparallel panel-hinge realizations of simple graphs discussed in Sect. 5.1, $\mathbf{p}_{1}$ and $\mathbf{p}_{2}$ can be taken in such a way that the set of all coefficients appearing in the equations expressing the hyperplanes $\Pi_{G^{\prime}, \mathbf{p}_{1}}\left(v_{1}\right)$ for all $v_{1} \in V^{\prime}$ and $\Pi_{G / E^{\prime}, \mathbf{p}_{2}}\left(v_{2}\right)$ for all $v_{2} \in V \backslash V^{\prime}$ is algebraically independent over $\mathbb{Q}$. We define a mapping $\mathbf{p}$ on $E$ as follows:

$$
\mathbf{p}(e)= \begin{cases}\mathbf{p}_{1}(e) & \text { if } e \in E^{\prime} \\ \mathbf{p}_{2}(e) & \text { if } e \in E \backslash\left(E^{\prime} \cup \delta_{G}\left(V^{\prime}\right)\right) \\ \Pi_{G / E^{\prime}, \mathbf{p}_{2}}(u) \cap \Pi_{G^{\prime}, \mathbf{p}_{1}}(v) & \text { if } e=u v \in \delta_{G}\left(V^{\prime}\right) \text { with } u \in V \backslash V^{\prime}, v \in V^{\prime} .\end{cases}
$$

Then, $(G, \mathbf{p})$ is a nonparallel panel-hinge realization of $G$ since all $\mathbf{p}(e)$ for $e \in$ $\delta_{G}\left(v_{1}\right)$ are contained in $\Pi_{G^{\prime}, \mathbf{p}_{1}}\left(v_{1}\right)$ for each $v_{1} \in V^{\prime}$ and all $\mathbf{p}(e)$ for $e \in \delta_{G}\left(v_{2}\right)$ are contained in $\Pi_{G / E^{\prime}, \mathbf{p}_{2}}\left(v_{2}\right)$ for each $v_{2} \in V \backslash V^{\prime}$. Consider the rigidity matrix $R(G, \mathbf{p})$, which can be described in the same way as (6.3). We shall derive

\footnotetext{
${ }^{5}$ Recall the discussion in Sect. 2.4; the entries of the rigidity matrix are not uniquely defined and depend on the choice of a basis of the orthogonal complement of the vector space spanned by each $(d-1)$-extensor although the null space of the rigidity matrix is determined uniquely.
} 
$\operatorname{rank} R\left(G, \mathbf{p} ; E \backslash E^{\prime}, V \backslash V^{\prime}\right)=\operatorname{rank} R\left(G / E^{\prime}, \mathbf{p}_{2}\right)$ as was done in the proof of the previous lemma. This will prove $\operatorname{rank} R(G, \mathbf{p})=D(|V|-1)-k$ as before. To obtain this relation, we shall compare $\mathbf{p}_{2}$ with the restriction $\mathbf{p}_{E \backslash E^{\prime}}$ of $\mathbf{p}$ on $E \backslash E^{\prime}$, that is,

$\mathbf{p}_{E \backslash E^{\prime}}(e)= \begin{cases}\mathbf{p}_{2}(e) & \text { if } e \in E \backslash\left(E^{\prime} \cup \delta_{G}\left(V^{\prime}\right)\right) \\ \Pi_{G / E^{\prime}, \mathbf{p}_{2}}(u) \cap \Pi_{G^{\prime}, \mathbf{p}_{1}}(v) & \text { if } e=u v \in \delta_{G}\left(V^{\prime}\right) \text { with } u \in V \backslash V^{\prime}, v \in V^{\prime} .\end{cases}$

The body-hinge framework $\left(G / E^{\prime}, \mathbf{p}_{E \backslash E^{\prime}}\right)$ is not a panel-hinge realization in general; all hinges $\mathbf{p}_{E \backslash E^{\prime}}(e)$ of $e \in \delta_{G / E^{\prime}}(u)$ are contained in the panel $\Pi_{G / E^{\prime}, \mathbf{p}_{2}}(u)$ for each $u \in V \backslash V^{\prime}$ while the hinges $\mathbf{p}_{E \backslash E^{\prime}}(e)$ of $e \in \delta_{G / E^{\prime}}\left(v^{*}\right)$ may not be on a panel. Intuitively, $\left(G / E^{\prime}, \mathbf{p}_{E \backslash E^{\prime}}\right)$ is a body-hinge realization of $G / E^{\prime}$ such that every $v \in V \backslash V^{\prime}$ is realized as a panel $\Pi_{G / E^{\prime}, \mathbf{p}_{2}}(v)$ while $v^{*}$ may be realized as a $d$-dimensional body.

Claim 6.4 $\operatorname{rank} R\left(G / E^{\prime}, \mathbf{p}_{E \backslash E^{\prime}}\right) \geq \operatorname{rank} R\left(G / E^{\prime}, \mathbf{p}_{2}\right)$ holds.

Proof As mentioned in [15] and also in Sect. 5.1, each entry of the rigidity matrix of a nonparallel realization can be written in terms of the coefficients appearing in the equations representing the panels associated with $v \in V$. Although $\left(G / E^{\prime}, \mathbf{p}_{E \backslash E^{\prime}}\right)$ is not a panel-hinge realization, due to the definition (6.7), each entry of $R\left(G / E^{\prime}, \mathbf{p}_{E \backslash E^{\prime}}\right)$ is also described in terms of the coefficients appearing in the equations expressing $\Pi_{G^{\prime}, \mathbf{p}_{1}}\left(v_{1}\right)$ for $v_{1} \in V^{\prime}$ and $\Pi_{G / E^{\prime}, \mathbf{p}_{2}}\left(v_{2}\right)$ for $v_{2} \in V \backslash V^{\prime}$. Notice that the collection of all the nonparallel panel-hinge realizations of $G / E^{\prime}$ is a subset of the collection of all possible realizations of $G / E^{\prime}$ determined by (6.7) for some choices of $\mathbf{p}_{1}$ and $\mathbf{p}_{2}$, because for any $\left(G / E^{\prime}, \mathbf{p}_{2}\right)$ we can obtain $\left(G / E^{\prime}, \mathbf{p}_{E \backslash E^{\prime}}\right)=\left(G / E^{\prime}, \mathbf{p}_{2}\right)$ by setting $\Pi_{G^{\prime}, \mathbf{p}_{1}}(u)$ to be $\Pi_{G / E^{\prime}, \mathbf{p}_{2}}\left(v^{*}\right)$ for all $u \in V^{\prime}$. This implies rank $R\left(G / E^{\prime}, \mathbf{p}_{E \backslash E^{\prime}}\right) \geq \operatorname{rank} R\left(G / E^{\prime}, \mathbf{p}_{2}\right)$ since $\mathbf{p}_{1}$ and $\mathbf{p}_{2}$ have been taken in such a way that the set of all coefficients appearing in the equations representing the panels is algebraically independent over $\mathbb{Q}$ (and hence $R\left(G / E^{\prime}, \mathbf{p}_{E \backslash E^{\prime}}\right.$ ) takes the maximum rank over all realizations of $G / E^{\prime}$ determined by (6.7)).

Let us take a look at the rigidity matrix $R(G, \mathbf{p})$ written in (6.3). Since $\mathbf{p}_{E \backslash E^{\prime}}$ is the restriction of $\mathbf{p}$ on $E \backslash E^{\prime}$, we have

$$
R\left(G, \mathbf{p} ; E \backslash E^{\prime}, V \backslash V^{\prime}\right)=R\left(G / E^{\prime}, \mathbf{p}_{E \backslash E^{\prime}} ; E \backslash E^{\prime}, V \backslash V^{\prime}\right) .
$$

We remark that $R\left(G / E^{\prime}, \mathbf{p}_{E \backslash E^{\prime}} ; E \backslash E^{\prime}, V \backslash V^{\prime}\right)$ is the matrix obtained from $R\left(G / E^{\prime}, \mathbf{p}_{E \backslash E^{\prime}}\right)$ by deleting the $D$ consecutive columns associated with $v^{*}$. Therefore, by (6.8), Lemma 5.1, and Claim 6.4, we obtain

$$
\begin{aligned}
\operatorname{rank} R\left(G, \mathbf{p} ; E \backslash E^{\prime}, V \backslash V^{\prime}\right) & =\operatorname{rank} R\left(G / E^{\prime}, \mathbf{p}_{E \backslash E^{\prime}} ; E \backslash E^{\prime}, V \backslash V^{\prime}\right) \\
& =\operatorname{rank} R\left(G / E^{\prime}, \mathbf{p}_{E \backslash E^{\prime}}\right) \\
& \geq \operatorname{rank} R\left(G / E^{\prime}, \mathbf{p}_{2}\right) .
\end{aligned}
$$

By (6.3) and (6.9), we eventually obtain $\operatorname{rank} R(G, \mathbf{p}) \geq \operatorname{rank} R\left(G^{\prime}, \mathbf{p}_{1}\right)+$ $\operatorname{rank} R\left(G, \mathbf{p} ; E \backslash E^{\prime}, V \backslash V^{\prime}\right) \geq \operatorname{rank} R\left(G^{\prime}, \mathbf{p}_{1}\right)+\operatorname{rank} R\left(G / E^{\prime}, \mathbf{p}_{2}\right)=D\left(\left|V^{\prime}\right|-1\right)+$ $D\left(\left|V \backslash V^{\prime} \cup\left\{v^{*}\right\}\right|-1\right)-k=D(|V|-1)-k$. 
Lemma 6.5 Let $G$ be a minimal $k$-dof-graph with $|V| \geq 3$ that contains a proper rigid subgraph. Suppose that $G$ is simple but contains no proper rigid subgraph $G^{\prime}=$ $\left(V^{\prime}, E^{\prime}\right)$ such that $G / E^{\prime}$ is simple. Also suppose that (6.1) holds. Then, there exists a nonparallel panel-hinge realization $(G, \mathbf{p})$ satisfying $\operatorname{rank} R(G, \mathbf{p})=D(|V|-1)-k$.

Proof We shall consider a different approach from those of the previous lemmas, based on the following observation.

Claim 6.6 $G$ is a 0 -dof-graph and there exists a vertex $v$ of degree two such that $G_{v}$ is a minimal 0-dof-graph, where $G_{v}$ is the graph obtained from $G$ by the removal of $v$.

Proof Let us take a vertex-inclusionwise maximal proper rigid subgraph $G^{\prime}=$ $\left(V^{\prime}, E^{\prime}\right)$ of $G$. Note that $G^{\prime}$ is a minimal 0-dof-graph by Lemma 3.3. Since $G / E^{\prime}$ is not simple, there exist a vertex $v \in V \backslash V^{\prime}$ and two edges, denoted by $e$ and $f$, which are incident to $v$ and some other vertices of $V^{\prime}$. Let $G^{\prime \prime}=\left(V^{\prime} \cup\{v\}, E^{\prime} \cup\{e, f\}\right)$. Then, $G^{\prime}$ is the graph obtained from $G^{\prime \prime}$ by the removal of $v$. By Lemma 4.4, we obtain $\operatorname{def}\left(\widetilde{G^{\prime \prime}}\right) \leq \operatorname{def}\left(\widetilde{G}^{\prime}\right)=0$. Hence $G^{\prime \prime}$ is also a 0 -dof-graph and equivalently a rigid subgraph of $G$. Since $G^{\prime}$ is taken as a vertex-inclusionwise maximal proper rigid subgraph of $G, G^{\prime \prime}$ cannot be a proper rigid subgraph and consequently $V=V^{\prime} \cup\{v\}$. This implies that $G$ is a 0 -dof-graph. Also, the minimality of $G$ implies $G=G^{\prime \prime}$. Since $v$ is a vertex of degree two in $G^{\prime \prime}=G$, the removal of $v$ from $G$ results in the minimal 0-dof-graph $G^{\prime}$.

Let $v$ be a vertex of degree two whose removal results in a minimal 0-dof-graph $G_{v}$ as shown in Claim 6.6. Let $N_{G}(v)=\{a, b\}$. Note that $G_{v}$ is simple since $G$ is simple. Hence, by (6.1), there exists a nonparallel panel-hinge realization $\left(G_{v}, \mathbf{q}\right)$ satisfying $\operatorname{rank} R\left(G_{v}, \mathbf{q}\right)=D(|V \backslash\{v\}|-1)$. We define a mapping $\mathbf{p}$ on $E$ as follows:

$$
\mathbf{p}(e)= \begin{cases}\mathbf{q}(e) & \text { if } e \in E \backslash\{v a, v b\} \\ \Pi^{\circ} \cap \Pi_{G_{v}, \mathbf{q}}(a) & \text { if } e=v a \\ \Pi^{\circ} \cap \Pi_{G_{v}, \mathbf{q}}(b) & \text { if } e=v b\end{cases}
$$

where $\Pi^{\circ}$ is a $(d-1)$-dimensional affine subspace that is not parallel to $\Pi_{G_{v}, \mathbf{q}}(u)$ for $u \in V \backslash\{v\}$ and does not contain $\Pi_{G_{v}, \mathbf{q}}(a) \cap \Pi_{G_{v}, \mathbf{q}}(b)$ (so that $\Pi^{\circ} \cap \Pi_{G_{v}, \mathbf{q}}(a) \neq$ $\left.\Pi^{\circ} \cap \Pi_{G_{v}, \mathbf{q}}(b)\right)$. Clearly, $(G, \mathbf{p})$ is a nonparallel panel-hinge realization. Let us take a look at the rigidity matrix $R(G, \mathbf{p})$ :

$$
R(G, \mathbf{p})=\begin{gathered}
v a \\
v b \\
E \backslash\{v a, v b\}
\end{gathered}\left(\begin{array}{c|c}
v & V \backslash\{v\} \\
\hline R(G, \mathbf{p} ; v a, v) & * \\
\hline \mathbf{0} & R(G, \mathbf{p} ; E \backslash\{v a, v b\}, V \backslash\{v\})
\end{array}\right) .
$$

Since $G_{v}=(V \backslash\{v\}, E \backslash\{v a, v b\})$ and also $\mathbf{p}(e)=\mathbf{q}(e)$ holds for any $e \in E \backslash$ $\{v a, v b\}$, we can take the entries of the rigidity matrix in such a way that $R(G, \mathbf{p} ; E \backslash$ $\{v a, v b\}, V \backslash\{v\})=R\left(G_{v}, \mathbf{q}\right)$. Also, notice that the top-left $2(D-1) \times D$-submatrix, 
i.e. $R(G, \mathbf{p} ;\{v a, v b\}, v)$, has rank equal to $D$ by Lemma 5.3. We thus obtain $\operatorname{rank} R(G, \mathbf{p}) \geq \operatorname{rank} R(G, \mathbf{p} ;\{v a, v b\}, v)+\operatorname{rank} R(G, \mathbf{p} ; E \backslash\{v a, v b\}, V \backslash\{v\})=$ $D+\operatorname{rank} R\left(G_{v}, \mathbf{q}\right)=D+D(|V \backslash\{v\}|-1)=D(|V|-1)$.

This completes the case where $G$ contains a proper rigid subgraph.

\section{$6.3 G$ is 2-edge-connected, Contains No Proper Rigid Subgraph, and $k>0$}

Let us consider the remaining case for proving Theorem 5.5; $G$ is 2-edge-connected and has no proper rigid subgraph. We shall further split this case into two subcases depending on whether $k>0$ or $k=0$. This subsection deals with the case $k>0$. We need the following two easy observations, which contribute to the claim that $G_{v}^{a b}$ is always simple.

Lemma 6.7 Let $G=(V, E)$ be a 2-edge-connected minimal $k$-dof-graph with $|V| \geq$ 3 for some nonnegative integer $k$. Suppose also that $G$ contains no proper rigid subgraph. Then, the following holds:

(i) If $|V|=3$, then $k=0$ and there is a nomparallel panel-hinge realization $(G, \mathbf{p})$ satisfying $\operatorname{rank} R(G, \mathbf{p})=D(|V|-1)$.

(ii) If $|V| \geq 4$, then $G_{v}^{a b}$ is a simple graph for any vertex $v$ of degree two, where $N_{G}(v)=\{a, b\}$.

Proof We remark that $G$ is simple since multiple edges induce a proper rigid subgraph. When $|V|=3, G$ is a triangle because $G$ is simple and 2-edge-connected. Hence, by Lemma 5.4, there is a nonparallel panel-hinge framework $(G, \mathbf{p})$ satisfying $\operatorname{rank} R(G, \mathbf{p})=D(|V|-1)$.

Let us consider (ii). If $G_{v}^{a b}$ is not simple, then $G_{v}^{a b}$ contains two parallel edges between $a$ and $b$ because the original graph $G$ is simple. This implies $a b \in E$. Therefore $G$ contains a triangle $G[\{v a, v b, a b\}]$ as its subgraph. Since a triangle is a 0 -dof-graph, $G$ contains a proper rigid subgraph, contradicting the lemma assumption.

Lemma 6.8 Let $G=(V, E)$ be a 2-edge-connected minimal $k$-dof-graph with $|V| \geq$ 3 for some integer $k$ with $k>0$. Suppose that there exists no proper rigid subgraph in $G$ and that (6.1) holds. Then, there is a nonparallel panel-hinge realization $(G, \mathbf{p})$ in $\mathbb{R}^{d}$ satisfying $\operatorname{rank} R(G, \mathbf{p})=D(|V|-1)-k$.

Proof By Lemma 4.6, $G$ has a vertex $v$ of degree two with $N_{G}(v)=\{a, b\}$. Since $k>0$ and there is no proper rigid subgraph in $G$, Lemma 4.8 implies that $G_{v}^{a b}$ is a minimal $(k-1)$-dof-graph. Also, by Lemma 6.7, we can assume that $G_{v}^{a b}$ is simple. Hence, by (6.1), there exists a generic nonparallel panel-hinge realization $\left(G_{v}^{a b}, \mathbf{q}\right)$ 


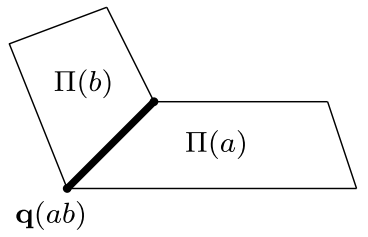

(a)

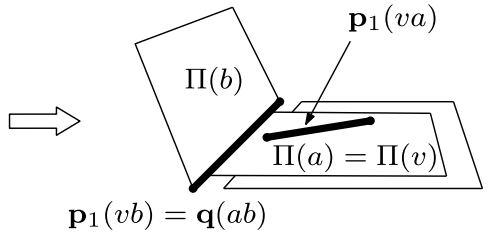

(b)

Fig. 4 The realizations given in the proof of Lemma 6.8 around $v$, where the panels associated with the vertices other than $v, a, b$ are omitted. (a) $\left(G_{v}^{a b}, \mathbf{q}\right)$. (b) $\left(G, \mathbf{p}_{1}\right)$

in $\mathbb{R}^{d}$, which satisfies

$$
\operatorname{rank} R\left(G_{v}^{a b}, \mathbf{q}\right)=D(|V \backslash\{v\}|-1)-(k-1) .
$$

Let $E_{v}=E \backslash\{v a, v b\}$. Define a mapping $\mathbf{p}_{1}$ on $E$ as follows:

$$
\mathbf{p}_{1}(e)= \begin{cases}\mathbf{q}(e) & \text { if } e \in E_{v}(=E \backslash\{v a, v b\}) \\ L & \text { if } e=v a \\ \mathbf{q}(a b) & \text { if } e=v b\end{cases}
$$

where $L$ is a $(d-2)$-dimensional affine subspace contained in $\Pi_{G_{v}^{a b}, \mathbf{q}}(a)$. In Fig. 4, we illustrate this realization. We need to prove the following fact.

Claim $6.9\left(G, \mathbf{p}_{1}\right)$ is a panel-hinge realization of $G$ for any choice of $(d-2)$ dimensional affine subspace $L$ contained in $\Pi_{G_{v}^{a b}, \mathbf{q}}(a)$.

Proof From the definition of $\mathbf{p}_{1}$ and from the fact that $\left(G_{v}^{a b}, \mathbf{q}\right)$ is a panel-hinge realization, every hinge $\mathbf{p}_{1}(e)$ for $e=u w \in E \backslash\{v a, v b\}$ is appropriately contained in the panels $\Pi_{G_{v}^{a b}, \mathbf{q}}(u)$ and $\Pi_{G_{v}^{a b}, \mathbf{q}}(w)$. This implies that, for every $u \in V \backslash\{v, a, b\}$, all the hinges $\mathbf{p}_{1}(e)$ for $e \in \delta_{G}(u)$ are contained in $\Pi_{G_{v}^{a b}, \mathbf{q}}(u)$.

Notice that $\mathbf{p}_{1}(v b)=\mathbf{q}(a b) \subset \Pi_{G_{v}^{a b}, \mathbf{q}}(b)$ and hence all the hinges $\mathbf{p}_{1}(e)$ for $e \in$ $\delta_{G}(b)$ are contained in $\Pi_{G_{v}^{a b}, \mathbf{q}}(b)$. Similarly, $\mathbf{p}_{1}(v a)=L \subset \Pi_{G_{v}^{a b}, \mathbf{q}}(a)$ implies that all the hinges $\mathbf{p}_{1}(e)$ for $e \in \delta_{G}(a)$ are contained in $\Pi_{G_{v}^{a b}, \mathbf{q}}(a)$.

Finally, as for the two hinges $\mathbf{p}_{1}(v a)$ and $\mathbf{p}_{1}(v b)$ for $\delta_{G}(v)=\{v a, v b\}, \mathbf{p}_{1}(v b)=$ $\mathbf{q}(a b) \subset \Pi_{G_{v}^{a b}, \mathbf{q}}(a)$ and $\mathbf{p}_{1}(v a)=L \subset \Pi_{G_{v}^{a b}, \mathbf{q}}(a)$ hold. Hence, all the hinges $\mathbf{p}_{1}(e)$ for $e \in \delta_{G}(v)$ are contained in $\Pi_{G_{v}^{a b}, \mathbf{q}}(a)$. Thus $\left(G, \mathbf{p}_{1}\right)$ is a panel-hinge realization.

Although $\Pi_{G, \mathbf{p}_{1}}(v)$ and $\Pi_{G, \mathbf{p}_{1}}(a)$ are parallel in $\left(G, \mathbf{p}_{1}\right)$, at the end of the proof we will convert $\left(G, \mathbf{p}_{1}\right)$ to a nonparallel panel-hinge realization by slightly rotating $\Pi_{G, \mathbf{p}_{1}}(v)$ without decreasing the rank of the rigidity matrix. The following is an important observation provided by the configuration $\mathbf{p}_{1}$ of (6.12): From $\mathbf{p}_{1}(e)=\mathbf{q}(e)$ 
for every $e \in E_{v}$,

$$
R\left(G, \mathbf{p}_{1} ; E_{v}, V \backslash\{v\}\right)=R\left(G_{v}^{a b}, \mathbf{q} ; E_{v}, V \backslash\{v\}\right)
$$

(i.e., the part of the framework $\left(G, \mathbf{p}_{1}\right)$ which is not related to $\{v a, v b, a b\}$ is exactly the same as that of $\left.\left(G_{v}^{a b}, \mathbf{q}\right)\right)$. Let us take a look at $R\left(G, \mathbf{p}_{1}\right)$ :

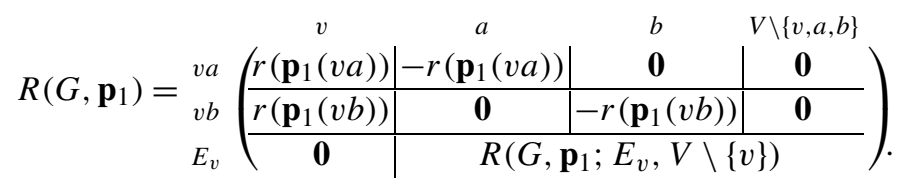

We shall perform fundamental column operations on $R\left(G, \mathbf{p}_{1}\right)$ which add the $j$ th column of $R\left(G, \mathbf{p}_{1} ; v\right)$ to that of $R\left(G, \mathbf{p}_{1} ; a\right)$ for each $1 \leq j \leq D$ one by one, i.e., $R\left(G, \mathbf{p}_{1}\right)$ is changed to

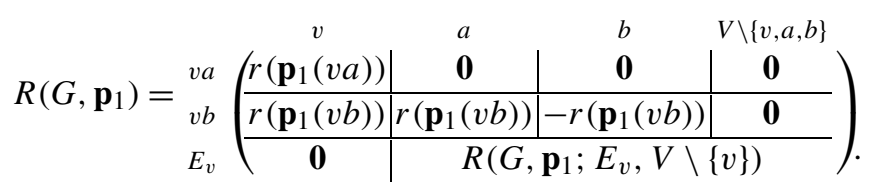

Substituting $\mathbf{p}_{1}(v b)=\mathbf{q}(a b)$ and (6.13) into (6.15), $R\left(G, \mathbf{p}_{1}\right)$ can be expressed by

$$
\begin{aligned}
R\left(G, \mathbf{p}_{1}\right)= & \begin{array}{c}
v a \\
v b \\
E_{v}
\end{array}\left(\begin{array}{c|c|c|c}
v & a & b & V \backslash\{v, a, b\} \\
\hline r\left(\mathbf{p}_{1}(v a)\right) & \mathbf{0} & \mathbf{0} & \mathbf{0} \\
\hline \mathbf{0} 1(v b)) & r(\mathbf{q}(a b)) & -r(\mathbf{q}(a b)) & \mathbf{0} \\
\hline & R\left(G_{v}^{a b}, \mathbf{q} ; E_{v}, V \backslash\{v\}\right)
\end{array}\right) \\
= & \begin{array}{c}
v a \\
v b \\
E_{v}
\end{array}\left(\begin{array}{c|c}
v\left(\mathbf{p}_{1}(v a)\right) & \mathbf{0} \\
\hline r\left(\mathbf{p}_{1}(v b)\right) & R\left(G_{v}^{a b}, \mathbf{q}\right)
\end{array}\right)
\end{aligned}
$$

where we used the fact that $R\left(G_{v}^{a b}, \mathbf{q}\right)$ is expressed by

$$
R\left(G_{v}^{a b}, \mathbf{q}\right)={ }_{E_{v}}^{a b}\left(\frac{r(\mathbf{q}(a b))|-r(\mathbf{q}(a b))|}{a} \mathbf{0}\right) .
$$

Therefore, from (6.11) and (6.16), we can show that $R\left(G, \mathbf{p}_{1}\right)$ has the desired rank, as follows:

$$
\begin{aligned}
\operatorname{rank} R\left(G, \mathbf{p}_{1}\right) & \geq \operatorname{rank} r\left(\mathbf{p}_{1}(v a)\right)+\operatorname{rank} R\left(G_{v}^{a b}, \mathbf{q}\right) \\
& =D-1+D(|V \backslash\{v\}|-1)-(k-1) \\
& =D(|V|-1)-k
\end{aligned}
$$

As we have remarked, $\left(G, \mathbf{p}_{1}\right)$ can be converted to a nonparallel realization without decreasing the rank of the rigidity matrix by Lemma 5.2. Thus, the statement follows. 


\section{$6.4 G$ is 2-edge-connected, Contains No Proper Rigid Subgraph, and $k=0$}

The remaining case is the most difficult one. Since the proof is quite complicated, we shall first show the 3-dimensional case, which must be most important. We then deal with the general dimensional case.

\subsubsection{3-Dimensional Case $(D=6)$}

Lemma 6.10 Let $G=(V, E)$ be a 2-edge-connected minimal 0-dof-graph with $|V| \geq 3$. Suppose that there exists no proper rigid subgraph in $G$ and that (6.1) holds. Then, there is a nonparallel panel-hinge realization $(G, \mathbf{p})$ in 3-dimensional space satisfying rank $R(G, \mathbf{p})=6(|V|-1)$.

Since the proof is lengthy, we first describe the proof outline.

Sketch of the proof Since $d=3$ and no proper rigid subgraph exists in $G$, Lemma 4.6 implies that there exist two vertices of degree two which are adjacent with each other. Let $v$ and $a$ be two such vertices and let $N_{G}(v)=\{a, b\}$ for some $b \in V$ and $N_{G}(a)=$ $\{v, c\}$ for some $c \in V$ (see Fig. 6(a)). Lemma 4.8 implies that both $G_{v}^{a b}$ and $G_{a}^{v c}$ are minimal 0-dof-graphs. Here $G_{a}^{v c}$ is the graph obtained by performing splitting off at $a$ along $v c$. By (6.1), there exist generic nonparallel panel-hinge realizations $\left(G_{v}^{a b}, \mathbf{q}\right)$ and $\left(G_{a}^{v c}, \mathbf{q}_{\rho}\right)$ where $\mathbf{q}_{\rho}$ will be defined later.

We shall first consider the realization $\left(G, \mathbf{p}_{1}\right)$ of $G$ based on $\left(G_{v}^{a b}, \mathbf{q}\right)$ which is exactly the same as the one we considered in Lemma 6.8 (see Fig. 6(d)). As in the proof of Lemma 6.8, we shall perform the row operations on $R\left(G, \mathbf{p}_{1}\right)$ so that $R\left(G_{v}^{a b}, \mathbf{q}\right)$ appears as a submatrix of $R\left(G, \mathbf{p}_{1}\right)$ (see Fig. 5). Let us remark the difference between $k=0$ (the current situation) and $k>0$ (Lemma 6.8). In the proof of Lemma 6.8 , we have proved that $\left(G, \mathbf{p}_{1}\right)$ attains the desired rank as $\operatorname{rank} R\left(G, \mathbf{p}_{1}\right) \geq 5+\operatorname{rank} R\left(G_{v}^{a b}, \mathbf{q}\right)=5+6(|V \backslash\{v\}|-1)-(k-1)=6(|V|-1)-k$, where rank $R\left(G_{v}^{a b}, \mathbf{q}\right)$ was equal to $6(|V \backslash\{v\}|-1)-(k-1)$ since $G_{v}^{a b}$ was a minimal $(k-1)$-dof-graph. In contrast, since rank $R\left(G_{v}^{a b}, \mathbf{q}\right)=6(|V \backslash\{v\}|-1)$, we only have $\operatorname{rank} R\left(G, \mathbf{p}_{1}\right) \geq 5+\operatorname{rank} R\left(G_{v}^{a b}, \mathbf{q}\right)=5+6(|V \backslash\{v\}|-1)=6(|V|-1)-1$ in the current situation, which does not complete the proof since we need to show rank $R\left(G, p_{1}\right)=6(|V|-1)$.

In order to show the existence of one more independent row, we use the fact that, in $\widetilde{G_{v}^{a b}}$, there exists at least one edge among $\widetilde{a b}$ which is not used in a base of the combinatorial matroid $\mathcal{M}\left(\widetilde{G_{v}^{a b}}\right)$ (Lemma 4.3). By using induction hypothesis, we can show that a corresponding redundant row exists in $R\left(G_{v}^{a b}, \mathbf{q}\right)$ among those associated with $\widetilde{a b}$. Let $(a b)_{i *}$ be the edge associated with such a redundant row. Then, this row can be converted to a zero vector by row operations in $R\left(G_{v}^{a b}, \mathbf{q}\right)$. We shall perform

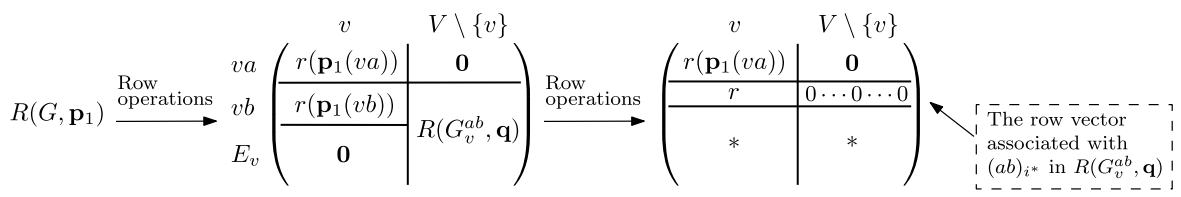

Fig. 5 Row operations 
these row operations on $R(G, \mathbf{p})$ by focusing on the fact that $R\left(G_{v}^{a b}, \mathbf{q}\right)$ is a submatrix of $R(G, \mathbf{p})$. Then, these row operations change $R(G, \mathbf{p})$ to the right-most matrix of Fig. 5 (assuming that $(a b)_{i^{*}}$ is the top row in $\left.R\left(G_{v}^{a b}, \mathbf{q}\right)\right)$. Our goal is to show that the top-left $6 \times 6$-submatrix of the right-most matrix is non-singular.

Unfortunately, there is a case where this $6 \times 6$-submatrix is singular no matter how we select $\mathbf{p}_{1}(v a)$. Contrary to the case of $k>0$, we need to construct two more panelhinge realizations $\left(G, \mathbf{p}_{2}\right)$ and $\left(G, \mathbf{p}_{3}\right)$. These two realizations are constructed based on the graphs $G_{v}^{a b}$ and $G_{a}^{v c}$, respectively. The realization $\left(G, \mathbf{p}_{2}\right)$ can be constructed in the manner symmetric to $\left(G, \mathbf{p}_{1}\right)$ by changing the role of $a$ and $b$ as shown in Fig. 6(e). (Although $\left(G, \mathbf{p}_{1}\right)$ and $\left(G, \mathbf{p}_{2}\right)$ are not nonparallel, we will convert them to nonparallel panel-hinge realizations by slightly rotating the panel of $v$ without decreasing the rank of the rigidity matrix by using Lemma 5.2.)

$\left(G, \mathbf{p}_{3}\right)$ is constructed as follows. It is not difficult to see that $G_{a}^{v c}$ is isomorphic to $G_{v}^{a b}$ (see Fig. 6(b) (f)) and hence there is the realization $\left(G_{a}^{v c}, \mathbf{q}_{\rho}\right)$ that represents the same panel-hinge framework as $\left(G_{v}^{a b}, \mathbf{q}\right)$ by regarding the panel of $a$ in $\left(G_{v}^{a b}, \mathbf{q}\right)$ as that of $v$ in $\left(G_{a}^{v c}, \mathbf{q}_{\rho}\right)$ as shown in Fig. 6(g). We shall then construct the realization $\left(G, \mathbf{p}_{3}\right)$ based on $\left(G_{a}^{v c}, \mathbf{q}_{\rho}\right)$ as shown in Fig. 6(h) (see (6.31) and (6.32) for the definitions of $\mathbf{q}_{\rho}$ and $\mathbf{p}_{3}$ ).

Let us briefly explain why at least one of $\left(G, \mathbf{p}_{1}\right),\left(G, \mathbf{p}_{2}\right)$ and $\left(G, \mathbf{p}_{3}\right)$ attains the desired rank with certain choices of $\mathbf{p}_{1}(v a), \mathbf{p}_{2}(v b), \mathbf{p}_{3}(a c)$. We first take a look at when the top-left $6 \times 6$-submatrix of $R\left(G, \mathbf{p}_{1}\right)$ given in the right-most matrix of Fig. 5 becomes singular. We focus on the following fact: (i) The last row vector, denoted $r$, of this $6 \times 6$-submatrix (see the right-most one of Fig. 5) is indeed a nonzero linear combination of $r_{i}\left(\mathbf{p}_{1}(v b)\right), i=1, \ldots, 5$ from the way of row operations. Moreover, from the definition of $\mathbf{p}_{1}$, we have $\mathbf{p}_{1}(v b)=\mathbf{q}(a b)$. Hence, $r$ is actually a linear combination of $r_{i}(\mathbf{q}(a b)), i=1, \ldots, 5$. (ii) From the definition of the rigidity matrix, if the top-left $6 \times 6$-submatrix is singular, $r$ is in the orthogonal complement of $\mathbf{p}_{1}(v a)$. Since we can take any line on the panel $\Pi_{G_{v}^{a b}, \mathbf{q}}(a)$ as $\mathbf{p}_{1}(v a)$, we found that $r$ is in the orthogonal complement of the vector space spanned by the extensors whose supporting lines lie on $\Pi_{G_{v}^{a b}, \mathbf{q}}(a)$.

We shall perform the same analysis on $\left(G, \mathbf{p}_{2}\right)$ and $\left(G, \mathbf{p}_{3}\right)$ : If the top-left $6 \times 6$ submatrix of $\left(G, \mathbf{p}_{2}\right)$ (obtained by the similar row operations as in Fig. 5), then (i') the last row vector $r^{\prime}$ of this $6 \times 6$-submatrix is a linear combination of $r_{i}(\mathbf{q}(a b)), i=$ $1, \ldots, 5$. Due to the symmetry of the construction, it can be observed that $r^{\prime}$ is indeed equal to $r$; (ii') if the top-left $6 \times 6$-submatrix is singular for any choice of $\mathbf{p}_{2}(v b)$, $r$ is in the orthogonal complement of the vector space spanned by the extensors whose supporting lines lie on the plane $\Pi_{G_{v}^{a b}, \mathbf{q}}(b)$.

If the top-left $6 \times 6$-submatrix of $\left(G, \mathbf{p}_{3}\right)$ (obtained by appropriate row operations), then $\left(\mathrm{i}^{\prime \prime}\right)$ the last row vector $r^{\prime \prime}$ of the corresponding $6 \times 6$-submatrix is a linear combination of $r_{i}(\mathbf{q}(a c)), i=1, \ldots, 5$. A key observation is that $r^{\prime \prime}$ is equal to $r$ (with the reversed sign) because $a$ is a degree two vertex in $G_{v}^{a b}$. (Roughly speaking, $r$ and $r^{\prime \prime}$ can be regarded as forces applied to the panel $\Pi_{G_{v}^{a b}, \mathbf{q}}(a)$ in $R\left(G_{v}^{a b}, \mathbf{q}\right)$ through the hinges $\mathbf{q}(a b)$ and $\mathbf{q}(a c)$, respectively. Since this panel is incident to only $\mathbf{q}(a b)$ and $\mathbf{q}(a c), r^{\prime \prime}$ must be equal to $-r$.) (ii') If the top-left $6 \times 6$-submatrix is singular for any choice of $\mathbf{p}_{3}(a c), r$ is in the orthogonal complement of the vector space spanned by the extensors whose supporting lines lie on the plane $\Pi_{G_{v}^{a b}, \mathbf{q}}(c)$. 
In total, if all these three $6 \times 6$-submatrices are singular, $r$ is in the orthogonal complement of the vector space spanned by the extensors whose supporting lines lie on $\Pi_{G_{v}^{a b}, \mathbf{q}}(a), \Pi_{G_{v}^{a b}, \mathbf{q}}(b)$, or $\Pi_{G_{v}^{a b}, \mathbf{q}}(c)$. However, if the configuration of these three panels is generic, then the set of extensors on them becomes 6-dimensional. This contradicts that $r$ is nonzero. Thus, we conclude that the top-left $6 \times 6$-submatrix is non-singular for at least one of $R\left(G, \mathbf{p}_{i}\right), i=1,2,3$, and this attains the desired rank, $6(|V|-1)$.

Let us start the rigorous proof.

Proof of Lemma 6.10 We use notation $v, a, b$, and $c$ defined in the sketch of the proof. As remarked in the proof of the previous lemma, $G$ is a simple graph. Also, by Lemma 6.7, we can assume that $|V| \geq 4$ and $G_{v}^{a b}$ is simple in the subsequent proof. Hence, by (6.1), there exists a generic nonparallel panel-hinge realization $\left(G_{v}^{a b}, \mathbf{q}\right)$ in $\mathbb{R}^{3}$, which satisfies

$$
\operatorname{rank} R\left(G_{v}^{a b}, \mathbf{q}\right)=6(|V \backslash\{v\}|-1) .
$$

Let $E_{v}=E \backslash\{v a, v b\}$. Let us recall that the mapping $\mathbf{p}_{1}$ on $E$ was defined by (6.12). We symmetrically define a mapping $\mathbf{p}_{2}$ on $E$ as follows:

$$
\mathbf{p}_{2}(e)= \begin{cases}\mathbf{q}(e) & \text { if } e \in E_{v} \\ \mathbf{q}(a b) & \text { if } e=v a \\ L^{\prime} & \text { if } e=v b\end{cases}
$$

where $L^{\prime}$ is a $(d-2)$-dimensional affine subspace contained in $\Pi_{G_{v}^{a b}, \mathbf{q}}(b)$. Then, the argument symmetric to Claim 6.9 implies that $\left(G, \mathbf{p}_{2}\right)$ is a panel-hinge realization of $G$. The frameworks $\left(G, \mathbf{p}_{1}\right)$ and $\left(G, \mathbf{p}_{2}\right)$ are depicted in Fig. 6(d) and (e), respectively.

Putting aside for a while the discussion concerning how $R\left(G, \mathbf{p}_{3}\right)$ is defined, we shall first investigate when $R\left(G, \mathbf{p}_{1}\right)$ or $R\left(G, \mathbf{p}_{2}\right)$ takes the desired rank (for some choice of $L \subset \Pi_{G_{v}^{a b}, \mathbf{q}}(a)$ or $\left.L^{\prime} \subset \Pi_{G_{v}^{a b}, \mathbf{q}}(b)\right) . R\left(G, \mathbf{p}_{1}\right)$ was described by (6.16), and $R\left(G, \mathbf{p}_{2}\right)$ is given as follows:

$$
R\left(G, \mathbf{p}_{2}\right)=\begin{gathered}
v b \\
v a \\
E_{v}
\end{gathered}\left(\begin{array}{c|c|c|c}
v & a & b & V \backslash\{v, a, b\} \\
\hline r\left(\mathbf{p}_{2}(v b)\right) & \mathbf{0} & -r\left(\mathbf{p}_{2}(v b)\right) & \mathbf{0} \\
\hline \mathbf{0} & \multicolumn{1}{c}{R\left(G, \mathbf{p}_{2} ; E_{v}, V \backslash\{v\}\right)}
\end{array}\right) .
$$

In a manner similar to the case of $R\left(G, \mathbf{p}_{1}\right)$, we perform the fundamental column operations on $R\left(G, \mathbf{p}_{2}\right)$ which add the $j$ th column of $R\left(G, \mathbf{p}_{2} ; v\right)$ to that of $R\left(G, \mathbf{p}_{2} ; b\right)$ for each $1 \leq j \leq 6$. Substituting $\mathbf{p}_{2}(v a)=\mathbf{q}(a b)$ and $R\left(G, \mathbf{p}_{2} ; E_{v}, V \backslash\right.$ $\{v\})=R\left(G_{v}^{a b}, \mathbf{q} ; E_{v}, V \backslash\{v\}\right)$ into the resulting matrix, we obtain

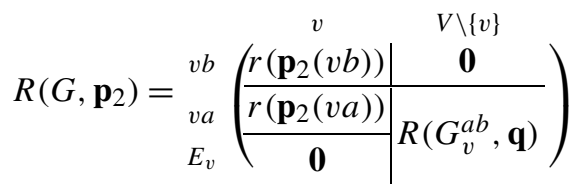

(see the proof of Lemma 6.8 for more details). 


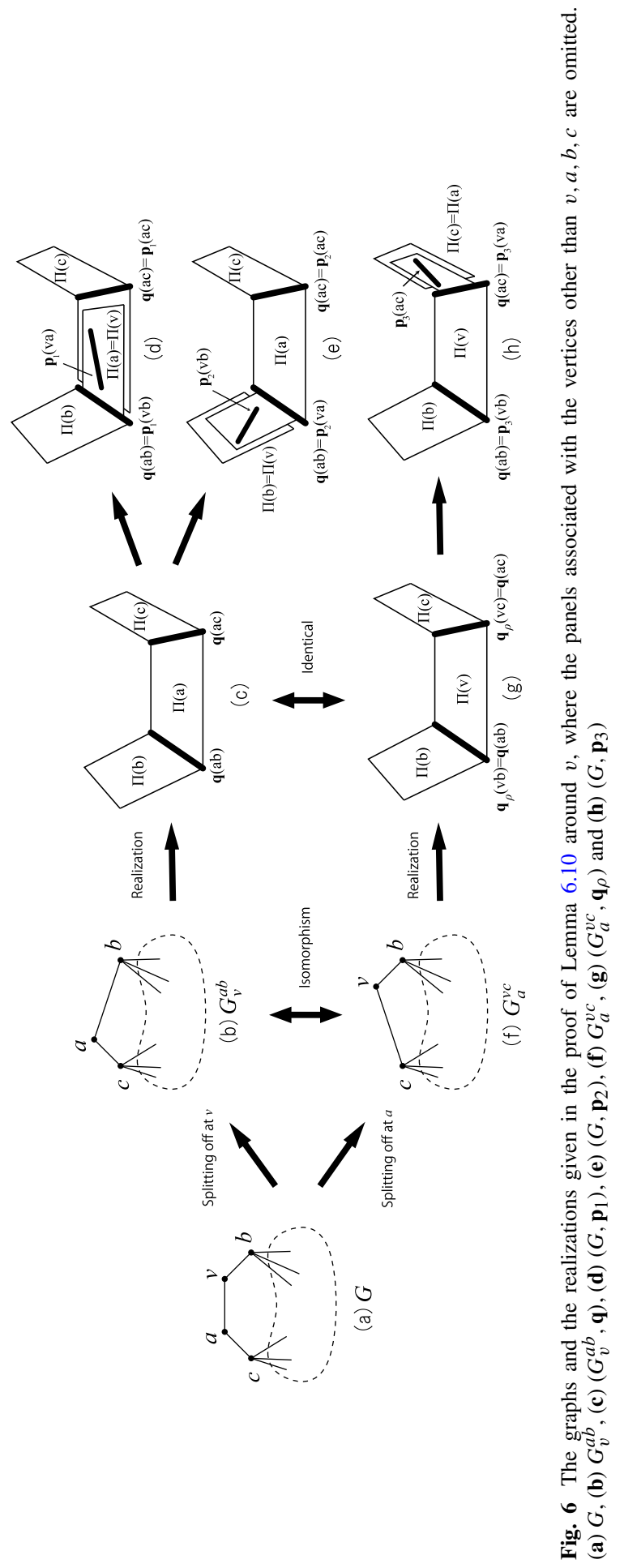


As discussed in the sketch of the proof, we shall further convert $R\left(G, \mathbf{p}_{1}\right)$ of (6.16) to the matrix given in (6.29) by applying appropriate fundamental row operations based on the claim below (Claim 6.11). The matrix given by (6.29) has the following two properties; (i) the entries of the submatrix $R\left(G, \mathbf{p}_{1} ;(v b)_{i^{*}}, V \backslash\{v\}\right.$ ) (whose size is $1 \times 6|V \backslash\{v\}|)$ are all zero, and (ii) the bottom-right submatrix (denoted by $R\left(G_{v}^{a b} \backslash\right.$ $\left.(a b)_{i^{*}}, \mathbf{q}\right)$ in (6.29)) has rank equal to $6(|V \backslash\{v\}|-1)$. This implies that, if the top-left $6 \times 6$-submatrix of (6.29) has full rank, then $\operatorname{rank} R\left(G, \mathbf{p}_{1}\right)=6+6(|V \backslash\{v\}|-1)=$ $6(|V|-1)$ holds. Symmetrically, we shall convert $R\left(G, \mathbf{p}_{2}\right)$ to that given in (6.30) which has the properties similar to (i) and (ii).

Let us first show how $R\left(G, \mathbf{p}_{1}\right)$ given in (6.16) is converted to (6.29). For this, let us focus on $R\left(G_{v}^{a b}, \mathbf{q}\right)$ given in (6.17) for a while. We say that a row vector of $R\left(G_{v}^{a b}, \mathbf{q}\right)$ is redundant if removing it from $R\left(G_{v}^{a b}, \mathbf{q}\right)$ does not decrease the rank of the matrix. The following claim is a key observation, which states a relation between the combinatorial matroid $\mathcal{M}\left(\widetilde{G_{v}^{a b}}\right)$ (i.e., the union of 6 graphic matroids) and the linear matroid derived from the row dependence of $R\left(G_{v}^{a b}, \mathbf{q}\right)$.

Claim 6.11 In $R\left(G_{v}^{a b}, \mathbf{q}\right)$, there exists a redundant row vector among those associated with $a b$.

Proof Recall a combinatorial property of $G_{v}^{a b}$ given in Lemma 4.3: Since $G_{v}^{a b}$ is a minimal $k$-dof-graph (with $k=0$ ), Lemma 4.3(ii) says that there exists a base $B^{\prime}$ of the matroid $\mathcal{M}\left(\widetilde{G_{v}^{a b}}\right)$ satisfying $\left|B^{\prime} \cap \widetilde{a b}\right|<5$. This implies that $\widetilde{G_{v}^{a b}}$ has some redundant edge $(a b)_{i}$ among $\widetilde{a b}$ with respect to $\mathcal{M}\left(\widetilde{G_{v}^{a b}}\right)$ (i.e., removing $(a b)_{i}$ from $\widetilde{G_{v}^{a b}}$ does not decrease the rank of $\left.\mathcal{M}\left(\widetilde{G_{v}^{a b}}\right)\right)$. We show that this redundancy also appears in the linear matroid derived from $R\left(G_{v}^{a b}, \mathbf{q}\right)$.

Let $B^{\prime}$ be a base of the matroid $\mathcal{M}\left(\widetilde{G_{v}^{a b}}\right)$ satisfying $\left|B^{\prime} \cap \widetilde{a b}\right|<5$ mentioned above. Let $h=\left|B^{\prime} \cap \tilde{a b}\right|$. Then, we have $h \leq 4$ and $\left|B^{\prime}\right|=6\left(|V \backslash\{v\}|-1\right.$ ) (since $G_{v}^{a b}$ is a 0 -dof-graph). We shall consider the graph $G_{v}$ that is obtained from $G_{v}^{a b}$ by removing $a b$. Clearly, $B^{\prime} \backslash \widetilde{a b}$ is an independent set of $\mathcal{M}\left(\widetilde{G_{v}}\right)$ with cardinality $6(|V \backslash\{v\}|-$ $1)-h$ and hence we have $\operatorname{def}\left(\widetilde{G_{v}}\right) \leq h$ by (2.4). Namely, $G_{v}$ is a $k^{\prime}$-dof-graph for some nonnegative integer $k^{\prime}$ with $k^{\prime} \leq h \leq 4$. Also, $G_{v}$ is minimal by Lemma 3.3. Let $\mathbf{q}_{E_{v}}$ denote the restriction of $\mathbf{q}$ to the edge set $E_{v}$ of $G_{v}$. We claim the following.

$$
\operatorname{rank} R\left(G_{v}, \mathbf{q}_{E_{v}}\right)=6(|V \backslash\{v\}|-1)-k^{\prime} \geq 6(|V \backslash\{v\}|-1)-4 .
$$

To see this, recall that, by induction hypothesis (6.1), the rigidity matrix of any generic nonparallel panel-hinge realization of $G_{v}$ takes rank equal to $6(|V \backslash\{v\}|-$ $1)-k^{\prime}{ }^{6}$ Recall also that $\left(G_{v}^{a b}, \mathbf{q}\right)$ was defined as a generic nonparallel realization of $G_{v}^{a b}$ in the inductive step. Hence, $\mathbf{q}$ was taken in such a way that the set of all the coefficients appearing in the equations expressing the panels is algebraically independent over $\mathbb{Q}$. This property clearly remains in the realization restricted to $E_{v}$ and

\footnotetext{
${ }^{6}$ If one particular nonparallel realization achieves rank equal to $6(|V \backslash\{v\}|-1)-k^{\prime}$, then all generic nonparallel realizations attain the same rank by the definition.
} 
hence $\left(G_{v}, \mathbf{q}_{E_{v}}\right)$ is also a generic nonparallel panel-hinge realization of $G_{v}$. Thus, (6.22) holds.

Note that $R\left(G_{v}, \mathbf{q}_{E_{v}}\right)$ is the matrix obtained from $R\left(G_{v}^{a b}, \mathbf{q}\right)$ by removing the 5 rows associated with $a b$. Note also that $R\left(G_{v}^{a b}, \mathbf{q}\right)$ has rank equal to $6(|V \backslash\{v\}|-1)$ by (6.18). Hence, from (6.22), adding at most 4 row vectors associated with $a b$ to the rows of $R\left(G_{v}, \mathbf{q}_{E_{v}}\right)$, we obtain a set of row vectors which spans the row space of $R\left(G_{v}^{a b}, \mathbf{q}\right)$. This implies that at least one row vector associated with $a b$ is redundant.

Let $i^{*}$ be the index of a redundant row associated with $a b$ shown in Claim 6.11. Namely, denoting by $R\left(G_{v}^{a b} \backslash(a b)_{i^{*}}, \mathbf{q}\right)$ the matrix obtained from $R\left(G_{v}^{a b}, \mathbf{q}\right)$ by removing the row associated with $(a b)_{i *}$, we have

$$
\operatorname{rank} R\left(G_{v}^{a b} \backslash(a b)_{i^{*}}, \mathbf{q}\right)=\operatorname{rank} R\left(G_{v}^{a b}, \mathbf{q}\right)=6(|V \backslash\{v\}|-1) .
$$

Also, since $R\left(G_{v}^{a b}, \mathbf{q} ;(a b)_{i^{*}}\right)$ is redundant, it can be expressed as a linear combination of the other row vectors of $R\left(G_{v}^{a b}, \mathbf{q}\right)$. Thus, there exists a scalar $\lambda_{e_{j}}$ for each $e_{j} \in \widetilde{E_{v}} \cup \widetilde{a b}$ such that

$$
\sum_{1 \leq j \leq 5} \lambda_{(a b)_{j}} R\left(G_{v}^{a b}, \mathbf{q} ;(a b)_{j}\right)+\sum_{e \in E_{v}, 1 \leq j \leq 5} \lambda_{e_{j}} R\left(G_{v}^{a b}, \mathbf{q} ; e_{j}\right)=\mathbf{0}
$$

where

$$
\lambda_{(a b)_{i *}}=1 .
$$

In other words, fundamental row operations change the matrix $R\left(G_{v}^{a b}, \mathbf{q}\right)$ so that the row associated with $(a b)_{i *}$ becomes a zero vector. Such row operations can be extended to $R\left(G, \mathbf{p}_{1}\right)$ of $(6.16)$, by focusing on the fact that $R\left(G, \mathbf{p}_{1} ; E \backslash\{v b\}, V \backslash\right.$ $\{v\})$ is equal to $R\left(G_{v}^{a b}, \mathbf{q}\right)$, so that the part of a row vector of $R\left(G, \mathbf{p}_{1}\right)$ corresponding to $V \backslash\{v\}$ becomes zero as in Fig. 5 .

Let us show that the resulting matrix can be described as (6.29) (up to some row exchanges). To show this, let us take a look at the row operations more precisely. Since the row associated with $(a b)_{i *}$ in $R\left(G_{v}^{a b}, \mathbf{q}\right)$ corresponds to that associated with $(v b)_{i^{*}}$ in $R\left(G, \mathbf{p}_{1}\right)$ (see (6.16)), performing such row operations is equivalent to the addition of

$$
\sum_{1 \leq j \leq 5, j \neq i^{*}} \lambda_{(a b)_{j}} R\left(G, \mathbf{p}_{1} ;(v b)_{j}\right)+\sum_{e \in E_{v}, 1 \leq j \leq 5} \lambda_{e_{j}} R\left(G, \mathbf{p}_{1} ; e_{j}\right)
$$

to the row associated with $(v b)_{i^{*}}$ in (6.16). Namely, the row operations convert $R\left(G, \mathbf{p}_{1} ;(v b)_{i^{*}}\right)$ of (6.16) to

$$
\sum_{1 \leq j \leq 5} \lambda_{(a b)_{j}} R\left(G, \mathbf{p}_{1} ;(v b)_{j}\right)+\sum_{e \in E_{v}, 1 \leq j \leq 5} \lambda_{e_{j}} R\left(G, \mathbf{p}_{1} ; e_{j}\right)
$$

where $\lambda_{(a b)_{i} *}=1$. (Compare it with the left hand side of (6.24).) From $R\left(G, \mathbf{p}_{1} ; E \backslash\right.$ $\{v a\}, V \backslash\{v\})=R\left(G_{v}^{a b}, \mathbf{q}\right)$ and from (6.24), the components of (6.27) associated 
with $V \backslash\{v\}$ become all zero. As for the remaining part of the new row vector (6.27) (i.e., the components associated with $v$ ), since $R\left(G, \mathbf{p}_{1} ; E_{v}, v\right)=\mathbf{0}$ holds as shown in (6.16), we have

$$
\begin{aligned}
& \sum_{1 \leq j \leq 5} \lambda_{(a b)_{j}} R\left(G, \mathbf{p}_{1} ;(v b)_{j}, v\right)+\sum_{e \in E_{v}, 1 \leq j \leq 5} \lambda_{e_{j}} R\left(G, \mathbf{p}_{1} ; e_{j}, v\right) \\
& \quad=\sum_{1 \leq j \leq 5} \lambda_{(a b)_{j}} R\left(G, \mathbf{p}_{1} ;(v b)_{j}, v\right) .
\end{aligned}
$$

Note that $R\left(G, \mathbf{p}_{1} ;(v b)_{j}, v\right)=r_{j}\left(\mathbf{p}_{1}(v b)\right)=r_{j}(\mathbf{q}(a b))$ by the definition of the rigidity matrix and by $\mathbf{p}_{1}(v b)=\mathbf{q}(a b)$. Thus, we have seen that the new row vector (6.27) can be written as

$$
\left(\sum_{j} \lambda_{(a b)_{j}}^{v} r_{j}(\mathbf{q}(a b)), \quad \stackrel{V \backslash\{v\}}{\mathbf{0}}\right)
$$

As a result, the fundamental row operations change $R\left(G, \mathbf{p}_{1}\right)$ of (6.16) to the matrix described as (up to some row exchange of $(v b)_{i^{*}}$ )

$$
R\left(G, \mathbf{p}_{1}\right)={ }_{(v b)_{i^{*}}}^{v a}\left(\begin{array}{c|c}
v & V \backslash\{v\} \\
\hline \sum_{j} \lambda_{(a b)_{j}} r_{j}(\mathbf{q}(a b)) & \mathbf{0} \\
\hline * & R\left(G_{v}^{a b} \backslash(a b)_{i^{*}}, \mathbf{q}\right)
\end{array}\right) .
$$

Applying the symmetric argument to $R\left(G, \mathbf{p}_{2}\right)$ shown in $(6.21)$, we also have

$$
R\left(G, \mathbf{p}_{2}\right)={ }_{(v a)_{i} *}^{v b}\left(\begin{array}{c|c}
v & V \backslash\{v\} \\
\hline \sum_{j} \lambda_{(a b)_{j}} r_{j}(\mathbf{q}(a b)) & \mathbf{0} \\
\hline * & R\left(G_{v}^{a b} \backslash(a b)_{i}, \mathbf{q}\right)
\end{array}\right) .
$$

Note that the same $\lambda_{(a b)_{j}}, 1 \leq j \leq 5$ and the index $i^{*}$ are used in (6.29) and (6.30) since they are determined by $\left(G_{v}^{a b}, \mathbf{q}\right)$ and are independent of $\mathbf{p}_{1}$ and $\mathbf{p}_{2}$. It is not difficult to see that, if the top-left $6 \times 6$-submatrix of (6.29) has full rank, then we obtain $\operatorname{rank} R\left(G, \mathbf{p}_{1}\right) \geq 6+\operatorname{rank} R\left(G_{v}^{a b} \backslash(a b)_{i^{*}}, \mathbf{q}\right)=6(|V|-1)$ by (6.29) and (6.23), and we are done. Symmetrically, if the top-left $6 \times 6$-submatrix of (6.30) has full rank, then $R\left(G, \mathbf{p}_{2}\right)$ attains the desired rank. In $d=2$ we can show that the corresponding top-left submatrix has full rank in at least one of (6.29) and (6.30), but this may not be true for $d \geq 3$. Hence, we shall introduce another framework $\left(G, \mathbf{p}_{3}\right)$ in the following discussion.

Let us consider splitting off at $a$ along $v c$. (Recall that $a$ is a vertex of degree two.) Then, since $v$ and $a$ are adjacent vertices of degree two, it is not difficult to see that the resulting graph $G_{a}^{v c}$ is isomorphic to $G_{v}^{a b}$ (see Fig. 6(b) and (f)) by the isomorphism $\rho: V \backslash\{a\} \rightarrow V \backslash\{v\}$, i.e., $\rho(v)=a$ and $\rho(u)=u$ for $u \in V \backslash\{v, a\}$. The isomorphism $\rho$ induces the mapping $\mathbf{q}_{\rho}$ on $E \backslash\{v a, a c\} \cup\{v c\}$ in a natural way 
defined by, for $e=u w \in E \backslash\{v a, a c\} \cup\{v c\}$,

$$
\mathbf{q}_{\rho}(e)=\mathbf{q}(\rho(u) \rho(w))= \begin{cases}\mathbf{q}(a b) & \text { if } e=v b \\ \mathbf{q}(a c) & \text { if } e=v c \\ \mathbf{q}(e) & \text { otherwise }\end{cases}
$$

The isomorphism $\rho$ between $G_{v}^{a b}$ and $G_{a}^{v c}$ implies that $\left(G_{a}^{v c}, \mathbf{q}_{\rho}\right)$ and $\left(G_{v}^{a b}, \mathbf{q}\right)$ represent the same panel-hinge framework in $\mathbb{R}^{3}$. In particular, we have $\Pi_{G_{a}^{v c}, \mathbf{q}_{\rho}}(u)=$ $\Pi_{G_{v}^{a b}, \mathbf{q}}(u)$ for each $u \in V \backslash\{v, a\}$ and $\Pi_{G_{a}^{v c}, \mathbf{q}_{\rho}}(v)=\Pi_{G_{v}^{a b}, \mathbf{q}}(a)$.

We shall construct a similar extension of the mapping $\mathbf{q}_{\rho}$ as was done in $\mathbf{p}_{1}$ or $\mathbf{p}_{2}$. Define a mapping $\mathbf{p}_{3}$ on $E$ as

$$
\mathbf{p}_{3}(e)= \begin{cases}\mathbf{q}_{\rho}(e) & \text { if } e \in E \backslash\{v a, a c\} \\ L^{\prime \prime} & \text { if } e=a c \\ \mathbf{q}_{\rho}(v c) & \text { if } e=v a\end{cases}
$$

where $L^{\prime \prime}$ is a $(d-2)$-dimensional affine subspace contained in $\Pi_{G_{a}^{v c}, \mathbf{q}_{\rho}}(c)(=$ $\left.\Pi_{G_{v}^{a b}, \mathbf{q}}(c)\right)$ and it can be shown as in Claim 6.9 that $\left(G, \mathbf{p}_{3}\right)$ is a panel-hinge realization of $G$ in $\mathbb{R}^{3}$ for any choice of $L^{\prime \prime} \subset \Pi_{G_{v}^{a b}, \mathbf{q}}(c)$ (see Fig. 6(h)). Combining (6.31) and (6.32), we actually have

$$
\mathbf{p}_{3}(e)= \begin{cases}\mathbf{q}(a c) & \text { if } e=v a \\ \mathbf{q}(a b) & \text { if } e=v b \\ L^{\prime \prime} & \text { if } e=a c \\ \mathbf{q}(e) & \text { otherwise. }\end{cases}
$$

Therefore, we have

$$
\begin{aligned}
r\left(\mathbf{p}_{3}(v a)\right) & =r(\mathbf{q}(a c)) \\
r\left(\mathbf{p}_{3}(v b)\right) & =r(\mathbf{q}(a b)) \\
R\left(G, \mathbf{p}_{3} ; E \backslash\{v a, v b, a c\}, V \backslash\{v, a\}\right) & =R\left(G_{v}^{a b}, \mathbf{q} ; E \backslash\{v a, v b, a c\}, V \backslash\{v, a\}\right) .
\end{aligned}
$$

We are now going to convert the rigidity matrix $R\left(G, \mathbf{p}_{3}\right)$ to that given in (6.41), which is an analogous form to (6.29) or (6.30). Let us take a look at $R\left(G, \mathbf{p}_{3}\right)$ :

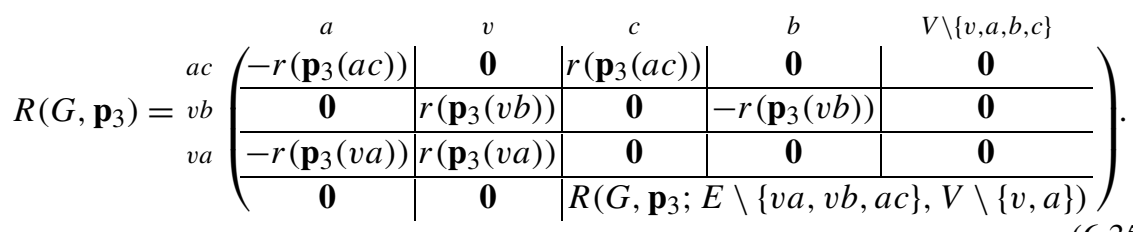

Consider the fundamental column operations which add the $j$ th column of $R\left(G, \mathbf{p}_{3} ; a\right)$ to that of $R\left(G, \mathbf{p}_{3} ; c\right)$ for each $1 \leq j \leq 6$. Then $R\left(G, \mathbf{p}_{3}\right)$ results in the following ma- 
trix:

$$
R\left(G, \mathbf{p}_{3}\right)=\quad \begin{array}{r|c|c|c|c|c}
a & v & c & b & V \backslash\{v, a, b, c\} \\
v b & v a
\end{array}\left(\begin{array}{c|c|c|c|c}
-r\left(\mathbf{p}_{3}(a c)\right) & \mathbf{0} & \mathbf{0} & \mathbf{0} & \mathbf{0} \\
\hline \mathbf{0} & r\left(\mathbf{p}_{3}(v b)\right) & \mathbf{0} & -r\left(\mathbf{p}_{3}(v b)\right) & \mathbf{0} \\
\hline-r\left(\mathbf{p}_{3}(v a)\right) & r\left(\mathbf{p}_{3}(v a)\right) & -r\left(\mathbf{p}_{3}(v a)\right) & \mathbf{0} & \mathbf{0} \\
\hline \mathbf{0} & \mathbf{0} & \mid R\left(G, \mathbf{p}_{3} ; E \backslash\{v a, v b, a c\}, V \backslash\{v, a\}\right)
\end{array}\right) .
$$

Substituting (6.34) into (6.36), $R\left(G, \mathbf{p}_{3}\right)$ becomes

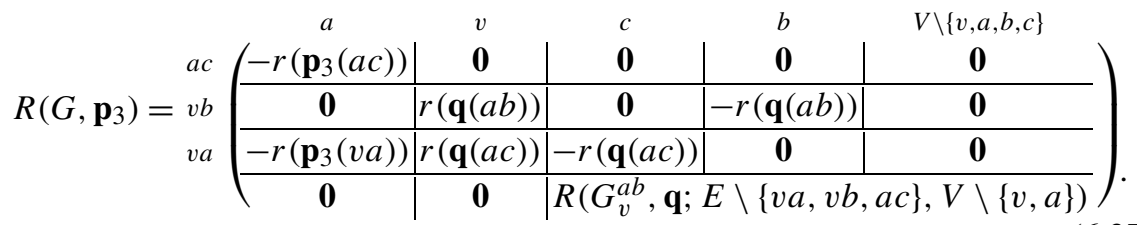

Note that $R\left(G_{v}^{a b}, \mathbf{q}\right)$ can be described by (up to some row exchanges)

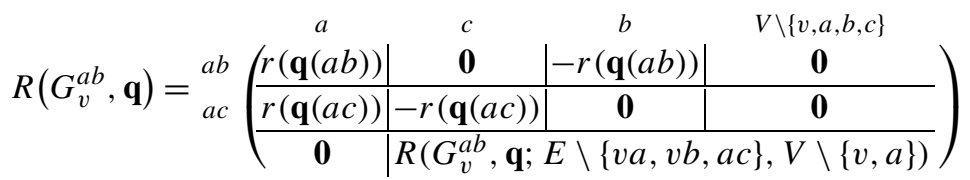

which is equal to $R\left(G, \mathbf{p}_{3} ; E \backslash\{a c\}, V \backslash\{a\}\right)$ given in (6.37). Therefore, (6.37) becomes

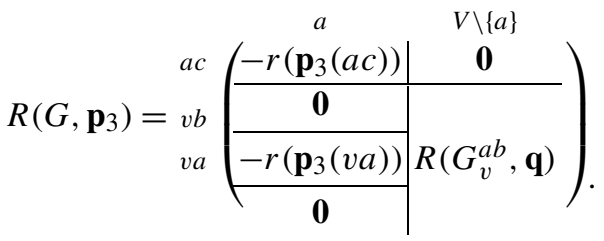

Recall that the row of $R\left(G_{v}^{a b}, \mathbf{q}\right)$ associated with $(a b)_{i *}$ is redundant, i.e., removing the row associated with $(a b)_{i^{*}}$ from $R\left(G_{v}^{a b}, \mathbf{q}\right)$ preserves the rank of $R\left(G_{v}^{a b}, \mathbf{q}\right)$ as shown in (6.23). In order to indicate the dependence of the row vectors within $R\left(G_{v}^{a b}, \mathbf{q}\right)$, we have introduced $\lambda_{e_{j}} \in \mathbb{R}$ for each $e_{j} \in \widetilde{E_{v}} \cup \widetilde{a b}$ so that they satisfy (6.24).

We shall again consider the row operations which convert $R\left(G_{v}^{a b}, \mathbf{q} ;(a b)_{i^{*}}\right)$ to a zero vector within $R\left(G_{v}^{a b}, \mathbf{q}\right)$ and apply these row operations to the matrix $R\left(G, \mathbf{p}_{3}\right)$ of (6.39) as shown in Fig. 7.

Note that, within the equation $R\left(G, \mathbf{p}_{3} ; E \backslash\{a c\}, V \backslash\{a\}\right)=R\left(G_{v}^{a b}, \mathbf{q}\right)$ of (6.39), we have the following row correspondence:

$$
\begin{aligned}
& R\left(G, \mathbf{p}_{3} ; v b, V \backslash\{a\}\right)=R\left(G_{v}^{a b}, \mathbf{q} ; a b\right) \\
& R\left(G, \mathbf{p}_{3} ; v a, V \backslash\{a\}\right)=R\left(G_{v}^{a b}, \mathbf{q} ; a c\right) \\
& R\left(G, \mathbf{p}_{3} ; e, V \backslash\{a\}\right)=R\left(G_{v}^{a b}, \mathbf{q} ; e\right) \text { for each } e \in E \backslash\{v a, v b, a c\} .
\end{aligned}
$$




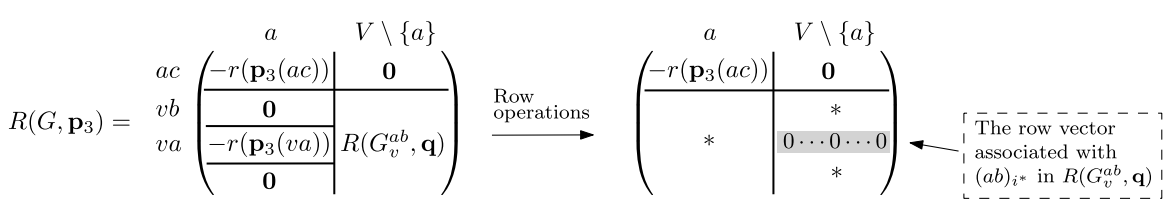

Fig. 7 Row operations on $R\left(G, \mathbf{p}_{3}\right)$

In particular, the row of $(a b)_{i^{*}}$ in $R\left(G_{v}^{a b}, \mathbf{q}\right)$ is corresponding to the row of $(v b)_{i^{*}}$ in $R\left(G, \mathbf{p}_{3}\right)$. Therefore, we have performed the fundamental row operations which add

$$
\begin{aligned}
& \sum_{1 \leq j \leq 5, j \neq i^{*}} \lambda_{(a b)_{j}} R\left(G, \mathbf{p}_{3} ;(v b)_{j}\right)+\sum_{1 \leq j \leq 5} \lambda_{(a c)_{j}} R\left(G, \mathbf{p}_{3} ;(v a)_{j}\right) \\
& +\sum_{\substack{e \in E \backslash\{v a, v b, a c\} \\
1 \leq j \leq 5}} \lambda_{e_{j}} R\left(G, \mathbf{p}_{3} ; e_{j}\right)
\end{aligned}
$$

to $R\left(G, \mathbf{p}_{3} ;(v b)_{i^{*}}\right)$ of (6.39). Namely, by $\lambda_{(a b)_{i^{*}}}=1$, the resulting row becomes

$$
\begin{aligned}
& \sum_{1 \leq j \leq 5} \lambda_{(a b)_{j}} R\left(G, \mathbf{p}_{3} ;(v b)_{j}\right)+\sum_{1 \leq j \leq 5} \lambda_{(a c)_{j}} R\left(G, \mathbf{p}_{3} ;(v a)_{j}\right) \\
& +\sum_{\substack{e \in E \backslash\{v a, v b, a c\} \\
1 \leq j \leq 5}} \lambda_{e_{j}} R\left(G, \mathbf{p}_{3} ; e_{j}\right) .
\end{aligned}
$$

By using the row correspondence between $R\left(G, \mathbf{p}_{3}\right)$ and $R\left(G_{v}^{a b}, \mathbf{q}\right)$ and also by using (6.24), the new row vector (6.40) can be reduced to, (by the same analysis as in the case of $\left.R\left(G, \mathbf{p}_{1}\right)\right)$,

$$
\left(-\sum_{j} \lambda_{(a c)_{j}}^{a} r_{j}\left(\mathbf{p}_{3}(v a)\right), \quad \stackrel{V \backslash\{a\}}{\mathbf{0}}\right) .
$$

Notice that, since the row of $(v a)_{j}$ in $R\left(G, \mathbf{p}_{3}\right)$ is corresponding to the row of $(a c)_{j}$ in $R\left(G_{v}^{a b}, \mathbf{p}_{3}\right)$, the coefficient of $r_{j}\left(\mathbf{p}_{3}(v a)\right)$ is indexed by $(a c)_{j}$. Moreover, since $\mathbf{p}_{3}(v a)=\mathbf{q}(a c)$ by $(6.34)$, this becomes

$$
\left(-\sum_{j} \lambda_{(a c)_{j}}^{a} r_{j}(\mathbf{q}(a c)), \quad \stackrel{V \backslash\{a\}}{\mathbf{0}}\right) .
$$

As a result, $R\left(G, \mathbf{p}_{3}\right)$ of (6.39) is changed to the following matrix by the row operations:

$$
R\left(G, \mathbf{p}_{3}\right)={ }_{(v b)_{i} *}^{a c}\left(\begin{array}{c|c}
a & V \backslash\{a\} \\
\hline \sum_{j} \lambda_{(a c)_{j}} r_{j}(\mathbf{q}(a c)) & \mathbf{0} \\
\hline * & R\left(G_{v}^{a b} \backslash(a b)_{i^{*}}, \mathbf{q}\right)
\end{array}\right)
$$

where $R\left(G_{v}^{a b} \backslash(a b)_{i^{*}}, \mathbf{q}\right)$ was defined as the matrix obtained from $R\left(G_{v}^{a b}, \mathbf{q}\right)$ by removing the row associated with $(a b)_{i}$ and satisfies (6.23). 
We finally show in the following Claim 6.12 that at least one of the top-left $6 \times 6$ submatrices of (6.29), (6.30), and (6.41) has full rank. Let us simply denote these three submatrices by $M_{1}, M_{2}$ and $M_{3}$, respectively, i.e.,

$$
\begin{aligned}
& M_{1}=\left(\begin{array}{c}
r\left(\mathbf{p}_{1}(v a)\right) \\
\sum_{j} \lambda_{(a b)_{j}} r_{j}(\mathbf{q}(a b))
\end{array}\right) \\
& M_{2}=\left(\begin{array}{c}
r\left(\mathbf{p}_{2}(v b)\right) \\
\sum_{j} \lambda_{(a b)_{j}} r_{j}(\mathbf{q}(a b))
\end{array}\right) \\
& M_{3}=\left(\begin{array}{c}
r\left(\mathbf{p}_{3}(a c)\right) \\
\sum_{j} \lambda_{(a c)_{j}} r_{j}(\mathbf{q}(a c))
\end{array}\right) .
\end{aligned}
$$

According to definitions (6.12), (6.19) and (6.32), we denote $\mathbf{p}_{1}(v a)=L, \mathbf{p}_{2}(v b)=$ $L^{\prime}$, and $\mathbf{p}_{3}(a c)=L^{\prime \prime}$, where we may choose any $(d-2)$-dimensional affine subspaces $L \subset \Pi_{G_{v}^{a b}, \mathbf{q}}(a), L^{\prime} \subset \Pi_{G_{v}^{a b}, \mathbf{q}}(b)$, and $L^{\prime \prime} \subset \Pi_{G_{v}^{a b}, \mathbf{q}}(c)$, respectively. Hence, $M_{1}, M_{2}$, and $M_{3}$ are described as

$$
\begin{aligned}
M_{1} & =\left(\begin{array}{c}
r(L) \\
\sum_{j} \lambda_{(a b)_{j}} r_{j}(\mathbf{q}(a b))
\end{array}\right) \\
M_{2} & =\left(\begin{array}{c}
r\left(L^{\prime}\right) \\
\sum_{j} \lambda_{(a b)_{j}} r_{j}(\mathbf{q}(a b))
\end{array}\right) \\
M_{3} & =\left(\begin{array}{c}
r\left(L^{\prime \prime}\right) \\
\sum_{j} \lambda_{(a c)_{j}} r_{j}(\mathbf{q}(a c))
\end{array}\right) .
\end{aligned}
$$

The following Claim 6.12 implies that at least one of $R\left(G, \mathbf{p}_{1}\right), R\left(G, \mathbf{p}_{2}\right)$ and $R\left(G, \mathbf{p}_{3}\right)$ attains the desired rank $6(|V|-1)$.

Claim 6.12 At least one of $M_{1}, M_{2}$, and $M_{3}$ has full rank for some choices of $L \subset$ $\Pi_{G_{v}^{a b}, \mathbf{q}}(a), L^{\prime} \subset \Pi_{G_{v}^{a b}, \mathbf{q}}(b)$, and $L^{\prime \prime} \subset \Pi_{G_{v}^{a b}, \mathbf{q}}(c)$.

Proof Let $r \in \mathbb{R}^{6}$ be $\sum_{j} \lambda_{(a b)_{j}} r_{j}(\mathbf{q}(a b))$. Note that $r$ is a nonzero vector because $r_{1}(\mathbf{q}(a b)), \ldots, r_{5}(\mathbf{q}(a b))$ are linearly independent and also $\lambda_{(a b)_{i *}}=1$. Suppose that $M_{1}$ given in (6.42) does not have full rank. Then, $r$ is contained in the row space of $r(L)$. This is equivalent to that $r$ is contained in the orthogonal complement of the vector space spanned by a 2-extensor $C(L)$ supporting $L$. Similarly, if $M_{2}$ given in (6.42) does not have full rank, then $r$ is contained in the orthogonal complement of the vector space spanned by $C\left(L^{\prime}\right)$.

We claim the following: If $M_{3}$ does not have full rank, then $r$ is also contained in the orthogonal complement of the space spanned by $C\left(L^{\prime \prime}\right)$. To see this, we need to remind ourselves of (6.24) indicating the row dependence within $R\left(G_{v}^{a b}, \mathbf{q}\right)$. Focusing on the six consecutive components associated with $a$ in (6.24), we have

$$
\sum_{\substack{e \in E_{v} \cup\{a b\} \\ 1 \leq j \leq 5}} \lambda_{e_{j}} R\left(G_{v}^{a b}, \mathbf{q} ; e_{j}, a\right)=\mathbf{0} .
$$


Recall that $R\left(G_{v}^{a b}, \mathbf{q} ; e, a\right)=\mathbf{0}$ holds if $e$ is not incident to $a$ in $G_{v}^{a b}$ according to the definition of a rigidity matrix. Since only $a b$ and $a c$ are incident to $a$ in $G_{v}^{a b}$, we actually have

$$
\begin{aligned}
\mathbf{0} & =\sum_{\substack{e \in E_{v} \cup\{a b\} \\
1 \leq j \leq 5}} \lambda_{e_{j}} R\left(G_{v}^{a b}, \mathbf{q} ; e_{j}, a\right) \\
& =\sum_{1 \leq j \leq 5} \lambda_{(a b)_{j}} R\left(G_{v}^{a b}, \mathbf{q} ;(a b)_{j}, a\right)+\sum_{1 \leq j \leq 5} \lambda_{(a c)_{j}} R\left(G_{v}^{a b}, \mathbf{q} ;(a c)_{j}, a\right) \\
& =\sum_{1 \leq j \leq 5} \lambda_{(a b)_{j}} r_{j}(\mathbf{q}(a b))+\sum_{1 \leq j \leq 5} \lambda_{(a c)_{j}} r_{j}(\mathbf{q}(a c)) .
\end{aligned}
$$

This in turn implies

$$
r=-\sum_{1 \leq j \leq 5} \lambda_{(a c)_{j}} r_{j}(\mathbf{q}(a c))
$$

If $M_{3}$ does not have full rank, then $\sum_{j} \lambda_{(a c)} r_{j}(\mathbf{q}(a c))$ is contained in the orthogonal complement of the space spanned by $C\left(L^{\prime \prime}\right)$, which means that $r$ is contained in the orthogonal complement of the space spanned by $C\left(L^{\prime \prime}\right)$ as well, by (6.44).

As a result, we found that, if none of $M_{1}, M_{2}$ and $M_{3}$ has full rank, then there exists a nonzero vector $r \in \mathbb{R}^{6}$ contained in the orthogonal complement of the vector space spanned by the set of 2-extensors

$$
\left(\bigcup_{L \subset \Pi(a)} C(L)\right) \cup\left(\bigcup_{L^{\prime} \subset \Pi(b)} C\left(L^{\prime}\right)\right) \cup\left(\bigcup_{L^{\prime \prime} \subset \Pi(c)} C\left(L^{\prime \prime}\right)\right),
$$

where $\Pi(u)$ denotes $\Pi_{G_{v}^{a b}, \mathbf{q}}(u)$ for each $u \in\{a, b, c\}$. Therefore, in order to show that at least one of $M_{1}, M_{2}$ and $M_{3}$ has full rank, it is enough to show that the dimension of the vector space spanned by (6.45) is equal to 6 .

To show this, let us take four points in $\mathbb{R}^{3}$ as follows; $p_{1}=\Pi(a) \cap \Pi(b) \cap$ $\Pi(c), p_{2} \in \Pi(a) \cap \Pi(b) \backslash \Pi(c), p_{3} \in \Pi(b) \cap \Pi(c) \backslash \Pi(a)$, and $p_{4} \in \Pi(c) \cap \Pi(a) \backslash$ $\Pi(b)$. Since $\left(G_{v}^{a b}, \mathbf{q}\right)$ is a generic nonparallel framework, we can take such four points in such a way that they are affinely independent. Also, notice that any line connecting two points among $\left\{p_{1}, \ldots, p_{4}\right\}$ is contained in $\Pi(a) \cup \Pi(b) \cup \Pi(c)$, and hence any 2 -extensor obtained from two points among $\left\{p_{1}, \ldots, p_{4}\right\}$ belongs to (6.45). Recall that, by Lemma 2.1, the set of 2-extensors $\left\{\boldsymbol{p}_{i} \vee \boldsymbol{p}_{j} \mid 1 \leq i<j \leq 4\right\}$ is linearly independent, which means that the dimension of the vector space spanned by (6.45) is equal to $\left(\begin{array}{l}4 \\ 2\end{array}\right)=6$.

As a result, at least one of $\left(G, \mathbf{p}_{1}\right),\left(G, \mathbf{p}_{2}\right)$ and $\left(G, \mathbf{p}_{3}\right)$ attains rank equal to $6(|V|-1)$. Although $\left(G, \mathbf{p}_{1}\right),\left(G, \mathbf{p}_{2}\right)$ and $\left(G, \mathbf{p}_{3}\right)$ are not nonparallel, we can convert them to nonparallel panel-hinge realizations by slightly rotating the panel associated with $v$ (or that associated with $a$ ) without decreasing the rank of the rigidity matrix by Lemma 5.2. This completes the proof of Lemma 6.10. 


\subsubsection{General Dimension}

Finally let us describe the general dimensional version of Lemma 6.10 .

Lemma 6.13 Let $G=(V, E)$ be a 2-edge-connected minimal 0-dof-graph with $|V| \geq 3$. Suppose that there exists no proper rigid subgraph in $G$ and that (6.1) holds. Then, there is a nonparallel panel-hinge realization $(G, \mathbf{p})$ in $\mathbb{R}^{d}$ satisfying $\operatorname{rank} R(G, \mathbf{p})=D(|V|-1)$.

Proof By Lemma 4.6, either $G$ is a cycle of length at most $d$ or $G$ has a chain of length $d$. If $G$ is a cycle of length at most $d$, then we are done by Lemma 5.4. Hence, let us consider the case where $G$ has a chain $v_{0} v_{1} v_{2} \ldots v_{d}$ of length $d$ (where $d_{G}\left(v_{i}\right)=2$ for $\left.1 \leq i \leq d-1\right)$.

The proof strategy is exactly the same as $d=3$. In general case, we shall consider $d$ distinct frameworks based on the chain $v_{0} v_{1} v_{2} \ldots v_{d}$. For each $1 \leq i \leq d-1$, the graph $G_{v_{i}}^{v_{i-1} v_{i+1}}$ obtained by splitting off at $v_{i}$ is a minimal 0-dof-graph by Lemma 4.8. Let us simply denote $G_{v_{i}}^{v_{i-1} v_{i+1}}$ by $G_{i}$ for each $1 \leq i \leq d-1$. By (6.1), there exist nonparallel panel-hinge realizations $\left(G_{i}, \mathbf{q}_{i}\right)$ for $1 \leq i \leq d-1$ such that they represent the same framework in $\mathbb{R}^{d}$. Based on $\left(G_{i}, \mathbf{q}_{i}\right)$, we shall construct $d$ distinct frameworks for $G$ in the same way as in 3-dimensional case. We shall show that at least one of them attains the desired rank.

Let us start the proof. Consider $G_{1}=\left(V \backslash\left\{v_{1}\right\}, E \backslash\left\{v_{0} v_{1}, v_{1} v_{2}\right\} \cup\left\{v_{0} v_{2}\right\}\right)$. By (6.1), there exists a generic nonparallel panel-hinge framework $\left(G_{1}, \mathbf{q}_{1}\right)$ which satisfies

$$
R\left(G_{1}, \mathbf{q}_{1}\right)=D(|V|-2) .
$$

We first define two frameworks $\left(G, \mathbf{p}_{0}\right)$ and $\left(G, \mathbf{p}_{1}\right)$ based on $\left(G_{1}, \mathbf{q}_{1}\right)$; for $e \in E$,

$$
\begin{aligned}
& \mathbf{p}_{0}(e)= \begin{cases}\mathbf{q}_{1}(e) & \text { if } e \in E \backslash\left\{v_{0} v_{1}, v_{1} v_{2}\right\} \\
L_{0} & \text { if } e=v_{0} v_{1} \\
\mathbf{q}_{1}\left(v_{0} v_{2}\right) & \text { if } e=v_{1} v_{2}\end{cases} \\
& \mathbf{p}_{1}(e)= \begin{cases}\mathbf{q}_{1}(e) & \text { if } e \in E \backslash\left\{v_{0} v_{1}, v_{1} v_{2}\right\} \\
\mathbf{q}_{1}\left(v_{0} v_{2}\right) & \text { if } e=v_{0} v_{1} \\
L_{1} & \text { if } e=v_{1} v_{2}\end{cases}
\end{aligned}
$$

where $L_{0}$ is a $(d-2)$-dimensional affine subspace contained in $\Pi_{G_{1}, \mathbf{q}_{1}}\left(v_{0}\right)$ and $L_{1}$ is the one contained in $\Pi_{G_{1}, \mathbf{q}_{1}}\left(v_{2}\right)$. Then, as was shown in Claim 6.9, it is not difficult to see that $\left(G, \mathbf{p}_{0}\right)$ is a panel-hinge framework such that $\Pi_{G, \mathbf{p}_{0}}(u)=\Pi_{G_{1}, \mathbf{q}_{1}}(u)$ for $u \in$ $V \backslash\left\{v_{1}\right\}$ and $\Pi_{G, \mathbf{p}_{0}}\left(v_{1}\right)=\Pi_{G_{1}, \mathbf{q}_{1}}\left(v_{0}\right)$. Similarly, $\left(G, \mathbf{p}_{1}\right)$ is a panel-hinge framework such that $\Pi_{G, \mathbf{p}_{1}}(u)=\Pi_{G_{1}, \mathbf{q}_{1}}(u)$ for $u \in V \backslash\left\{v_{1}\right\}$ and $\Pi_{G, \mathbf{p}_{1}}\left(v_{1}\right)=\Pi_{G_{1}, \mathbf{q}_{1}}\left(v_{2}\right)$.

Let us take a look at $R\left(G, \mathbf{p}_{0}\right)$ given by

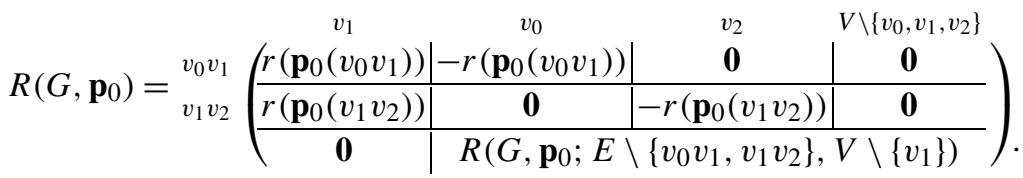


We shall apply the matrix manipulation as was given in the proof of Lemma 6.10, which converts the matrix of (6.14) to that of (6.29). Here, the vertices $v_{1}, v_{0}$ and $v_{2}$ play the roles of $v, a$ and $b$ (of $R\left(G, \mathbf{p}_{1}\right)$ in the previous lemma), respectively. The matrix $R\left(G, \mathbf{p}_{0}\right)$ of (6.49) is changed to the following form by appropriate column and row operations by using the fact that the rows associated with $v_{0} v_{2}$ in $R\left(G_{1}, \mathbf{q}_{1}\right)$ correspond to those associated with $v_{1} v_{2}$ in $R\left(G, \mathbf{p}_{0}\right)$ :

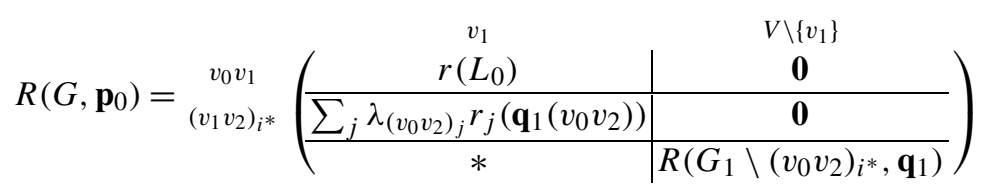

where several new notations appearing in (6.50) are defined as follows: the integer $i^{*}$ is the index of a redundant row vector among those associated with $v_{0} v_{2}$ in $R\left(G_{1}, \mathbf{q}_{1}\right)$ (such a redundant edge always exists by Claim 6.11), and $R\left(G_{1} \backslash\left\{\left(v_{0} v_{2}\right)_{i^{*}}\right\}, \mathbf{q}_{1}\right)$ denotes the matrix obtained by removing the row of $\left(v_{0} v_{2}\right)_{i}$ from $R\left(G_{1}, \mathbf{q}_{1}\right)$, which satisfies

$$
\operatorname{rank} R\left(G_{1} \backslash\left\{\left(v_{0} v_{2}\right)_{i^{*}}\right\}, \mathbf{q}_{1}\right)=\operatorname{rank} R\left(G_{1}, \mathbf{q}_{1}\right)=D(|V|-2)
$$

Also, the scalar $\lambda\left(v_{0} v_{2}\right)_{j}$ comes from the redundancy of $R\left(G_{1}, \mathbf{q}_{1} ;\left(v_{0} v_{2}\right)_{i^{*}}\right)$ within $R\left(G_{1}, \mathbf{q}_{1}\right)$, i.e., since $R\left(G_{1}, \mathbf{q}_{1} ;\left(v_{0} v_{2}\right)_{i^{*}}\right)$ is redundant in $R\left(G_{1}, \mathbf{q}_{1}\right)$, it can be expressed by a linear combination of the other row vectors of $R\left(G_{1}, \mathbf{q}_{1}\right)$ and hence we have introduced $\lambda_{e_{j}}$ for each $e \in E \backslash\left\{v_{0} v_{1}, v_{1} v_{2}\right\} \cup\left\{v_{0} v_{2}\right\}$ and $1 \leq j \leq D-1$ such that $\lambda_{\left(v_{0} v_{2}\right)_{i *}}=1$ and

$$
\sum_{\substack{e \in E \backslash\left\{v_{0} v_{1}, v_{1} v_{2}\right\} \cup\left\{v_{0} v_{2}\right\} \\ 1 \leq j \leq D-1}} \lambda_{e_{j}} R\left(G_{1}, \mathbf{q}_{1} ; e_{j}\right)=\mathbf{0} .
$$

This dependency will play a key role in the proof.

Symmetrically, we can convert $R\left(G, \mathbf{p}_{1}\right)$ to the following matrix by appropriate row and column fundamental operations:

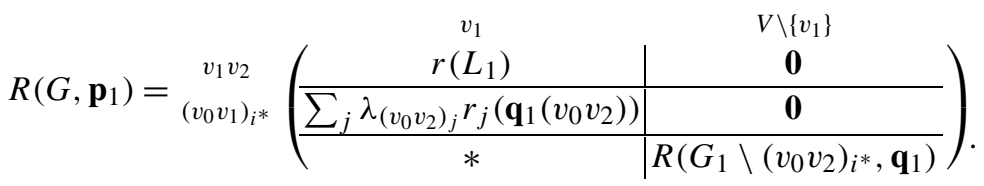

Notice that the row vectors associated with $v_{0} v_{2}$ in $R\left(G_{1}, \mathbf{q}_{1}\right)$ correspond to those associated with $v_{0} v_{1}$ in $R\left(G, \mathbf{p}_{1}\right)$.

We are now going to construct the other $d-2$ frameworks. Consider $G_{i}=$ $G_{v_{i}}^{v_{i-1} v_{i+1}}=\left(V \backslash\left\{v_{i}\right\}, E \backslash\left\{v_{i-1} v_{i}, v_{i} v_{i+1}\right\} \cup\left\{v_{i-1} v_{i+1}\right\}\right)$ for $2 \leq i \leq d-1$. We shall focus on the following isomorphism $\rho_{i}: V \backslash\left\{v_{i}\right\} \rightarrow V \backslash\left\{v_{1}\right\}$ between $G_{1}$ and $G_{i}$ for 
each $2 \leq i \leq d-1$ :

$$
\rho_{i}(u)= \begin{cases}u & \text { if } u \in V \backslash\left\{v_{1}, v_{2}, \ldots, v_{i-1}, v_{i}\right\} \\ v_{j+1} & \text { if } u=v_{j} \in\left\{v_{1}, v_{2}, \ldots, v_{i-1}\right\}\end{cases}
$$

Based on the isomorphism $\rho_{i}$, we consider the nonparallel panel-hinge framework $\left(G_{i}, \mathbf{q}_{i}\right)$ for $2 \leq i \leq d-1$, which is exactly the same framework as $\left(G_{1}, \mathbf{q}_{1}\right)$, such that

$$
\Pi_{G_{i}, \mathbf{q}_{i}}(u)=\Pi_{G_{1}, \mathbf{q}_{1}}\left(\rho_{i}(u)\right) \text { for each } u \in V \backslash\left\{v_{i}\right\} .
$$

More formally, $\left(G_{i}, \mathbf{q}_{i}\right)$ is defined by the mapping $\mathbf{q}_{i}$ on the edge set of $G_{i}$, which is $E \backslash\left\{v_{i-1} v_{i}, v_{i} v_{i+1}\right\} \cup\left\{v_{i-1} v_{i+1}\right\}$, defined as follows:

$$
\mathbf{q}_{i}(u w)=\mathbf{q}_{1}\left(\rho_{i}(u) \rho_{i}(w)\right)= \begin{cases}\mathbf{q}_{1}(u w) & \text { if } u w \in E \backslash\left\{v_{0} v_{1}, v_{1} v_{2}, \ldots, v_{d-1} v_{d}\right\} \\ \mathbf{q}_{1}\left(v_{0} v_{2}\right) & \text { if } u w=v_{0} v_{1} \\ \mathbf{q}_{1}\left(v_{j} v_{j+1}\right) & \text { if } u w=v_{j-1} v_{j} \text { for } 2 \leq j \leq i-1 \\ \mathbf{q}_{1}\left(v_{i} v_{i+1}\right) & \text { if } u w=v_{i-1} v_{i+1} \\ \mathbf{q}_{1}\left(v_{j} v_{j+1}\right) & \text { if } u w=v_{j} v_{j+1} \text { for } i+1 \leq j \leq d-1 .\end{cases}
$$

Based on $\left(G_{i}, \mathbf{q}_{i}\right)$, we shall construct the framework $\left(G, \mathbf{p}_{i}\right)$ for each $2 \leq i \leq d-1$ as follows (see Fig. 8):

$$
\mathbf{p}_{i}(e)= \begin{cases}\mathbf{q}_{i}(e) & \text { if } e \in E \backslash\left\{v_{i-1} v_{i}, v_{i} v_{i+1}\right\} \\ \mathbf{q}_{i}\left(v_{i-1} v_{i+1}\right) & \text { if } e=v_{i-1} v_{i} \\ L_{i} & \text { if } e=v_{i} v_{i+1}\end{cases}
$$

where $L_{i}$ is a $(d-2)$-dimensional affine subspace contained in $\Pi_{G_{i}, \mathbf{q}_{i}}\left(v_{i+1}\right)$. Note that $\Pi_{G_{i}, \mathbf{q}_{i}}\left(v_{i+1}\right)=\Pi_{G_{1}, \mathbf{q}_{1}}\left(\rho_{i}\left(v_{i+1}\right)\right)=\Pi_{G_{1}, \mathbf{q}_{1}}\left(v_{i+1}\right)$ by (6.54) and (6.55). In other words, $L_{i}$ is taken as a $(d-2)$-dimensional affine subspace satisfying

$$
L_{i} \subset \Pi_{G_{1}, \mathbf{q}_{1}}\left(v_{i+1}\right)
$$

As was shown in Claim 6.9, it is not difficult to see that $\left(G, \mathbf{p}_{i}\right)$ is a panel-hinge framework satisfying $\Pi_{G, \mathbf{p}_{i}}(u)=\Pi_{G_{i}, \mathbf{q}_{i}}(u)=\Pi_{G_{1}, \mathbf{q}_{1}}\left(\rho_{i}(u)\right)$ for each $u \in V \backslash\left\{v_{i}\right\}$ and $\Pi_{G, \mathbf{p}_{i}}\left(v_{i}\right)=\Pi_{G_{i}, \mathbf{q}_{i}}\left(v_{i+1}\right)=\Pi_{G_{1}, \mathbf{q}_{1}}\left(v_{i+1}\right)$. Hence, $\left(G, \mathbf{p}_{i}\right)$ is a panel-hinge framework such that only the panels of $v_{i}$ and $v_{i+1}$ coincide and all the other pairs of panels are nonparallel. We remark that $\left(G, \mathbf{p}_{i}\right)$ can be converted to a nonparallel panel-hinge framework without decreasing the rank of the rigidity matrix by Lemma 5.2.

Combining (6.56) and (6.57), we have, for $2 \leq i \leq d-1$,

$$
\begin{aligned}
\mathbf{p}_{i}(e) & =\mathbf{q}_{1}(e) & & \text { for } e \in E \backslash\left\{v_{0} v_{1}, v_{1} v_{2}, \ldots, v_{d-1} v_{d}\right\} \\
\mathbf{p}_{i}\left(v_{0} v_{1}\right) & =\mathbf{q}_{1}\left(v_{0} v_{2}\right) & & \\
\mathbf{p}_{i}\left(v_{j-1} v_{j}\right) & =\mathbf{q}_{1}\left(v_{j} v_{j+1}\right) & & \text { for } 2 \leq j \leq i \\
\mathbf{p}_{i}\left(v_{i} v_{i+1}\right) & =L_{i} & & \\
\mathbf{p}_{i}\left(v_{j} v_{j+1}\right) & =\mathbf{q}_{1}\left(v_{j} v_{j+1}\right) & & \text { for } i+1 \leq j \leq d-1 \text { (if } i \neq d-1) .
\end{aligned}
$$




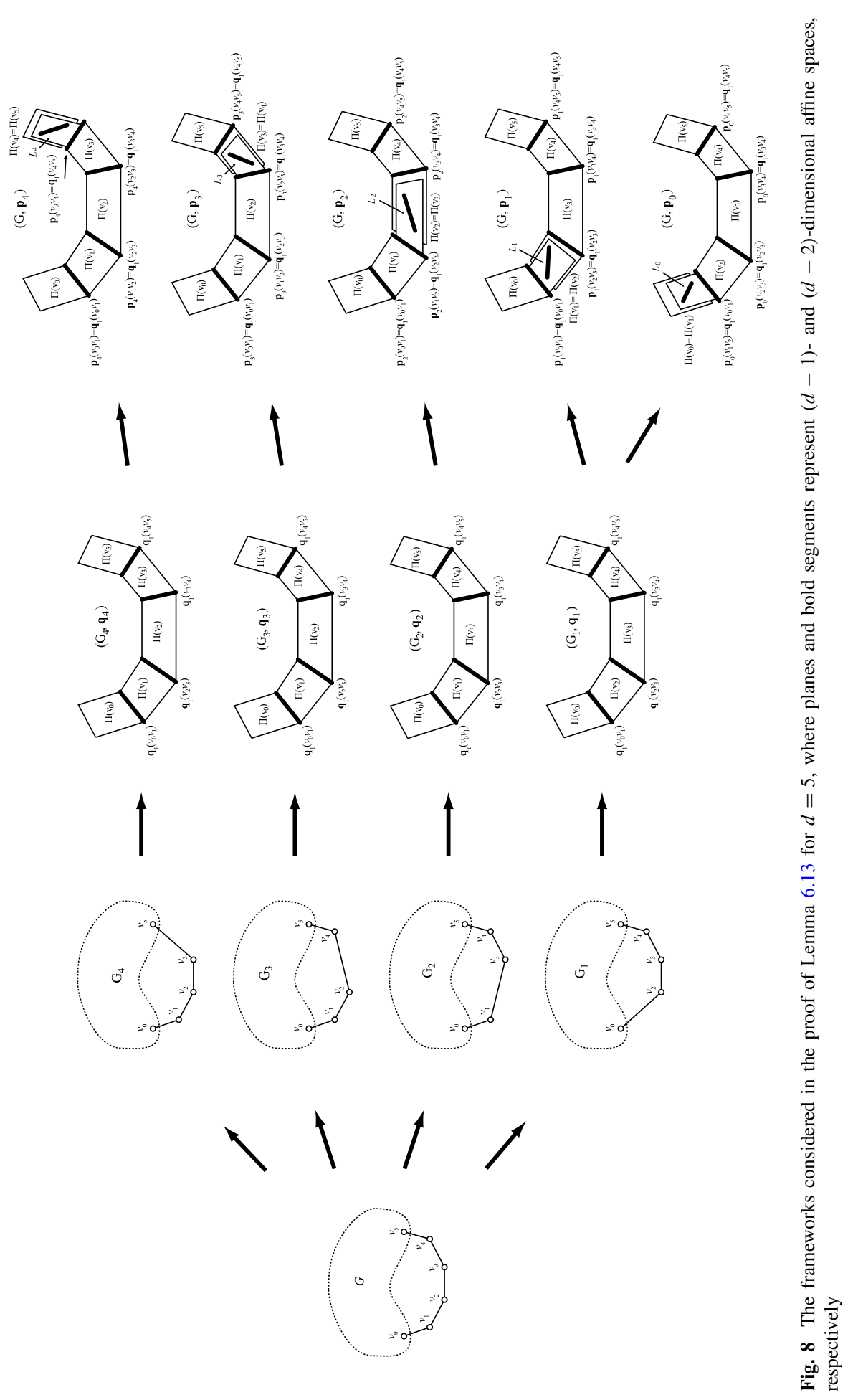


Let us consider $R\left(G, \mathbf{p}_{i}\right)$ :

$$
R\left(G, \mathbf{p}_{i}\right)={ }_{v_{i} v_{i+1}} v_{i-1} v_{i}\left(\begin{array}{c|c|c|c}
v_{i} & v_{i+1} & v_{i-1} & \multicolumn{2}{c}{\begin{array}{c}
V \backslash\left\{v_{i-1}, v_{i}, v_{i+1}\right\} \\
\left.\left(v_{i} v_{i+1}\right)\right)
\end{array}} & -r\left(\mathbf{p}_{i}\left(v_{i} v_{i+1}\right)\right) & \mathbf{0} & \mathbf{0} \\
\hline r\left(\mathbf{p}_{i}\left(v_{i-1} v_{i}\right)\right) & \mathbf{0} & -r\left(\mathbf{p}_{i}\left(v_{i-1} v_{i}\right)\right) & \mathbf{0} \\
\hline \mathbf{0} & R\left(G, \mathbf{p}_{i} ; E \backslash\left\{v_{i-1} v_{i}, v_{i} v_{i+1}\right\}, V \backslash\left\{v_{i}\right\}\right)
\end{array}\right) .
$$

We now convert $R\left(G, \mathbf{p}_{i}\right)$ to the matrix which contains $R\left(G_{1}, \mathbf{q}_{1}\right)$ as its submatrix; perform the column operations which add the $j$ th column of $R\left(G, \mathbf{p}_{i} ; v_{i}\right)$ to that of $R\left(G, \mathbf{p}_{i} ; v_{i+1}\right)$ for each $1 \leq j \leq D$ and then substitute all of (6.59) into the resulting matrix. Then, it is not difficult to see that $R\left(G, \mathbf{p}_{i}\right)$ of (6.60) is changed to

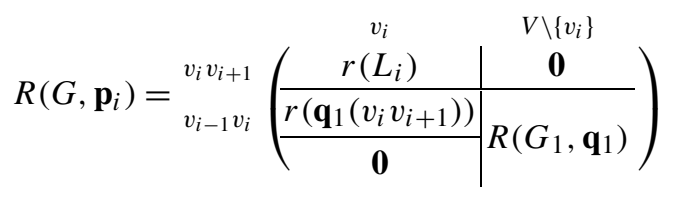

where we used the following row correspondence between $R\left(G, \mathbf{p}_{i} ; E \backslash\left\{v_{i} v_{i+1}\right\}\right.$, $\left.V \backslash\left\{v_{i}\right\}\right)$ and $R\left(G_{1}, \mathbf{q}_{1}\right)$ derived from (6.59):

$$
\begin{aligned}
& \left.\begin{array}{l}
R\left(G, \mathbf{p}_{i}\right) \\
e \\
v_{0} v_{1} \\
v_{j-1} v_{j} \\
v_{j^{\prime}} v_{j^{\prime}+1}
\end{array}\right\} \Longleftrightarrow \begin{cases}e & \text { for } e \in E \backslash\left\{v_{0} v_{1}, \ldots, v_{d-1} v_{d}\right\} \\
v_{0} v_{2} & \\
v_{j} v_{j+1} & \text { for } 2 \leq j \leq i \\
v_{j^{\prime}} v_{j^{\prime}+1} & \text { for } i+1 \leq j^{\prime} \leq d-1\end{cases}
\end{aligned}
$$

(and the column correspondence follows from the isomorphism $\rho_{i}$ defined in (6.54)). In particular, the row associated with $\left(v_{0} v_{2}\right)_{i *}$ in $R\left(G_{1}, \mathbf{q}_{1}\right)$ corresponds to the row associated with $\left(v_{0} v_{1}\right)_{i *}$ in $R\left(G, \mathbf{p}_{i}\right)$.

Recall that the row of $R\left(G_{1}, \mathbf{q}_{1}\right)$ associated with $\left(v_{0} v_{2}\right)_{i^{*}}$ is redundant. As before, we consider the row operations which convert $R\left(G_{1}, \mathbf{q}_{1} ;\left(v_{0} v_{2}\right)_{i^{*}}\right)$ to a zero vector and apply these row operations to the matrix $R\left(G, \mathbf{p}_{i}\right)$ of (6.61). More precisely, following the row correspondence described in (6.62), we shall perform the fundamental row operations which add the row vectors of $R\left(G, \mathbf{p}_{i} ; E \backslash\left\{v_{i} v_{i+1}\right\}\right)$ to the row $R\left(G, \mathbf{p}_{i} ;\left(v_{0} v_{1}\right)_{i}{ }^{*}\right)$ with the weight $\lambda_{e_{j}}$. Namely, the row operations change the row associated with $\left(v_{0} v_{1}\right)_{i *}$ in (6.61) to the following row vector:

$$
\begin{aligned}
& \sum_{1 \leq j \leq D-1} \lambda_{\left(v_{0} v_{2}\right)_{j}} R\left(G, \mathbf{p}_{i} ;\left(v_{0} v_{1}\right)_{j}\right) \\
& +\sum_{2 \leq j^{\prime} \leq i, 1 \leq j \leq D-1} \lambda_{\left(v_{j^{\prime}} v_{j^{\prime}+1}\right)_{j}} R\left(G, \mathbf{p}_{i} ;\left(v_{j^{\prime}-1} v_{j^{\prime}}\right)_{j}\right)
\end{aligned}
$$




$$
\begin{aligned}
& +\sum_{\substack{i+1 \leq j^{\prime} \leq d-1 \\
1 \leq j \leq D-1}} \lambda_{\left(v_{j^{\prime}} v_{j^{\prime}+1}\right)_{j}} R\left(G, \mathbf{p}_{i} ;\left(v_{j^{\prime}} v_{j^{\prime}+1}\right)_{j}\right) \\
& +\sum_{\substack{e \in E \backslash\left\{v_{0} v_{1}, \ldots, v_{d-1} v_{d}\right\} \\
1 \leq j \leq D-1}} \lambda_{e_{j}} R\left(G, \mathbf{p}_{i} ; e_{j}\right)
\end{aligned}
$$

where $\lambda\left(v_{0} v_{2}\right)_{i *}=1$.

By (6.52), all the entries of the part of the new row vector (6.63) associated with $V \backslash\left\{v_{i}\right\}$ become zero. Also, as for the rest part of the new row (6.63) associated with $v_{i}$, since the entries of $R\left(G, \mathbf{p}_{i} ; E \backslash\left\{v_{i-1} v_{i}, v_{i} v_{i+1}\right\}, v_{i}\right)$ of (6.61) are all zero, the row operations change $R\left(G, \mathbf{p}_{i} ;\left(v_{0} v_{1}\right)_{i^{*}}, v_{i}\right)$ to

$$
\sum_{1 \leq j \leq D-1} \lambda\left(v_{i} v_{i+1}\right)_{j} r_{j}\left(\mathbf{p}_{i}\left(v_{i-1} v_{i}\right)\right)
$$

which is equal to

$$
\sum_{1 \leq j \leq D-1} \lambda_{\left(v_{i} v_{i+1}\right)_{j}} r_{j}\left(\mathbf{q}_{1}\left(v_{i} v_{i+1}\right)\right)
$$

since $\mathbf{p}_{i}\left(v_{i-1} v_{i}\right)=\mathbf{q}_{1}\left(v_{i} v_{i+1}\right)$ by (6.59). Therefore, the fundamental row operations actually change $R\left(G, \mathbf{p}_{i}\right)$ of (6.61) to the following matrix (up to some row exchange of $\left.\left(v_{0} v_{1}\right)_{i}\right)$ :

$$
R\left(G, \mathbf{p}_{i}\right)=\begin{array}{c|c}
v_{i} & V \backslash\left\{v_{i}\right\} \\
v_{i} v_{i+1} \\
\left(v_{0} v_{1}\right)_{i^{*}}
\end{array}\left(\begin{array}{c|c}
r\left(L_{i}\right) & \mathbf{0} \\
\hline \sum_{j} \lambda\left(v_{i} v_{i+1}\right)_{j} r_{j}\left(\mathbf{q}_{1}\left(v_{i} v_{i+1}\right)\right) & \mathbf{0} \\
\hline * & R\left(G_{1} \backslash\left(v_{0} v_{2}\right)_{i^{*}}, \mathbf{q}_{1}\right)
\end{array}\right) .
$$

Let us denote the top-left $D \times D$-submatrices of (6.50) and (6.53) by $M_{0}$ and $M_{1}$, and also that of (6.64) by $M_{i}$ for $2 \leq i \leq d-1$, i.e.,

$$
\begin{aligned}
& M_{0}=\left(\begin{array}{c}
r\left(L_{0}\right) \\
\sum_{j} \lambda_{\left(v_{0} v_{2}\right)_{j}} r_{j}\left(\mathbf{q}\left(v_{0} v_{2}\right)\right)
\end{array}\right) \quad M_{1}=\left(\begin{array}{c}
r\left(L_{1}\right) \\
\sum_{j} \lambda_{\left(v_{0} v_{2}\right)_{j}} r_{j}\left(\mathbf{q}\left(v_{0} v_{2}\right)\right)
\end{array}\right) \\
& M_{i}=\left(\begin{array}{c}
r\left(L_{i}\right) \\
\sum_{j} \lambda_{\left(v_{i} v_{i+1}\right)_{j}} r_{j}\left(\mathbf{q}\left(v_{i} v_{i+1}\right)\right)
\end{array}\right) \quad \text { for } 2 \leq i \leq d-1 .
\end{aligned}
$$

Recall that $L_{0}$ can be taken as any $(d-2)$-dimensional affine subspace satisfying $L_{0} \subset \Pi_{G_{1}, \mathbf{q}_{1}}\left(v_{0}\right)$, while, for $1 \leq i \leq d-1, L_{i}$ can be any $(d-2)$-dimensional affine subspace satisfying $L_{i} \subset \Pi_{G_{1}, \mathbf{q}_{1}}\left(v_{i+1}\right)$. Then, as in the proof of 3-dimensional case, the remaining task is to show the following fact:

At least one of $M_{0}, M_{1}, \ldots, M_{d-1}$ has full rank for an $L_{i}, 0 \leq i \leq d-1$.

If this is true, then we obtain $\operatorname{rank} R\left(G, \mathbf{p}_{i}\right) \geq \operatorname{rank} M_{i}+\operatorname{rank} R\left(G_{1} \backslash\left\{\left(v_{0} v_{2}\right)_{i}{ }^{*}\right\}, \mathbf{q}_{1}\right)=$ $D+D(|V|-2)=D(|V|-1)$ by $(6.51)$. 
Let us show (6.65). Let $r=\sum_{j} \lambda_{\left(v_{0} v_{2}\right)_{j}} r_{j}\left(\mathbf{q}\left(v_{0} v_{2}\right)\right) \in \mathbb{R}^{D}$. Suppose $M_{0}$ (and $M_{1}$, resp.) does not have full rank. Then, $r$ is contained in the row space of $r\left(L_{0}\right)$ (and $r\left(L_{1}\right)$, resp.), that is, $r$ is contained in the orthogonal complement of the vector space spanned by a $(d-1)$-extensor $C\left(L_{0}\right)$ (and that of $C\left(L_{1}\right)$, respectively). Also, due to the fact that $v_{i}$ is a vertex of degree two in $G_{1}$ for all $2 \leq i \leq d-1$, we can easily show the following fact in a manner similar to the previous lemma (cf. (6.44)):

$$
\sum_{1 \leq j \leq D-1} \lambda\left(v_{i} v_{i+1}\right)_{j} r_{j}\left(\mathbf{q}\left(v_{i} v_{i+1}\right)\right)= \pm r \quad \text { for } 2 \leq i \leq d-1 .
$$

Notice that (6.66) implies that $M_{i}$ does not have full rank if and only if $r$ is contained in the orthogonal complement of the vector space spanned by $C\left(L_{i}\right)$. Therefore, none of $M_{0}, M_{1}, \ldots, M_{d-1}$ has full rank for any choice of $L_{i}, 0 \leq i \leq d-1$ if and only if $r$ is contained in the orthogonal complement of the vector space spanned by

$$
\bigcup_{0 \leq i \leq d-1}\left(\bigcup_{L_{i} \subset \Pi_{i}} C\left(L_{i}\right)\right)
$$

where $\Pi_{0}=\Pi_{G_{1}, \mathbf{q}_{1}}\left(v_{0}\right)$ and $\Pi_{i}=\Pi_{G_{1}, \mathbf{q}_{1}}\left(v_{i+1}\right)$ for each $i$ with $1 \leq i \leq d-1$. Therefore, in order to show that at least one of $M_{0}, M_{1}, \ldots, M_{d-1}$ has full rank, it is enough to show that the dimension of the vector space spanned by (6.67) is equal to $D$.

Since the framework $\left(G_{1}, \mathbf{q}_{1}\right)$ is a generic nonparallel framework, the set of the coefficients appearing in the equations expressing $\Pi_{i}, 0 \leq i \leq d-1$ is algebraically independent over the rational field. Therefore, for any $j$ hyperplanes among them, their intersection forms a $(d-j)$-dimensional affine space. We take $d+1$ distinct points in $\mathbb{R}^{d}$ as follows: for each $0 \leq i \leq d-1, p_{i} \in \bigcap_{0 \leq j \leq d-1, j \neq i} \Pi_{j} \backslash \Pi_{i}$ and $p_{d}=$ $\bigcap_{0 \leq j \leq d-1} \Pi_{j}$. Clearly, $\left\{p_{0}, p_{1}, \ldots, p_{d}\right\}$ is affinely independent, and also notice that any $(d-2)$-dimensional affine subspace spanned by $d-1$ points among them is contained in $\bigcup_{0 \leq j \leq d-1} \Pi_{j}$. This implies that any $(d-1)$-extensor obtained from $d-1$ points belongs to the set of (6.67). Thus the dimension of the vector space spanned by (6.67) is equal to $\left(\begin{array}{l}d+1 \\ d-1\end{array}\right)=D$ by Lemma 2.1 .

This completes the proof of Lemma 6.13 as well as Theorem 5.5 in general dimension.

\section{Conclusion}

We have proved that any body-hinge rigid graph can be realized as an infinitesimally rigid panel-hinge framework based on the new inductive sequence of minimally body-hinge rigid graphs. This settles the Molecular Conjecture affirmatively for general dimension. Also, as a corollary we found that a 3-dimensional bar-joint framework $\left(G^{2}, \mathbf{p}\right)$ is infinitesimally rigid on a generic joint configuration if and only if $5 G$ contains six edge-disjoint spanning trees. This provides the mathematical validity of using the pebble game algorithms for computing the degree of freedom of molecules.

We should remark that the study of a bar-joint realization of the square of a graph has not been completed yet. Indeed, Corollary 5.7 tells us how to compute the rank 
of the whole graph only, and the explicit formula of the rank function has not been clarified yet. The research should be continued to understand general 3-dimensional bar-joint frameworks whose combinatorial characterization remains unexplained.

Acknowledgements We would like to thank Audrey Lee-St. John, Meera Sitharam, Ileana Streinu and Louis Theran for giving several useful comments on our draft paper at Barbados Workshop 2009: Geometric constraints with applications in CAD and biology. Also, we would like to thank Shuji Kijima for giving a lot of comments on the draft version.

The first author is supported by Grant-in-Aid for Scientific Research (B) and Grant-in-Aid for Scientific Research (C), JSPS. The second author is supported by Grant-in-Aid for JSPS Research Fellowships for Young Scientists.

\section{References}

1. Amato, N.M.: The Protein Floding Server. Parasol, Texas A\&M University, http://parasol.tamu.edu/ groups/amatogroup/

2. Barnabei, M., Brini, A., Rota, G.: On the exterior calculus of invariant theory. J. Algebra 96(1), 120160 (1985)

3. Catlin, P., Grossman, J., Hobbs, A., Lai, H.-J.: Fractional arboricity, strength, and principal partitions in graphs and matroids. Discrete Appl. Math. 40(3), 285-302 (1992)

4. Crapo, H., Whiteley, W.: Statics of frameworks and motions of panel structures: a projective geometric introduction. Struct. Topol. 6, 43-82 (1982)

5. Cunningham, W.: Optimal attack and reinforcement of a network. J. ACM 32(3), 549-561 (1985)

6. Doubilet, P., Rota, G.-C., Stein, J.: On the foundations of combinatorial theory IX. Combinatorial methods in invariant theory. Stud. Appl. Math. 53(3), 185-216 (1974)

7. Flexweb Server: http://flexweb.asu.edu

8. Graver, J.E., Servatius, B., Servatius, H.: Combinatorial Rigidity. Graduate Studies in Mathematics, vol. 2, p. 11. American Mathematical Society, New York (1993)

9. Gusfield, D.: Connectivity and edge-disjoint spanning trees. Inf. Process. Lett. 16(2), 87-89 (1983)

10. Jackson, B., Jordán, T.: Rank and independence in the rigidity matroid of molecular graphs. Technical Report TR-2006-02, Egerváry Research Group (2006)

11. Jackson, B., Jordán, T.: Rigid components in molecular graphs. Algorithmica 48(4), 399-412 (2007)

12. Jackson, B., Jordán, T.: Brick partitions of graphs. Discrete Math. 310(2), 270-275 (2008)

13. Jackson, B., Jordán, T.: On the rigidity of molecular graphs. Combinatorica 28(6), 645-658 (2008)

14. Jackson, B., Jordán, T.: Pin-collinear body-and-pin frameworks and the molecular conjecture. Discrete Comput. Geom. 40(2), 258-278 (2008)

15. Jackson, B., Jordán, T.: The generic rank of body-bar-and-hinge frameworks. Eur. J. Comb. 31(2), 574-588 (2009)

16. Jacobs, D.J., Kuhn, L.A., Thorpe, M.F.: Flexible and rigid regions in proteins. In: Rigidity Theory and Applications, pp. 357-384. Kluwer, Amsterdam (1999)

17. Lee, A., Streinu, I.: Pebble game algorithms and sparse graphs. Discrete Math. 308(8), 1425-1437 (2008)

18. Lei, M., Zavodszky, M., Kuhn, L., Thorpe, M.: Sampling protein conformations and pathways. J. Comput. Chem. 25(9), 1133-1148 (2004)

19. Lovász, L.: Combinatorial Problems and Exercises, 2nd edn. American Mathematical Society, New York (2007)

20. Nash-Williams, C.: Edge-disjoint spanning trees of finite graphs. J. Lond. Math. Soc. 1(1), 445-450 (1961)

21. Oxley, J.: Matroid Theory. Oxford University Press, Oxford (1992)

22. Schrijver, A.: Combinatorial Optimization: Polyhedra and Efficiency. Springer, Berlin (2003)

23. Selig, J.: Geometric Fundamentals of Robotics, 2nd edn. Springer, Berlin (2004)

24. Tay, T.: Linking $(n-2)$-dimensional panels in $n$-space II: $(n-2,2)$-frameworks and body and hinge structures. Graphs Comb. 5(1), 245-273 (1989)

25. Tay, T.S., Whiteley, W.: Recent advances in the generic rigidity of structures. Struct. Topol. 9, 31-38 (1984) 
26. Tutte, W.T.: On the problem of decomposing a graph into $n$ connected factors. J. Lond. Math. Soc. 36, 221-230 (1961)

27. Wells, S., Menor, S., Hespenheide, B., Thorpe, M.F.: Constrained geometric simulation of diffusive motion in proteins. Phys. Biol. 2, S127-S136 (2005)

28. White, N.: Grassmann-Cayley algebra and robotics. J. Intell. Robot. Syst. 11, 91-107 (1994)

29. White, N., Whiteley, W.: The algebraic geometry of motions of bar-and-body frameworks. SIAM J. Algebr. Discrete Methods 8(1), 1-32 (1987)

30. Whiteley, W.: Rigidity and polarity I: Statics of sheet structures. Geom. Dedic. 22, 329-362 (1987)

31. Whiteley, W.: The union of matroids and the rigidity of frameworks. SIAM J. Discrete Math. 1(2), 237-255 (1988)

32. Whiteley, W.: A matroid on hypergraphs with applications in scene analysis and geometry. SIAM J. Discrete Math. 4, 75-95 (1989)

33. Whiteley, W.: Some matroids from discrete applied geometry. Contemp. Math. 197, 171-312 (1996)

34. Whiteley, W.: Rigidity of molecular structures: geometric and generic analysis. In: Thorpe, O., Duxbury, O. (eds.) Rigidity Theory and Applications, pp. 21-46. Kluwer, Amsterdam (1999)

35. Whiteley, W.: The equivalence of molecular rigidity models as geometric and generic graphs. Preprint (2004)

36. Whiteley, W.: Rigidity and scene analysis. In: Goodman, J.E., O'Rourke, J. (eds.) Handbook of Discrete and Computational Geometry, 2nd edn. pp. 1327-1354. CRC Press, Boca Raton (2004), Chap. 60

37. Whiteley, W.: Counting out to the flexibility of molecules. Phys. Biol. 2, S116-S126 (2005) 